\title{
Holographic entanglement entropy for perturbative higher-curvature gravities
}

\author{
Pablo Bueno, ${ }^{a}$ Joan Camps ${ }^{b}$ and Alejandro Vilar López ${ }^{c, d}$ \\ ${ }^{a}$ Instituto Balseiro, Centro Atómico Bariloche, \\ 8400-S.C. de Bariloche, Río Negro, Argentina \\ ${ }^{b}$ Department of Physics and Astronomy, University College London, \\ Gower Street, London WC1E 6BT, United Kingdom \\ ${ }^{c}$ Departamento de Física de Partículas, Universidade de Santiago de Compostela, \\ E-15782 Santiago de Compostela, Spain \\ ${ }^{d}$ Instituto Galego de Física de Altas Enerxías (IGFAE), Universidade de Santiago de Compostela, \\ E-15782 Santiago de Compostela, Spain \\ E-mail: pablo.bueno@cab.cnea.gov.ar, joan.camps@gmail.com, \\ alejandrovilar.lopez@usc.es
}

ABSTRACT: The holographic entanglement entropy functional for higher-curvature gravities involves a weighted sum whose evaluation, beyond quadratic order, requires a complicated theory-dependent splitting of the Riemann tensor components. Using the splittings of general relativity one can obtain unambiguous formulas perturbatively valid for general higher-curvature gravities. Within this setup, we perform a novel rewriting of the functional which gets rid of the weighted sum. The formula is particularly neat for general cubic and quartic theories, and we use it to explicitly evaluate the corresponding functionals. In the case of Lovelock theories, we find that the anomaly term can be written in terms of the exponential of a differential operator. We also show that order- $n$ densities involving $n_{R}$ Riemann tensors (combined with $n-n_{R}$ Ricci's) give rise to terms with up to $2 n_{R}-2$ extrinsic curvatures. In particular, densities built from arbitrary Ricci curvatures combined with zero or one Riemann tensors have no anomaly term in their functionals. Finally, we apply our results for cubic gravities to the evaluation of universal terms coming from various symmetric regions in general dimensions. In particular, we show that the universal function characteristic of corner regions in $d=3$ gets modified in its functional dependence on the opening angle with respect to the Einstein gravity result.

Keywords: AdS-CFT Correspondence, Conformal Field Theory, Classical Theories of Gravity

ARXIV EPRINT: 2012.14033 


\section{Contents}

1 Introduction 1

1.1 Notation and conventions 3

2 GR splittings for perturbative higher-curvature theories 5

3 Rewriting the HEE functional $\quad 7$

$\begin{array}{lll}3.1 & \text { Symmetry factors in derivatives and some notation } & 7\end{array}$

3.2 New form of the HEE functional 9

$\begin{array}{ll}\text { 3.3 Anomaly term in Lovelock theories } & 14\end{array}$

3.4 Anomaly term for cubic gravities 16

3.5 Anomaly term for quartic gravities 16

$\begin{array}{lll}3.6 & \text { An example mixing type } A \text { and type } B \text { terms } & 17\end{array}$

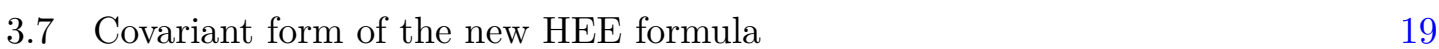

4 Explicit covariant form of the functionals $\quad 21$

$4.1 f(R)$ gravities 21

4.2 Lovelock gravities 21

4.3 Quadratic gravities 22

4.4 Cubic gravities 23

4.5 Quartic gravities 25

$4.6 \mathcal{L}\left(g_{\mu \nu}, R_{\rho \sigma}\right)$ gravities 31

4.7 General structure depending on the number of Riemann tensors 32

5 Universal terms 33

$\begin{array}{lll}5.1 & \text { Spherical regions } & 35\end{array}$

$\begin{array}{lll}5.2 & \text { Slab regions } & 37\end{array}$

5.3 Cylinder regions 40

5.3.1 Four dimensions 41

5.3.2 Six dimensions 43

$\begin{array}{lll}5.4 & \text { Corner regions } & 45\end{array}$

6 Final comments $\quad 54$

A Proof of identities (3.26) and (3.28) 55 


\section{Introduction}

In effective (super)gravity actions, higher-curvature terms appear as stringy and/or quantum corrections to the corresponding two-derivative actions - see e.g., [1-3]. In the AdS/CFT context [4-6], the holographic duals of such modified actions are inequivalent to the ones defined by Einstein gravity (e.g., the trace anomaly coefficients in four dimensions, $a$ and $c$, no longer coincide in general). This extends beyond explicit top-down constructions and, in fact, particular higher-curvature models - e.g., with certain special properties which makes them more appealing — can be used to probe interesting CFT physics [7-11]. In some cases, this approach has been used to unveil universal properties valid for completely general CFTs [12-21].

An important entry in the holographic dictionary corresponds to entanglement entropy (EE), which for holographic theories dual to Einstein gravity (plus possible additional matter fields) can be computed using the Ryu-Takayanagi (RT) prescription [22, 23]. According to this, the EE for a region $A$ in the boundary CFT is obtained as the area of the bulk surface, $\Gamma_{A}$, which has the smallest area amongst all bulk surfaces which are homologous to $A$, divided by $4 G$, i.e.,

$$
S_{\mathrm{HEE}}^{\mathrm{E}}(A)=\frac{\mathcal{A}\left(\Gamma_{A}\right)}{4 G},
$$

where the "E" stands for Einstein gravity. When the action includes higher-curvature terms, the area functional needs to be modified, similarly to the way the BekensteinHawking black hole entropy formula [24, 25] is replaced by Wald's one [26, 27]. The naive modification which would correspond to replacing eq. (1.1) by the same Wald functional fails for entanglement entropy [28], and additional terms involving extrinsic curvatures of the generalized bulk surface are required. A hint of this is the fact that for Lovelock gravities, the result obtained from Wald's entropy differs from the alternative JacobsonMyers functional [29] by terms of that type, which generically vanish for Killing horizons, but not for holographic entangling surfaces. The right expression for the holographic entanglement entropy (HEE) functional in the case of quadratic gravities was obtained in [30]. Building up on the generalized entropy methods of [31], a general formula (in principle) valid for theories involving arbitrary contractions of Riemann tensors and metrics was obtained in [32, 33]. Schematically, it has the form

$$
S_{\mathrm{HEE}}^{\mathcal{L}_{E}(\text { Riemann })}(A)=S_{\text {Wald }}+S_{\text {Anomaly }},
$$

where, in addition to a Wald-like piece, there appears an extra "anomaly" term involving extrinsic curvatures of the generalized holographic surface. In adapted coordinates - see subsection 1.1 below for our conventions - these two terms read ${ }^{1,2}$

$$
\begin{aligned}
S_{\text {Wald }} & =2 \pi \int_{\Gamma_{A}} \mathrm{~d}^{d-1} y \sqrt{h} \frac{\partial \mathcal{L}_{E}}{\partial R_{z \bar{z} z \bar{z}}} \\
S_{\text {Anomaly }} & =2 \pi \int_{\Gamma_{A}} \mathrm{~d}^{d-1} y \sqrt{h} \sum_{\alpha}\left(\frac{\partial^{2} \mathcal{L}_{E}}{\partial R_{z i z j} \partial R_{\bar{z} k \bar{z} l}}\right)_{\alpha} \frac{8 K_{z i j} K_{\bar{z} k l}}{\left(1+q_{\alpha}\right)} .
\end{aligned}
$$

\footnotetext{
${ }^{1}$ Interesting additional developments and explorations include [34-41].

${ }^{2}$ Generalizations of eq. (1.2) to the case in which covariant derivatives of the Riemann appear in the action have also been presented [42].
} 
In principle, the generalized holographic surface $\Gamma_{A}$ should be obtained by extremizing the new functional [43]. In the anomaly term, once the second derivative is performed, each of the Riemann tensor components appearing in the resulting expression has to be split into sums of pieces with different weights $q_{\alpha}$ according to some prescription. That prescription depends on the way the conical defect appearing near the entangling region in the replica trick approach is regulated. As observed and studied in [42, 44-46], this procedure is nonunique, which leads to the so-called "splitting problem". ${ }^{3}$ While the choice of splittings does not affect $f(R)$, Lovelock or quadratic theories, it does play a crucial rôle for general theories involving $n \geq 3$ densities. ${ }^{4}$

The right splittings could in principle be identified for each particular theory by imposing that the relevant bulk geometry satisfies the corresponding equations of motion. In doing so, one would be left with a functional ready to extremize, and the resulting on-shell evaluation would yield non-perturbative results for the HEE of the corresponding theory. Doing this in practice is a highly non-trivial task which has not been pursued for explicit higher-curvature theories so far. If one followed this approach, another relevant issue would arise. For generic higher-curvature theories, the equations of motion implementing the extremization of the functional are not second-order in derivatives, so it is not completely clear how to deal with the associated boundary value problem in those cases.

A different approach, which we follow here, entails considering holographic entangling surfaces which extremize the RT functional (1.1) along with the splittings prescribed by Einstein gravity. By doing so, we avoid the boundary-value-problem issues associated to higher-order equations, and the results obtained are perturbatively valid at leading order in the higher-curvature couplings [46]. Within this framework, we manage to get rid of the $\alpha$ sum in the anomaly piece (1.4) and obtain a general expression which can be compactly written as $^{5}$

$$
S_{\text {Anomaly }}=32 \pi \int_{\Gamma_{A}} \mathrm{~d}^{d-1} y \sqrt{h}\left[\int_{0}^{1} \mathrm{~d} u u \mathrm{e}^{-F(u)}\left(\frac{\partial^{2} \mathcal{L}_{E}}{\partial R_{z i z j} \partial R_{\bar{z} k \bar{z} l}} K_{z i j} K_{\bar{z} k l}\right)\right],
$$

where the operator appearing in the exponential takes the form ${ }^{6}$

$$
F(u) \equiv\left[\left(1-u^{2}\right) \mathcal{K}_{A I} \hat{\partial}^{A I}+(1-u) \mathcal{K}_{B J} \hat{\partial}^{B J}\right]
$$

and where $\mathcal{K}_{A I} \hat{\partial}^{A I}$ and $\mathcal{K}_{B I} \hat{\partial}^{B I}$ are differential operators involving derivatives with respect to particular Riemann tensor components contracted with extrinsic and Riemann curvature components. They appear defined in eq. (3.31) and eq. (3.32) respectively. This new form of the functional becomes particularly simple for cubic and quartic theories - see

\footnotetext{
${ }^{3}$ See subsection 2 for a more detailed summary of the discussion included in this paragraph and the following two.

${ }^{4}$ The final form of the anomaly term once the (some) splitting procedure and the sum over $\alpha$ are performed differs considerably from eq. (1.4). In particular, for $n$-order densities, it may contain terms involving up to $2(n-1)$ extrinsic curvatures. This is evident from our new expression in eq. (1.5).

${ }^{5}$ See section 3.7 for the covariant form.

${ }^{6}$ As explained later, there is a normal ordering prescription implicit in this expression which forces derivatives to act exclusively on the object in the parentheses - see eq. (3.18) below.
} 
eq. (3.46) and eq. (3.48) respectively - and we use it to evaluate the explicit (covariant) HEE functionals for all cubic and quartic densities. The result for Lovelock theories is also rather suggestive - see eq. (3.40). Using our new results, we are also able to show that densities constructed exclusively from Ricci curvatures have a vanishing anomaly term, similarly to the well-known case of $f(R)$ gravities. This also extends to densities involving a single Riemann tensor contracted with Ricci curvatures. More generally, we prove that an order- $n$ density involving $n_{R}$ Riemann curvatures and $n-n_{R}$ Ricci curvatures can produce HEE functionals containing at most $2\left(n_{R}-1\right)$ extrinsic curvatures. As an application of our results, we compute a variety of universal contributions to the EE coming from various symmetric regions in general dimensions for holographic theories dual to cubic gravities. Particularly interesting are the results for strips, for which no alternative interpretation of their coefficients exists beyond EE, and for corners, for which the functional form of the Einstein gravity function only starts to get modified at cubic order.

The remainder of the paper goes as follows. In subsection 1.1 we introduce our conventions and some notation. In section 2 we briefly review the construction that leads to the general form of the holographic entanglement entropy functional, the issue with the Riemann tensor splittings and the choice that allows us to obtain results perturbatively valid for general higher-curvature theories. In section 3 we derive a new formula for the anomaly piece of the HEE functional valid for perturbative higher-curvature corrections to Einstein gravity. We show how the formula gets considerably simplified in the cases of cubic, quartic and Lovelock densities. We also illustrate how our formula should be used in concrete cases by performing a detailed example for a term coming from quintic densities, verifying the match with the $\alpha$-expansion method. In section 4 we present the explicit form of the HEE functionals for general: $f(R)$, Lovelock, quadratic, cubic, quartic, $\mathcal{L}$ (Ricci) and $R_{\mu \nu \rho \sigma} T^{\mu \nu \rho \sigma}$ (Ricci) densities in covariant form. We also prove here that the functionals corresponding to densities involving $n-n_{R}$ Ricci tensors contain at most $2\left(n_{R}-1\right)$ extrinsic curvatures. In section 5 we evaluate, for general quadratic and cubic theories, the universal entanglement entropy coefficients characterizing spheres and strips in general dimensions, cylinders in $d=4$ and $d=6$ and corners in $d=3$. For the latter, we show that the functional dependence on the opening angle of the corner gets modified by the introduction of cubic densities with respect to the Einstein gravity result. We perform some comparisons of the result with free fields calculations, strengthening previously observed universal properties of this function. We conclude in section 6 with some final comments and directions. Appendix A contains the proof of a couple of identities which we use in our derivation of the new functional in section 3 .

\subsection{Notation and conventions}

In the present paper we deal with various manifolds and metrics. Here we make some comments on our conventions and notation. We take indices in the $(d+1)$-dimensional bulk to be $\mu, \nu, \ldots$, and the bulk metric is denoted by $g_{\mu \nu}$. The entanglement entropy of a boundary region $A$ is computed as the integral of the entanglement functional on a spatial codimension-2 bulk surface homologous to $A$, which we call $\Gamma_{A}$. The induced metric on this surface is written as $h_{\mu \nu}$, and we will often have to deal with its extrinsic curvature, 
$K^{a}{ }_{\mu \nu}$. This is defined considering two orthonormal vectors to the surface $n_{a}{ }^{\mu}$, where indices $a, b, \ldots$ take values 1 and 2 :

$$
K_{\mu \nu}^{a} \equiv h_{\mu}^{\rho} h_{\nu}^{\sigma} \nabla_{\rho} n_{\sigma}^{a},
$$

and we assume an arbitrary extension of $n^{a}{ }_{\mu}$ to a neighborhood of the surface which keeps them normalized. Notice also that we work in Euclidean signature, which means $g_{\mu \nu} n_{a}{ }^{\mu} n_{b}{ }^{\nu}=\delta_{a b}$, and we define $n^{a}{ }_{\mu}=\delta^{a b} g_{\mu \nu} n_{b}{ }^{\nu}$. In particular, the induced metric can be written as

$$
h_{\mu \nu}=g_{\mu \nu}-n^{a}{ }_{\mu} n_{a \nu} .
$$

We also introduce projectors

$$
t_{i}^{\mu} \equiv \frac{\partial x^{\mu}}{\partial y^{i}},
$$

where indices $i, j, \ldots$ denote the tangent directions to the surface. Tensors with these kind of indices are always obtained by application of such projectors to their corresponding bulk tensors, e.g.,

$$
h_{i j} \equiv t_{i}^{\mu} t_{j}^{\nu} h_{\mu \nu}, \quad K_{i j}^{a} \equiv t_{i}^{\mu} t_{j}^{\nu} K_{\mu \nu}^{a} .
$$

We also define the binormal to the surface and the normal projector, respectively, as

$$
\epsilon_{\mu \nu} \equiv \epsilon_{a b} n^{a}{ }_{\mu} n^{b}{ }_{\nu}, \quad \perp_{\mu \nu} \equiv \delta_{a b} n^{a}{ }_{\mu} n^{b}{ }_{\nu},
$$

where $\epsilon_{a b}$ is the two-dimensional Levi-Civita symbol. In particular, this means that when indices $a, b, \ldots$ appear repeated in a tensorial structure the corresponding bulk tensor is contracted with the normal projector, namely

$$
V_{a}^{a} \equiv V^{\mu \nu} \perp_{\mu \nu}
$$

The binormal and the normal projector satisfy the useful relations

$$
\epsilon_{\mu \nu} \epsilon_{\rho \sigma}=2 \perp_{\mu[\rho} \perp_{\nu \mid \sigma]}, \quad g^{\mu \rho} \epsilon_{\mu \nu} \epsilon_{\rho \sigma}=\perp_{\nu \sigma}, \quad \epsilon_{\mu \nu} \epsilon^{\mu \nu}=2 .
$$

When performing generic computations of the entanglement functional we follow the conventions of $[32,46]$. This means that we take a particular set of adapted coordinates for $\Gamma_{A}$ so that

$$
\mathrm{d} s^{2}=\mathrm{d} z \mathrm{~d} \bar{z}+h_{i j} \mathrm{~d} y^{i} \mathrm{~d} y^{j},
$$

where $z \equiv \rho e^{i \tau}, \bar{z} \equiv \rho e^{-i \tau}$ are complex coordinates orthogonal to the surface. In these coordinates, the off-diagonal components $g_{z \bar{z}}=1 / 2$ and $g^{z \bar{z}}=2$ are the only non-vanishing part of the normal metric to the surface.

We take the cosmological constant to be negative throughout the paper, and write $-2 \Lambda \equiv d(d-1) / L^{2}$, so that the action scale $L$ coincides with the $\operatorname{AdS}_{d+1}$ radius, which we denote by $L_{\star}$, for Einstein gravity. For generic higher-curvature gravities, the equation which relates $L$ and $L_{\star}$ involves the corresponding higher-order couplings (it appears in eq. (5.2) below). Nevertheless, at leading order in the couplings - which is the setup we consider here - the two scales are equal to each other, $L=L_{\star}+\mathcal{O}\left(\alpha_{i}\right)$. We choose to present the results (mostly in section 5) in terms of the AdS radius $L_{\star}$. 


\section{GR splittings for perturbative higher-curvature theories}

Let us start considering the entanglement entropy for a region $A$ in some global state $\rho$ of some holographic CFT. This can be obtained as the $n \rightarrow 1$ limit of Rényi entropies $S_{n}(A)$, which in turn can be obtained via the replica trick as

$$
S_{n}(A)=-\frac{1}{n-1} \log \operatorname{Tr}\left(\rho_{A}^{n}\right)=-\frac{1}{n-1}\left(\log \mathcal{Z}_{n}-n \log \mathcal{Z}_{1}\right) .
$$

In this expression, $n$ is a positive integer, $\rho_{A}$ is the reduced density matrix of region $A$, and $\mathcal{Z}_{n}$ is the partition function of the field theory in the $n$-fold cover. In particular, $\mathcal{Z}_{1}$ is the partition function of the Euclidean manifold which, upon path integration, prepares the global state. In order to obtain the entanglement entropy $S_{\mathrm{EE}}(A)$ as the limit $n \rightarrow 1$ of the previous expression, an analytic continuation in $n$ is also needed.

Following the argument of [31], when the field theory has a gravity dual, in the saddlepoint approximation it is possible to identify $\log \mathcal{Z}_{n}=-I_{E}\left[B_{n}\right]$, where $I_{E}\left[B_{n}\right]$ is the Euclidean action of the gravitational theory evaluated at the bulk solution $B_{n}$ which is dual to the $n$-fold cover. This boundary geometry has a $\mathbb{Z}_{n}$ symmetry which interchanges the $n$ copies and, if this is respected in the bulk, we can consider the quotient $\hat{B}_{n}=B_{n} / \mathbb{Z}_{n}$, which is regular everywhere except at the codimension-2 bulk surface $\mathcal{C}_{n}$ consisting of the fixed points of $\mathbb{Z}_{n}$. Furthermore, the replica symmetry also guarantees that

$$
I_{E}\left[B_{n}\right]=n I_{E}\left[\hat{B}_{n}\right] .
$$

We can now analytically continue to non-integer $n$ this construction, and obtain the entanglement entropy as:

$$
S_{\mathrm{HEE}}(A)=\lim _{n \rightarrow 1} \frac{n}{n-1}\left(I_{E}\left[\hat{B}_{n}\right]-I_{E}\left[B_{1}\right]\right)=\left.\partial_{n} I_{E}\left[\hat{B}_{n}\right]\right|_{n=1} .
$$

Since $I_{E}\left[B_{1}\right]$ is a bulk solution to the equations of motion, this variation away from $n=1$ might seem to vanish. This is not the case because when we vary $n$ we change the opening angle of the conical defect at $\mathcal{C}_{n}$, and this region has to be excluded from the action integral, introducing a boundary where conditions change with $n$. Details of this procedure can be found e.g., in [32]. The relevant fact is that the computation of the entanglement entropy gets reduced to the evaluation of the on-shell Euclidean action of the gravitational theory in the conical defect $\mathcal{C}_{n}$. The opening angle of this defect is $2 \pi / n$, and after obtaining the contributions to the action we must take an $n$-derivative at $n=1$.

In order to compute $S_{\mathrm{HEE}}(A)$ we need to evaluate the action of a given gravitational theory for a bulk geometry which regulates the conical singularity. This is a rather technical task, but there is a key point which was initially overlooked in [32, 33]: there are many ways in which a conical defect can be regulated [42, 44-46]. Different prescriptions produce different functionals. This ambiguity is usually called the "splitting problem". The particular gravitational theory of interest should determine the correct one through its equations of motion [43, 46].

When interested in perturbative higher-curvature corrections to Einstein gravity, the appropriate splittings were obtained in [46]. At first order in the higher-order couplings one 
can simply regulate using Einstein's equations. This is so because the particular regularization does not affect the Einstein gravity term in (2.3) (it always produces the usual area law), and the higher curvature terms in the action are already first order in the couplings. As a consequence, corrections to the regulated geometry coming from modifications to the equations of motion are second order in the action.

All in all, the expression for the holographic entanglement entropy for a perturbative higher-curvature gravity with Euclidean action $\mathcal{L}_{E}\left(g_{\mu \nu}, R_{\mu \nu \rho \sigma}\right)$ is given by

$$
S_{\mathrm{HEE}}(A)=2 \pi \int_{\Gamma_{A}} \mathrm{~d}^{D-2} y \sqrt{h}\left[\frac{\partial \mathcal{L}_{E}}{\partial R_{z \bar{z} z \bar{z}}}+\sum_{\alpha}\left(\frac{\partial^{2} \mathcal{L}_{E}}{\partial R_{z i z j} \partial R_{\bar{z} k \bar{z} l}}\right)_{\alpha} \frac{8 K_{z i j} K_{\bar{z} k l}}{q_{\alpha}+1}\right],
$$

where $\Gamma_{A}$ is just the RT surface and the prescription for the $\alpha$-sum is unambiguously determined - see below. The area term in the previous equation - coming from the Einstein gravity part of the action - is stationary for the RT surface, and therefore first order variations of the surface will not change its value. On the other hand, contributions of higher-order terms to the previous functional will already be first-order in the couplings, and thus insensitive to first-order modifications of the surface.

As we mentioned before, there are in principle different ways to regulate the conical singularity, which give rise to different prescriptions for the $\alpha$ sum. On general grounds, the idea is the following. The second derivative of the Lagrangian will be a sum of terms which are monomials with different contractions of components of the Riemann tensor. These contractions are to be expanded in terms of their $z$ and $\bar{z}$ indices, obtaining an expression of the second derivative of the Lagrangian involving only $R_{z \bar{z} z \bar{z}}, R_{z \bar{z} z i}, R_{z \bar{z} i j}, R_{z i \bar{z} j}, R_{z i z j}$, $R_{z i j k}, R_{i j k l}$, plus components related to these by complex conjugation of the indices. ${ }^{7}$ After this is done, each regularization of the conical defect will provide a "splitting": a rule to divide each of the previous components of the Riemann tensor schematically as

$$
R_{M I}=\tilde{R}_{M I}+\mathcal{K}_{M I}
$$

In this expression, $M$ labels the different components of the Riemann tensor enumerated before, while $I$ is a generalized index containing all the $i, j, k, \ldots$ indices of the particular component under consideration (which might be none). This expansion has to be performed in all the components of the Riemann tensor, and once this is done, each of the resulting monomials is labelled by $\alpha$. The splitting provides also a value $q_{\alpha}$ for each $\mathcal{K}_{M I}$. In each term we have a definite value of $q_{\alpha}$, given by the sum of the values of all the $\mathcal{K}_{M I}$ in that monomial. Expression (2.4) instructs us then to divide each term by $q_{\alpha}+1$. Once this is done, we can eliminate the $\tilde{R}_{M I}$ (which are auxiliary objects in this construction whose particular geometrical meaning is irrelevant as far as the functional construction is concerned) in favor of the Riemann tensor components by using (2.5) again.

\footnotetext{
${ }^{7}$ Notice that components of the Ricci tensor and the Ricci scalar have to be expanded in terms of these basic objects as well. For instance, we would write

$$
R_{z \bar{z}}=g^{\mu \nu} R_{z \mu \bar{z} \nu}=-2 R_{z \bar{z} z \bar{z}}+g^{i j} R_{z i \bar{z} j} .
$$
}


The particular example of (2.5) relevant for our purposes comes from the regularization of the conical defect imposed by Einstein's equations, which is valid for any theory containing perturbative corrections to Einstein gravity in the action. In such a case, the splittings take the form

$$
\begin{aligned}
R_{z \bar{z} z \bar{z}} & =\tilde{R}_{z \bar{z} z \bar{z}}-\frac{1}{8} K^{a i j} K_{a i j}, \\
R_{z \bar{z} i j} & =\tilde{R}_{z \bar{z} i j}-2 K_{z[i \mid}{ }^{k} K_{\bar{z} \mid j] k} \\
R_{z i \bar{z} j} & =\tilde{R}_{z i \bar{z} j}-K_{z i}{ }^{k} K_{\bar{z} j k} \\
R_{i j k l} & =\tilde{R}_{i j k l}-2 K_{a i[k} K^{a}{ }_{l] j}
\end{aligned}
$$

with the remaining components having a trivial splitting, i.e., $\tilde{R}_{M I}=0$ for them. The values of $q_{\alpha}$ are: $q_{\alpha}=1$ for any of the previous terms quadratic in extrinsic curvatures, $q_{\alpha}=1$ for $R_{z i z j}$ (and its complex conjugate), and $q_{\alpha}=1 / 2$ for $R_{z i j k}$ and $R_{z \bar{z} z i}$ (and their complex conjugates).

All in all, this complicated procedure is nothing but a way to generate contributions to the holographic entanglement entropy functional containing higher and higher powers of the extrinsic curvature. One of the main results in this paper will consist in reinterpreting and rewriting this algorithm in a more transparent way, making manifest this generation of terms with an increasing number of powers of $K$.

\section{Rewriting the HEE functional}

In this section we perform a rewriting of the holographic entanglement entropy functional for higher-curvature gravities. We manage to write it completely in terms of explicit contractions of extrinsic curvatures and derivatives with respect to Riemann tensors, getting rid of the weighted sum over $\alpha$ appearing in the anomaly piece. We do this for the Riemann tensor splittings corresponding to Einstein gravity, which allows us to produce a new general expression valid for arbitrary higher-curvature theories at leading order in the corresponding couplings. The structure of the expression is particularly simple for densities up to quartic order in curvature, and we provide new explicit formulas for cubic and quartic theories. Applied to the case of Lovelock theories, our formula for the corresponding anomaly piece can be suggestively written in terms of an exponential of the derivative of the only component of the Riemann tensor which is relevant in that case, contracted with two extrinsic curvatures. We also perform a hopefully illustrative application of our formulas to a particular monomial coming from putative quintic densities showing how it agrees with the result obtained via the $\alpha$ sum.

\subsection{Symmetry factors in derivatives and some notation}

Let us start by making a couple of comments regarding how to take derivatives with respect to Riemann tensor components and introducing some notation which we will be using throughout this section. 
The issues discussed here arise due to the conventional definition of the derivative with respect to the Riemann tensor:

$$
\frac{\partial R_{\mu \nu \rho \sigma}}{\partial R_{\alpha \beta \gamma \delta}} \equiv \frac{1}{2}\left[\delta_{[\mu}^{\alpha} \delta_{\nu]}^{\beta} \delta_{[\rho}^{\gamma} \delta_{\sigma]}^{\delta}+\delta_{[\rho}^{\alpha} \delta_{\sigma]}^{\beta} \delta_{[\mu}^{\gamma} \delta_{\nu]}^{\delta}\right]
$$

This definition respects the symmetries of the Riemann tensor and, at the same time, it has the following nice (and expected) property,

$$
R_{\alpha \beta \gamma \delta} \frac{\partial R_{\mu \nu \rho \sigma}}{\partial R_{\alpha \beta \gamma \delta}}=R_{\mu \nu \rho \sigma},
$$

which will be key when performing Taylor-like expansions of functions of the Riemann tensor.

Some care must be taken, however, when singling out specific components of the Riemann tensor. For instance, using the previous definition one finds

$$
\frac{\partial R_{z \bar{z} i j}}{\partial R_{z \bar{z} k l}}=\frac{1}{2}\left[\delta_{[z}^{z} \delta_{\bar{z}]}^{\bar{z}} \delta_{[i}^{k} \delta_{j]}^{l}+\delta_{[i}^{z} \delta_{j]}^{\bar{z}} \delta_{[z}^{k} \delta_{\bar{z}]}^{l}\right]=\frac{1}{4} \delta_{[i}^{k} \delta_{j]}^{l},
$$

which leads to

$$
R_{z \bar{z} k l} \frac{\partial R_{z \bar{z} i j}}{\partial R_{z \bar{z} k l}}=\frac{1}{4} R_{z \bar{z} i j} .
$$

The factor $1 / 4$ arises from the different positions in which we can put the $z, \bar{z}$ indices using the symmetries of the Riemann tensor, $R_{z \bar{z} k l}, R_{\bar{z} z k l}, R_{k l z \bar{z}}$, and $R_{k l \bar{z} z}$. Something analogous happens for the rest of components of the Riemann tensor. Hence, whenever performing Taylor-like expansions in terms of such components we will need to take these extra factors into account. In order to do so, it will prove useful to define a new derivative operator, $\hat{\partial}$, which already includes them. The definitions for the different components read

$$
\begin{aligned}
\frac{\hat{\partial}}{\hat{\partial} R_{z \bar{z} z \bar{z}}} \equiv 4 \frac{\partial}{\partial R_{z \bar{z} z \bar{z}}}, & \frac{\hat{\partial}}{\hat{\partial} R_{z \bar{z} z i}} \equiv 8 \frac{\partial}{\partial R_{z \bar{z} z i}}, & \frac{\hat{\partial}}{\hat{\partial} R_{z \bar{z} i j}} \equiv 4 \frac{\partial}{\partial R_{z \bar{z} i j}}, \\
\frac{\hat{\partial}}{\hat{\partial} R_{z i z j}} \equiv 4 \frac{\partial}{\partial R_{z i z j}}, & \frac{\hat{\partial}}{\hat{\partial} R_{z i \bar{z} j}} \equiv 8 \frac{\partial}{\partial R_{z i \bar{z} j}}, & \frac{\partial}{\hat{\partial} R_{z i j k}} \equiv 4 \frac{\partial}{\partial R_{z i j k}}, \\
\frac{\hat{\partial}}{\hat{\partial} R_{i j k l}} \equiv \frac{\partial}{\partial R_{i j k l}} . & &
\end{aligned}
$$

The remaining ones can be obtained by complex conjugation.

Below we will manipulate expressions involving multiple derivatives with respect to all these components of the Riemann tensor. In order to do that, it is convenient to introduce some notation which allows us to represent them in a compact form. Firstly, let us define upper case latin indices $I, J, \ldots$ to collect all $i, j, k, \ldots$ indices that might appear in a given tensor. Similarly, we introduce $M, N, \ldots$ indices to represent the different Riemann tensor components involving $z$ and $\bar{z}$ indices. In practice, we just want this notation to perform 
Taylor expansions, for which the relevant thing to keep in mind is the following compact definition

$$
\begin{aligned}
R_{M I} \hat{\partial}^{M I} \equiv & +R_{z \bar{z} z \bar{z}} \frac{\hat{\partial}}{\hat{\partial} R_{z \bar{z} z \bar{z}}}+R_{z \bar{z} i j} \frac{\hat{\partial}}{\hat{\partial} R_{z \bar{z} i j}}+R_{z i \bar{z} j} \frac{\hat{\partial}}{\hat{\partial} R_{z i \bar{z} j}}+R_{i j k l} \frac{\hat{\partial}}{\hat{\partial} R_{i j k l}} \\
& +\left[R_{z \bar{z} z i} \frac{\hat{\partial}}{\hat{\partial} R_{z \bar{z} z i}}+R_{z i z j} \frac{\hat{\partial}}{\hat{\partial} R_{z i z j}}+R_{z i j k} \frac{\hat{\partial}}{\hat{\partial} R_{z i j k}}+\text { c.c. }\right]
\end{aligned}
$$

where c.c. stands for the complex conjugate components of the terms in the parentheses (which are the only ones that have a different number of $z$ and $\bar{z}$ indices). This can be thought of as a sum over $M$ (the $z$ and $\bar{z}$ indices) and then, for each $M$, an extra sum over tangent indices $I$. Note that for $R_{z \bar{z} z \bar{z}}$ the second sum does not exist, and in that case $I$ represents an empty set of tangent indices.

As we explained in the previous section, different components of the Riemann tensor have different splitting structures. In general, any component splits as in eq. (2.5) where $\tilde{R}_{M I}$ has $q_{\alpha}=0$ and $\mathcal{K}_{M I}$ has $q_{\alpha} \neq 0$. The $q_{\alpha}$ for the $\mathcal{K}_{M I}$ piece can take two values. Components $R_{z \bar{z} z \bar{z}}, R_{z \bar{z} i j}, R_{z i \bar{z} j}, R_{z i z j}, R_{\bar{z} i \bar{z} j}$, and $R_{i j k l}$ have $q_{\alpha}=1$ for that part, and we will generically refer to them with labels $A, A^{\prime}, \ldots$ On the other hand, components $R_{z i j k}, R_{\bar{z} i j k}, R_{z \bar{z} z i}$, and $R_{\bar{z} z \bar{z} i}$ have $q_{\alpha}=1 / 2$ for the $\mathcal{K}_{M I}$ part and we will refer to them with labels $B, B^{\prime}, \ldots$ In terms of these, the operator (3.8) splits into two contributions:

$$
\begin{aligned}
R_{A I} \hat{\partial}^{A I} \equiv & +R_{z \bar{z} z \bar{z}} \frac{\hat{\partial}}{\hat{\partial} R_{z \bar{z} z \bar{z}}}+R_{z \bar{z} i j} \frac{\hat{\partial}}{\hat{\partial} R_{z \bar{z} i j}}+R_{z i \bar{z} j} \frac{\hat{\partial}}{\hat{\partial} R_{z i \bar{z} j}}+R_{i j k l} \frac{\hat{\partial}}{\hat{\partial} R_{i j k l}} \\
& +\left[R_{z i z j} \frac{\hat{\partial}}{\hat{\partial} R_{z i z j}}+\text { c.c. }\right] \\
R_{B I} \hat{\partial}^{B I} \equiv & {\left[R_{z i j k} \frac{\hat{\partial}}{\hat{\partial} R_{z i j k}}+R_{z \bar{z} z i} \frac{\hat{\partial}}{\hat{\partial} R_{z \bar{z} z i}}+\text { c.c. }\right] . }
\end{aligned}
$$

\subsection{New form of the HEE functional}

Equipped with this notation, we are ready to start rewriting the anomaly piece in the holographic entanglement entropy functional. The $\alpha$ expansion appearing in that term is performed on the following object, for which we define the shorthand notation

$$
\left(\frac{8 \partial^{2} \mathcal{L}_{E}}{\partial \operatorname{Riem}^{2}} K^{2}\right) \equiv 8 \frac{\partial^{2} \mathcal{L}_{E}}{\partial R_{z i z j} \partial R_{\bar{z} k \bar{z} l}} K_{z i j} K_{\bar{z} k l} .
$$

This object is a complicated expression involving the different Riemann tensor components. Once we have it for a given theory, we have to apply an splitting of the form (2.5) to each component, account for the $q_{\alpha}$ value of each monomial and divide it by $\left(1+q_{\alpha}\right)$.

In order to understand the steps we will follow, it is illustrative to consider first a simplified version of the problem. Suppose we have some function $f(x)$ and we want to substitute $x=\tilde{x}+k$ in a way such that we explicitly isolate monomials depending on the 
number of $k$ factors they have. A simple way to do this is to Taylor-expand $f(\tilde{x}+k)$ around $x=0$, namely,

$$
f(\tilde{x}+k)=\sum_{n=0}^{\infty} \frac{1}{n !}(\tilde{x}+k)^{n} f^{(n)}(0),
$$

and then apply the binomial theorem to $(\tilde{x}+k)^{n}$ to isolate terms with a definite number of $k$ factors. Notice also that, if we wish to avoid evaluating derivatives at 0 , we can also Taylor-expand the derivative around a general point $x$,

$$
f^{(n)}(0)=\sum_{m=0}^{\infty} \frac{1}{m !}(0-x)^{m} f^{(n+m)}(x)=\sum_{m=0}^{\infty} \frac{1}{m !}(-x)^{m} f^{(n+m)}(x) .
$$

Observe that, despite its appearance, this expression does not really depend on $x$. Putting the pieces together, we see that counting the number of $k$ 's in each monomial appearing in $f(\tilde{x}+k)$ amounts to expanding the binomial $(\tilde{x}+k)^{n}$ in the expression

$$
f(\tilde{x}+k)=\sum_{n, m=0}^{\infty} \frac{1}{n ! m !}(\tilde{x}+k)^{n}(-x)^{m} f^{(n+m)}(x),
$$

where we emphasize that the $x$ 's in the right-hand side are not to be substituted by $\tilde{x}+k$. In the above expression we can pair each of the $n+m$ derivatives with the factors $(\tilde{x}+k)$ and $x$ provided we introduce some ordering convention. The idea is to impose that derivatives only act on $f(x)$, and not on explicit $x$ factors,

$$
f(\tilde{x}+k)=\left[: \sum_{n, m=0}^{\infty} \frac{1}{n ! m !}\left[(\tilde{x}+k) \partial_{x}\right]^{n}\left(-x \partial_{x}\right)^{m}:\right] f(x) .
$$

This notation will turn out to be convenient when dealing with the analogous expressions involving Riemann tensor components.

Now, with some care, the idea presented above can be extended to functions of several variables. In the case of interest here, these variables will be Riemann tensor components. Roughly speaking, $f(x)$ will be replaced by the object defined in (3.11) and $x=\tilde{x}+k$ will be the splitting of each component, $R_{M I}=\tilde{R}_{M I}+\mathcal{K}_{M I}$. The first step is the expansion around 0 , which gives an expression in which such splitting is already applied

$$
\begin{aligned}
\left(\frac{8 \partial^{2} \mathcal{L}_{E}}{\partial \operatorname{Riem}^{2}} K^{2}\right)=\sum_{n=0}^{\infty} \frac{1}{n !} & \left(\tilde{R}_{M_{1} I_{1}}+\mathcal{K}_{M_{1} I_{1}}\right) \ldots\left(\tilde{R}_{M_{n} I_{n}}+\mathcal{K}_{M_{n} I_{n}}\right) \\
& \times\left[\frac{\hat{\partial}}{\hat{\partial} R_{M_{1} I_{1}} \ldots \hat{\partial} R_{M_{n} I_{n}}}\left(\frac{8 \partial^{2} \mathcal{L}_{E}}{\partial \operatorname{Riem}^{2}} K^{2}\right)\right]_{\text {Riem }=0}
\end{aligned}
$$

Just like for $f^{(n)}(0)$ above, the derivatives piece at zero can be traded by derivatives at a general value of the Riemann tensor as

$$
\begin{aligned}
& {\left[\frac{\hat{\partial}}{\hat{\partial} R_{M_{1} I_{1}} \ldots \hat{\partial} R_{M_{n} I_{n}}}\left(\frac{8 \partial^{2} \mathcal{L}_{E}}{\partial \operatorname{Riem}^{2}} K^{2}\right)\right]_{\text {Riem }=0}=} \\
& \sum_{m=0}^{\infty} \frac{1}{m !}\left(-R_{N_{1} J_{1}}\right) \ldots\left(-R_{N_{m} J_{m}}\right) \frac{\hat{\partial}}{\hat{\partial} R_{N_{1} J_{1}} \ldots \hat{\partial} R_{N_{m} J_{m}} \hat{\partial} R_{M_{1} I_{1}} \ldots \hat{\partial} R_{M_{n} I_{n}}}\left(\frac{8 \partial^{2} \mathcal{L}_{E}}{\partial \operatorname{Riem}^{2}} K^{2}\right) .
\end{aligned}
$$


The two previous expressions can be combined into a single and simpler one if we introduce again a sort of normal ordering prescription for derivatives. By this we mean:

$$
:\left(R_{M I} \hat{\partial}^{M I}\right)^{n}: \equiv R_{M_{1} I_{1}} \ldots R_{M_{n} I_{n}} \frac{\hat{\partial}}{\hat{\partial} R_{M_{1} I_{1}} \ldots \hat{\partial} R_{M_{n} I_{n}}},
$$

so that derivatives only act on the object completely to the right of the expression. Then we have

$$
\left(\frac{8 \partial^{2} \mathcal{L}_{E}}{\partial \operatorname{Riem}^{2}} K^{2}\right)=\left[: \sum_{n, m=0}^{\infty} \frac{1}{n ! m !}\left(\left(\tilde{R}_{M I}+\mathcal{K}_{M I}\right) \hat{\partial}^{M I}\right)^{n}\left(-R_{N J} \hat{\partial}^{N J}\right)^{m}:\right]\left(\frac{8 \partial^{2} \mathcal{L}_{E}}{\partial \operatorname{Riem}^{2}} K^{2}\right) .
$$

From now on, we will work with the operator between brackets alone, since it contains all we need, namely, the explicit dependence on the $\mathcal{K}_{M I}$. We will also implicitly assume the normal ordering convention for derivatives.

Now, let us use the following useful identity

$$
\sum_{n, m=0}^{\infty} f(n, m)=\sum_{S=0}^{\infty} \sum_{n=0}^{S} f(n, S-n)
$$

to collect terms in the sums depending on the total number of derivatives they have, $S=n+m$. This gives:

$$
\begin{aligned}
& \sum_{S=0}^{\infty} \sum_{n=0}^{S} \frac{1}{n !(S-n) !}\left(\left(\tilde{R}_{M I}+\mathcal{K}_{M I}\right) \hat{\partial}^{M I}\right)^{n}\left(-R_{N J} \hat{\partial}^{N J}\right)^{S-n} \\
& =\sum_{S=0}^{\infty} \frac{1}{S !}\left(\left(\tilde{R}_{M I}+\mathcal{K}_{M I}-R_{M I}\right) \hat{\partial}^{M I}\right)^{S}
\end{aligned}
$$

where we have applied the binomial theorem. ${ }^{8}$ Let us pause for a moment and look at eq. (3.21). Here we could be tempted to use $\tilde{R}_{M I}+\mathcal{K}_{M I}-R_{M I}=0$, which would mean that the previous operator is simply the identity (because of the $S=0$ term). This is not a contradiction. As a matter of fact, the only thing we have done so far is applying the identity in an elaborated way. But we have achieved our goal, since we have isolated the appearances of $\mathcal{K}_{M I}$ in the $\alpha$-expansion: all these factors are the ones explicitly appearing in the previous expression.

From now on, we will have to deal separately with the two types of Riemann tensor components: those we called type $A$ (with $q_{\alpha}=1$ for the corresponding $\mathcal{K}_{M I}$ ) and those we called type $B$ (with $q_{\alpha}=1 / 2$ for the corresponding $\mathcal{K}_{M I}$ ). This can be easily done from the previous expression,

$$
\begin{aligned}
& \sum_{S=0}^{\infty} \frac{1}{S !}\left(\left(\tilde{R}_{A I}+\mathcal{K}_{A I}-R_{A I}\right) \hat{\partial}^{A I}+\left(\tilde{R}_{B I}+\mathcal{K}_{B I}-R_{B I}\right) \hat{\partial}^{B I}\right)^{S}= \\
& \sum_{S=0}^{\infty} \sum_{T=0}^{S} \frac{1}{T !(S-T) !}\left[\left(\tilde{R}_{A I}+\mathcal{K}_{A I}-R_{A I}\right) \hat{\partial}^{A I}\right]^{T}\left[\left(\tilde{R}_{B J}+\mathcal{K}_{B J}-R_{B J}\right) \hat{\partial}^{B J}\right]^{S-T}
\end{aligned}
$$

${ }^{8}$ For this to be valid, we need the elements inside the parentheses to commute with each other. This is guaranteed by the normal ordering prescription for derivatives. 
The next step is to isolate the number of $\mathcal{K}$ 's of each type, to prepare for the $\left(1+q_{\alpha}\right)$ division,

$$
\begin{aligned}
& \sum_{S=0}^{\infty} \sum_{T=0}^{S} \sum_{\lambda_{1}=0}^{T} \sum_{\lambda_{2}=0}^{S-T} \frac{1}{T !(S-T) !} \frac{T !}{\lambda_{1} !\left(T-\lambda_{1}\right) !}\left[\mathcal{K}_{A I} \hat{\partial}^{A I}\right]^{\lambda_{1}}\left[\left(\tilde{R}_{A^{\prime} I^{\prime}}-R_{A^{\prime} I^{\prime}}\right) \hat{\partial}^{A^{\prime} I^{\prime}}\right]^{T-\lambda_{1}} \\
& \frac{(S-T) !}{\lambda_{2} !\left(S-T-\lambda_{2}\right) !}\left[\mathcal{K}_{B J} \hat{\partial}^{B J}\right]^{\lambda_{2}}\left[\left(\tilde{R}_{B^{\prime} J^{\prime}}-R_{B^{\prime} J^{\prime}}\right) \hat{\partial}^{B^{\prime} J^{\prime}}\right]^{S-T-\lambda_{2}}
\end{aligned}
$$

In this expression, it is manifest that we have $\lambda_{1}$ components $\mathcal{K}_{A I}$, which contribute 1 to $q_{\alpha}$, and $\lambda_{2}$ components $\mathcal{K}_{B J}$, which contribute $1 / 2$ to $q_{\alpha}$. Hence, we are ready to divide by $\left(1+q_{\alpha}\right)=\left(1+\lambda_{1}+\lambda_{2} / 2\right)$, obtaining

$$
\begin{gathered}
\sum_{S=0}^{\infty} \sum_{T=0}^{S} \sum_{\lambda_{1}=0}^{T} \sum_{\lambda_{2}=0}^{S-T} \frac{2}{\left(2+2 \lambda_{1}+\lambda_{2}\right)} \frac{1}{\lambda_{1} !\left(T-\lambda_{1}\right) !}\left[\mathcal{K}_{A I} \hat{\partial}^{A I}\right]^{\lambda_{1}}\left[\left(\tilde{R}_{A^{\prime} I^{\prime}}-R_{A^{\prime} I^{\prime}}\right) \hat{\partial}^{A^{\prime} I^{\prime}}\right]^{T-\lambda_{1}} \\
\frac{1}{\lambda_{2} !\left(S-T-\lambda_{2}\right) !}\left[\mathcal{K}_{B J} \hat{\partial}^{B J}\right]^{\lambda_{2}}\left[\left(\tilde{R}_{B^{\prime} J^{\prime}}-R_{B^{\prime} J^{\prime}}\right) \hat{\partial}^{B^{\prime} J^{\prime}}\right]^{S-T-\lambda_{2}}
\end{gathered}
$$

At this point, the $\alpha$-sum has been performed, and we do not need to explicitly keep the $\mathcal{K}$ dependence isolated. We can also rewrite the $\tilde{R}$ back in terms of conventional Riemann tensor components. Using $\tilde{R}_{M I}=R_{M I}-\mathcal{K}_{M I}$ we have

$$
\sum_{S=0}^{\infty} \sum_{T=0}^{S} \sum_{\lambda_{1}=0}^{T} \sum_{\lambda_{2}=0}^{S-T} \frac{2}{\left(2+2 \lambda_{1}+\lambda_{2}\right)} \frac{(-1)^{S-\lambda_{1}-\lambda_{2}}}{\lambda_{1} !\left(T-\lambda_{1}\right) !} \frac{1}{\lambda_{2} !\left(S-T-\lambda_{2}\right) !}\left(\mathcal{K}_{A I} \hat{\partial}^{A I}\right)^{T}\left(\mathcal{K}_{B J} \hat{\partial}^{B J}\right)^{S-T}
$$

At this point we proceed to perform the $\lambda_{1}$ and $\lambda_{2}$ sums, which do not affect the derivative operators. Let us start with the $\lambda_{2}$ one. It is possible to show that

$$
\sum_{\lambda=0}^{S-T} \frac{(-1)^{\lambda}}{\left(2+2 \lambda_{1}+\lambda\right)} \frac{1}{\lambda !(S-T-\lambda) !}=\frac{\left(2 \lambda_{1}+1\right) !}{\left(2 \lambda_{1}+2+S-T\right) !} .
$$

Detailed derivations of this identity as well as of eq. (3.28) are included in appendix A. After performing this sum, the operator becomes:

$$
2 \sum_{S=0}^{\infty} \sum_{T=0}^{S} \sum_{\lambda_{1}=0}^{T} \frac{(-1)^{S-\lambda_{1}}}{\lambda_{1} !\left(T-\lambda_{1}\right) !} \frac{\left(2 \lambda_{1}+1\right) !}{\left(2 \lambda_{1}+2+S-T\right) !}\left(\mathcal{K}_{A I} \hat{\partial}^{A I}\right)^{T}\left(\mathcal{K}_{B J} \hat{\partial}^{B J}\right)^{S-T} .
$$

We can now try to do the $\lambda_{1}$ one. We find the following integral representation of the sum, ${ }^{9}$

$$
\sum_{\lambda=0}^{T} \frac{(-1)^{\lambda}}{\lambda !(T-\lambda) !} \frac{(2 \lambda+1) !}{(2 \lambda+2+S-T) !}=\frac{1}{T !(S-T) !} \int_{0}^{1} \mathrm{~d} u u\left(1-u^{2}\right)^{T}(1-u)^{S-T} .
$$

\footnotetext{
${ }^{9}$ This can be explicitly written in terms of Gauss' hypergeometric function as

$$
\sum_{\lambda=0}^{T} \frac{(-1)^{\lambda}}{\lambda !(T-\lambda) !} \frac{(2 \lambda+1) !}{(2 \lambda+2+S-T) !}=\frac{2+S-(S-T){ }_{2} F_{1}[1,-T ; 3+S ;-1]}{2(1+S)(2+S)(S-T) ! T !}
$$
}

but the integral form turns out to be more useful for our purposes. 
Continuing from (3.27), we have

$$
\begin{gathered}
\int_{0}^{1} \mathrm{~d} u 2 u \sum_{S=0}^{\infty} \sum_{T=0}^{S} \frac{(-1)^{S}}{T !(S-T) !}\left(\left(1-u^{2}\right) \mathcal{K}_{A I} \hat{\partial}^{A I}\right)^{T}\left((1-u) \mathcal{K}_{B J} \hat{\partial}^{B J}\right)^{S-T} \\
=\sum_{S=0}^{\infty} \frac{(-1)^{S}}{S !} \int_{0}^{1} \mathrm{~d} u 2 u\left[\left(1-u^{2}\right) \mathcal{K}_{A I} \hat{\partial}^{A I}+(1-u) \mathcal{K}_{B J} \hat{\partial}^{B J}\right]^{S}
\end{gathered}
$$

This is our final result. Let us collect everything here, including the definitions needed to interpret it. We have found that the anomaly term in the holographic entanglement entropy functional can be written as

$$
\begin{aligned}
& \sum_{\alpha} \frac{1}{1+q_{\alpha}}\left(\frac{8 \partial^{2} \mathcal{L}_{E}}{\partial \operatorname{Riem}^{2}} K^{2}\right)_{\alpha} \\
& \quad=\sum_{S=0}^{\infty} \frac{1}{S !} \int_{0}^{1} \mathrm{~d} u 2 u:\left[-\left(1-u^{2}\right) \mathcal{K}_{A I} \hat{\partial}^{A I}-(1-u) \mathcal{K}_{B J} \hat{\partial}^{B J}\right]^{S}:\left(\frac{8 \partial^{2} \mathcal{L}_{E}}{\partial \operatorname{Riem}^{2}} K^{2}\right)
\end{aligned}
$$

where we emphasized again that derivatives have to be taken after normal ordering and

$$
\begin{aligned}
\mathcal{K}_{A I} \hat{\partial}^{A I} \equiv & -\frac{1}{2} K^{a i j} K_{a i j} \frac{\partial}{\partial R_{z \bar{z} z \bar{z}}}-8 K_{z i}{ }^{k} K_{\bar{z} j k} \frac{\partial}{\partial R_{z \bar{z} i j}}-8 K_{z i}{ }^{k} K_{\bar{z} j k} \frac{\partial}{\partial R_{z i \bar{z} j}} \\
& -2 K_{a i k} K^{a}{ }_{l j} \frac{\partial}{\partial R_{i j k l}}+\left(4 R_{z i z j} \frac{\partial}{\partial R_{z i z j}}+\text { c.c. }\right), \\
\mathcal{K}_{B J} \hat{\partial}^{B J} \equiv & \left(4 R_{z i j k} \frac{\partial}{\partial R_{z i j k}}+8 R_{z \bar{z} z i} \frac{\partial}{\partial R_{z \bar{z} z i}}+\text { c.c. }\right) .
\end{aligned}
$$

Observe that the sum in eq. (3.30) can be formally performed, allowing us to write the result in an exponential form

$$
\sum_{\alpha} \frac{1}{1+q_{\alpha}}\left(\frac{8 \partial^{2} \mathcal{L}_{E}}{\partial \operatorname{Riem}^{2}} K^{2}\right)_{\alpha}=\int_{0}^{1} \mathrm{~d} u 2 u \mathrm{e}^{-F(u)}\left(\frac{8 \partial^{2} \mathcal{L}_{E}}{\partial \operatorname{Riem}^{2}} K^{2}\right),
$$

where

$$
F(u) \equiv\left[\left(1-u^{2}\right) \mathcal{K}_{A I} \hat{\partial}^{A I}+(1-u) \mathcal{K}_{B J} \hat{\partial}^{B J}\right] .
$$

In subsection 3.7 below we present a covariant version of these new formulas. Observe that even though the anomaly term naively involves the contraction of intrinsic curvatures with two extrinsic curvatures, it is manifest from our formula that the sum over $\alpha$ hides possible contractions with an arbitrary (even) number of extrinsic curvatures - in particular, order $n$ densities will produce terms involving up to $2(n-1)$ extrinsic curvatures.

There are some obvious particular cases in which the above expression simplifies considerably. Firstly, if no $B$ type terms appear in the second derivative of the Lagrangian, we can write

$$
\begin{aligned}
\sum_{\alpha} \frac{1}{1+q_{\alpha}}\left(\frac{8 \partial^{2} \mathcal{L}_{E}}{\partial \operatorname{Riem}^{2}} K^{2}\right)_{\alpha} & =\sum_{S=0}^{\infty} \frac{1}{(S+1) !}\left(-\mathcal{K}_{A I} \hat{\partial}^{A I}\right)^{S}\left(\frac{8 \partial^{2} \mathcal{L}_{E}}{\partial \operatorname{Riem}^{2}} K^{2}\right) \\
& =\left[\mathcal{K}_{A I} \hat{\partial}^{A I}\right]^{-1}\left[1-\mathrm{e}^{-\mathcal{K}_{A I} \hat{\partial}^{A I}}\right]\left(\frac{8 \partial^{2} \mathcal{L}_{E}}{\partial \operatorname{Riem}^{2}} K^{2}\right)
\end{aligned}
$$


Similarly, if only type $B$ terms were present, the result would simplify to

$$
\begin{aligned}
\sum_{\alpha} \frac{1}{1+q_{\alpha}}\left(\frac{8 \partial^{2} \mathcal{L}_{E}}{\partial \operatorname{Riem}^{2}} K^{2}\right)_{\alpha} & =\sum_{S=0}^{\infty} \frac{2}{(S+2) !}\left(-\mathcal{K}_{B I} \hat{\partial}^{B I}\right)^{S}\left(\frac{8 \partial^{2} \mathcal{L}_{E}}{\partial \operatorname{Riem}^{2}} K^{2}\right) \\
& =-2\left[\mathcal{K}_{B I} \hat{\partial}^{B I}\right]^{-2}\left[1-\mathcal{K}_{B I} \hat{\partial}^{B I}-\mathrm{e}^{-\mathcal{K}_{B I} \hat{\partial}^{B I}}\right]\left(\frac{8 \partial^{2} \mathcal{L}_{E}}{\partial \operatorname{Riem}^{2}} K^{2}\right) .
\end{aligned}
$$

As we will see in a moment, there is at least one important case for which only type $A$ terms appear, namely, Lovelock theories. It is harder to imagine how only type $B$ terms could appear. Nevertheless, the result obtained here will prove to be useful for presenting the explicit form of the anomaly term for cubic and quartic theories.

Before closing this subsection, let us mention that, while our new formulas have been obtained assuming a particular splitting for the Riemann tensor components - namely, the one valid for perturbative higher-curvature gravities summarized in eq. (2.5) - an analogous procedure to the one presented here should allow to produce similar expressions for other possible splittings.

\subsection{Anomaly term in Lovelock theories}

Lovelock gravities $[47,48]$ are special in many respects - see also subsection 4.2 below. In particular, as argued in [32], the object (3.11) only contains a single kind of Riemann tensor component for them, namely, $R_{i j k l}$. The Lovelock density of order $n$ is defined by

$$
\mathcal{X}_{2 n}(R) \equiv \frac{1}{2^{n}} \delta_{\nu_{1} \nu_{2} \cdots \nu_{2 n-1} \nu_{2 n}}^{\mu_{1} \mu_{2} \cdots \mu_{2 n-1} \mu_{2 n}} R_{\mu_{1} \mu_{2}}^{\nu_{1} \nu_{2}} \cdots R_{\mu_{2 n-1} \mu_{2 n}}^{\nu_{2 n-1} \nu_{2 n}}
$$

where $\delta_{\nu_{1} \nu_{2} \cdots \nu_{2 n-1} \nu_{2 n}}^{\mu_{1} \mu_{2} \cdots \mu_{2 n-1} \mu_{2 n}}$ is the totally antisymmetric product of $2 n$ Kronecker deltas. Now, since we have that

$$
\left.\frac{\partial R_{\rho \sigma}^{\mu \nu}}{\partial R_{z i z j}}=\frac{1}{2}\left(g^{z[\mu} g^{\nu] i} \delta_{[\rho}^{z} \delta_{\sigma]}^{j}+g^{z[\mu} g^{\nu] j} \delta_{[\rho}^{z} \delta_{\sigma]}^{i}\right)=2 \delta_{\bar{z}}^{[\mu} \delta_{m}^{\nu]} \delta_{[\rho}^{z} \delta_{\sigma]}^{(i}\right]^{j) m},
$$

and a similar result for the derivative with respect to $R_{\bar{z} k \bar{z} l}$, the second derivative contracted with $K^{2}$ appearing in the anomaly term is of the form:

$$
\frac{8 \partial^{2} \mathcal{X}_{2 n}}{\partial R_{z i z j} \partial R_{\bar{z} k \bar{z} l}} K_{z i j} K_{\bar{z} k l}=\frac{-8 n(n-1)}{2^{n-2}} \delta_{z \bar{z} k l \nu_{1} \nu_{2} \ldots \nu_{2 n-5} \nu_{2 n-4}}^{z \bar{z} i j \mu_{1} \mu_{2} \ldots \mu_{2 n-4} \mu_{2 n-4}} R_{\mu_{1} \mu_{2}}^{\nu_{1} \nu_{2}} \cdots R_{\mu_{2 n-5} \nu_{2 n-4}}^{\nu_{2 n-5} \nu_{2 n-4}} K_{z i}{ }^{k} K_{\bar{z} j}{ }^{l}
$$

Due to the completely antisymmetric character of the generalized delta, none of the indices $\mu_{n}$ or $\nu_{n}$ can be $z$ or $\bar{z}$. This forces all components of the Riemann tensor to be of the type $R_{i_{1} i_{2}}^{j_{1} j_{2}}$, as anticipated. ${ }^{10}$ Therefore, we only have to take into account the part proportional to $\partial / \partial R_{i j k l}$ in (3.31). Using the result (3.35) valid when only type $A$ terms are present

\footnotetext{
${ }^{10}$ Something similar happens with the Wald term. As a result, the entanglement entropy functional for Lovelock theories can be written in terms of intrinsic curvatures to the surface - see eq. (4.6) below.
} 
we find

$$
\begin{aligned}
& \sum_{\alpha} \frac{1}{1+q_{\alpha}}\left(\frac{8 \partial^{2} \mathcal{L}_{E}^{\text {Lovelock }}}{\partial \text { Riem }^{2}} K^{2}\right)_{\alpha} \\
& \quad=\sum_{S=0}^{\infty} \frac{1}{(S+1) !}\left(2 K_{a i k} K^{a}{ }_{l j} \frac{\partial}{\partial R_{i j k l}}\right)^{S}\left(\frac{8 \partial^{2} \mathcal{L}_{E}^{\text {Lovelock }}}{\partial \text { Riem }^{2}} K^{2}\right) \\
& \quad=\left[2 K_{a i k} K^{a}{ }_{l j} \frac{\partial}{\partial R_{i j k l}}\right]^{-1}\left[\exp \left(2 K_{a i k} K^{a}{ }_{j l} \frac{\partial}{\partial R_{i j k l}}\right)-1\right]\left(\frac{8 \partial^{2} \mathcal{L}_{E}^{\text {Lovelock }}}{\partial \text { Riem }^{2}} K^{2}\right) .
\end{aligned}
$$

This is a rather suggestive expression. On the other hand, we know that for Lovelock theories the combination of the anomaly and Wald terms must reduce to the so-called Jacobson-Myers (JM) functional - see eq. (4.6) below. Let us see how this works when the anomaly term is written as in eq. (3.40). First of all, notice that the extrinsic curvatures in the second derivative can be written covariantly using the antisymmetry of the generalized delta as

$$
\left(\frac{8 \partial^{2} \mathcal{X}_{2 n}}{\partial \operatorname{Riem}^{2}} K^{2}\right)=\frac{n(1-n)}{2^{n-3}} \delta_{k_{1} l_{1} \ldots k_{n-1} l_{n-1}}^{i_{1} j_{1} \ldots i_{n-1} j_{n-1}} R_{i_{1} j_{1}}^{k_{1} l_{1}} \cdots R_{i_{n-2} j_{n-2}}^{k_{n-2} l_{n-2}} K_{a i_{n-1}}{ }^{k_{n-1}} K_{j_{n-1}}^{a}{ }_{l_{n-1}}^{l_{n-1}},
$$

where we have also reduced the generalized delta eliminating the $z$ and $\bar{z}$ indices. Applying $S$ times the differential operator is now straightforward:

$$
\begin{aligned}
& \sum_{S=0}^{\infty} \frac{1}{(S+1) !}\left(2 K_{a i k} K_{l j}^{a} \frac{\partial}{\partial R_{i j k l}}\right)^{S}\left(\frac{8 \partial^{2} \mathcal{X}_{2 n}}{\partial \mathrm{Riem}^{2}} K^{2}\right) \\
& =-\sum_{S=0}^{n-2} \frac{1}{(S+1) !} \frac{n(n-1) \cdots(n-1-S)}{2^{n-3-S}} \delta_{k_{1} l_{1} \cdots k_{n-1} l_{n-1}}^{i_{1} j_{1} \cdots i_{n-1} j_{n-1}} R_{i_{1} j_{1}}^{k_{1} l_{1}} \cdots R_{i_{n-2-S} j_{n-2-S}}^{k_{n-2-S} l_{n-2}}
\end{aligned}
$$

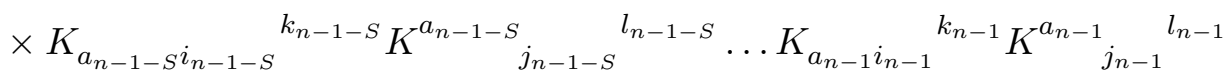

$$
\begin{aligned}
& =-n \sum_{S=1}^{n-1} \frac{1}{2^{n-2-S}}\left(\begin{array}{c}
n-1 \\
S
\end{array}\right) \delta_{k_{1} l_{1} \ldots k_{n-1} l_{n-1}}^{i_{1} j_{1} \ldots i_{n-1} j_{n-1}} R_{i_{1} j_{1}}^{k_{1} l_{1}} \cdots R_{i_{n-1-S} j_{n-1-S}}^{k_{n-1-S} l_{n-1-S}} \\
& \times K_{a_{n-S} i_{n-S}}^{k_{n-S}} K^{a_{n-S}{ }_{j_{n-S}} l_{n-S}} \cdots K_{a_{n-1} i_{n-1}}{ }^{k_{n-1}} K^{a_{n-1}{ }_{j_{n-1}} l_{n-1}} .
\end{aligned}
$$

Furthermore, the Wald term reads

$$
\frac{\partial \mathcal{X}_{2 n}}{\partial R_{z \bar{z} z \bar{z}}}=-\frac{n}{2^{n-2}} \delta_{k_{1} l_{1} \ldots k_{n-1} l_{n-1}}^{i_{1} j_{1} \ldots i_{n-1} j_{n-1}} R_{i_{1} j_{1}}^{k_{1} l_{1}} \ldots R_{i_{n-1} j_{n-1}}^{k_{n-1} l_{n-1}}
$$

This can be combined with (3.42), acting as the $S=0$ term of the sum. When this is included, the binomial coefficient and the $2^{-S}$ factor in each term can be employed to write the full functional as

$$
\begin{gathered}
\frac{\partial \mathcal{X}_{2 n}}{\partial R_{z \bar{z} z \bar{z}}}+\sum_{S=0}^{\infty} \frac{1}{(S+1) !}\left(2 K_{a i k} K^{a}{ }_{l j} \frac{\partial}{\partial R_{i j k l}}\right)^{S}\left(\frac{8 \partial^{2} \mathcal{X}_{2 n}}{\partial \operatorname{Riem}^{2}} K^{2}\right) \\
=-\frac{n}{2^{n-2}} \delta_{k_{1} l_{1} \ldots k_{n-1} l_{n-1}}^{i_{1} j_{1} \ldots i_{n-1} j_{n-1}}\left(R_{i_{1} j_{1}}^{k_{1} l_{1}}+2 K_{a_{1} i_{1}}{ }^{k_{1}} K_{j_{1}}^{a_{1}}{ }^{l_{1}}\right) \\
\quad \ldots\left(R_{i_{n-1} j_{n-1}}^{k_{n-1} l_{n-1}}+2 K_{a_{n-1} i_{n-1}}{ }_{k_{n-1}} K_{j_{n-1}}^{\left.a_{n-1}{ }^{l_{n-1}}\right)}\right.
\end{gathered}
$$


where we used the fact that the binomial factor is the number of ways we can pick $S$ squared extrinsic curvature factors and $(n-1-S)$ Riemann tensors from the previous product (and the antisymmetric delta can be used to rewrite all possible combinations as essentially the same). The final observation is that $\tilde{R}_{i j k l}$ is actually the intrinsic curvature tensor of the surface [32], which we denote $\mathcal{R}_{i j k l}$. Then, comparing with eq. (2.6), it follows that we can write the HEE functional for a given order- $n$ Lovelock density as

$$
S_{\mathrm{HEE}}^{\mathcal{X}_{2 n}}=-4 \pi n \int_{\Gamma_{A}} \mathrm{~d}^{d-1} y \sqrt{h} \mathcal{X}_{2(n-1)}(\mathcal{R}),
$$

which is the JM form $[28,29]$. This has the interesting property of being fully determined in terms of intrinsic curvatures associated to the holographic entangling surface.

\subsection{Anomaly term for cubic gravities}

Our new formula for the anomaly term in (3.30) gets notably simplified for cubic theories. This is a consequence of the second derivative of the Lagrangian being linear in curvatures for these theories, which implies that only $S=0,1$ terms need to be included in the sum. In addition, the object (3.11) is "neutral" in $z$ and $\bar{z}$ indices - i.e., it has and equal number of $z$ 's and $\bar{z}$ 's ${ }^{11}$ - so no components with a different number of $z$ and $\bar{z}$ indices can appear inside it. In particular, there are no type $B$ terms, and the last term appearing in (3.31) is also missing. Therefore, we can write the anomaly term for cubic theories as

$$
\begin{aligned}
\sum_{\alpha} \frac{1}{1+q_{\alpha}}\left(\frac{8 \partial^{2} \mathcal{L}_{E}^{\mathrm{Riem}^{3}}}{\partial \mathrm{Riem}^{2}} K^{2}\right)_{\alpha}= & {\left[1+\frac{1}{4} K^{a i j} K_{a i j} \frac{\partial}{\partial R_{z \bar{z} z \bar{z}}}+4 K_{z i}{ }^{k} K_{\bar{z} j k} \frac{\partial}{\partial R_{z \bar{z} i j}}\right.} \\
& \left.+4 K_{z i}{ }^{k} K_{\bar{z} j k} \frac{\partial}{\partial R_{z i \bar{z} j}}+K_{a i k} K^{a}{ }_{j l} \frac{\partial}{\partial R_{i j k l}}\right]\left(\frac{8 \partial^{2} \mathcal{L}_{E}^{\mathrm{Riem}^{3}}}{\partial \mathrm{Riem}^{2}} K^{2}\right) .
\end{aligned}
$$

In the explicit expressions for the functionals presented in the following section we have obtained the corresponding functionals using both the $\alpha$-expansion procedure and this new derivative expression, finding perfect agreement.

\subsection{Anomaly term for quartic gravities}

Although slightly more complicated than the cubic ones, quartic theories are still simple enough to deserve an independent discussion. In this case, the second derivative of the Lagrangian is quadratic in curvature tensors, so we have to include $S=0,1,2$ in (3.30). However, the neutral character in $z$ 's and $\bar{z}$ 's of (3.11) allows us to simplify the general expression. In the expansion of the second derivative in terms of the basic components of the Riemann tensor, each of the resulting monomials must be neutral in $z$ and $\bar{z}$. The first consequence of this fact is that components $R_{z \bar{z} z \bar{z}}, R_{z \bar{z} i j}, R_{z i \bar{z} j}$, and $R_{i j k l}$ cannot appear paired with the remaining ones, so we can drop all terms that involve mixed second

\footnotetext{
${ }^{11}$ This is a consequence of the scalar character of the Lagrangian, which guarantees that, when written with lower indices, Riemann tensor components are contracted with metrics $g^{\mu \nu}$. The only non-vanishing component in the $z, \bar{z}$ indices is $g^{z \bar{z}}=2$, so for each $z$ there must be one and only one $\bar{z}$. After the two derivatives are taken following (3.11), the number of $z$ 's and $\bar{z}$ 's in Riemann tensor components decreases by two, but it is still equal for both types of indices.
} 
derivatives between these two sets. Furthermore, by the same argument, $R_{z i z j}$ can only appear paired with $R_{\bar{z} i \bar{z} j}$ and thus, at second order in derivatives, type $B$ components do not mix with the type $A$ ones. Also, the last term (in parentheses) in (3.31) does not mix with the remaining part of that operator when taking the square. All this means that the $S=2$ term of (3.30) for quartic theories will be:

$$
\begin{aligned}
& \frac{1}{3 !}\left(\frac{1}{2} K^{a i j} K_{a i j} \frac{\partial}{\partial R_{z \bar{z} z \bar{z}}}+8 K_{z i}{ }^{k} K_{\bar{z} j k} \frac{\partial}{\partial R_{z \bar{z} i j}}+8 K_{z i}{ }^{k} K_{\bar{z} j k} \frac{\partial}{\partial R_{z i \bar{z} j}}+2 K_{a i k} K^{a}{ }_{j l} \frac{\partial}{\partial R_{i j k l}}\right)^{2} \\
& +\frac{1}{3 !}\left(-4 R_{z i z j} \frac{\partial}{\partial R_{z i z j}}+\text { c.c. }\right)^{2}+\frac{2}{4 !}\left(-4 R_{z i j k} \frac{\partial}{\partial R_{z i j k}}-8 R_{z \bar{z} z i} \frac{\partial}{\partial R_{z \bar{z} z i}}+\text { c.c. }\right)^{2}
\end{aligned}
$$

where, although not explicitly written, recall that all derivatives are to be understood under the normal ordering prescription, so they do not act on any of the Riemann tensor components appearing explicitly in the previous expression. This can be simplified a little bit more by using once again the fact that all terms in the second derivative of the Lagrangian have to be neutral in $z$ and $\bar{z}$. Thus, in the second term, only the mixed derivative $\partial^{2} /\left(\partial R_{z i z j} \partial R_{\bar{z} k \bar{z} l}\right)$ contributes. Something similar happens in the last term, where only globally neutral combinations contribute. All in all, including also the $S=0$ and $S=1$ parts of the anomaly term, we can write, for quartic theories:

$$
\begin{aligned}
\sum_{\alpha} & \frac{1}{1+q_{\alpha}}\left(\frac{8 \partial^{2} \mathcal{L}_{E}^{\mathrm{Riem}^{4}}}{\partial \operatorname{Riem}^{2}} K^{2}\right)_{\alpha} \\
= & {\left[1+\frac{1}{2}\left[\frac{1}{2} K^{a i j} K_{a i j} \frac{\partial}{\partial R_{z \bar{z} z \bar{z}}}+8 K_{z i}{ }^{k} K_{\bar{z} j k} \frac{\partial}{\partial R_{z \bar{z} i j}}+8 K_{z i}{ }^{k} K_{\bar{z} j k} \frac{\partial}{\partial R_{z i \bar{z} j}}+2 K_{a i k} K^{a}{ }_{j l} \frac{\partial}{\partial R_{i j k l}}\right.\right.} \\
& \left.\left(-4 R_{z i z j} \frac{\partial}{\partial R_{z i z j}}+\text { c.c. }\right)\right]+\frac{2}{3 !}\left(-4 R_{z i j k} \frac{\partial}{\partial R_{z i j k}}-8 R_{z \bar{z} z i} \frac{\partial}{\partial R_{z \bar{z} z i}}+\text { c.c. }\right) \\
& +\frac{1}{3 !}\left(\frac{1}{2} K^{a i j} K_{a i j} \frac{\partial}{\partial R_{z \bar{z} z \bar{z}}}+8 K_{z i}{ }^{k} K_{\bar{z} j k} \frac{\partial}{\partial R_{z \bar{z} i j}}+8 K_{z i}{ }^{k} K_{\bar{z} j k} \frac{\partial}{\partial R_{z i \bar{z} j}}+2 K_{a i k} K^{a}{ }_{j l} \frac{\partial}{\partial R_{i j k l}}\right)^{2} \\
& +\frac{32}{3 !} R_{z i z j} R_{\bar{z} k \bar{z} l} \frac{\partial^{2}}{\partial R_{z i z j} \partial R_{\bar{z} k \bar{z} l}}+\frac{2}{4 !}\left(128 R_{z \bar{z} z i} R_{\bar{z} z \bar{z} j} \frac{\partial^{2}}{\partial R_{z \bar{z} z i} \partial R_{\bar{z} z \bar{z} j}}\right. \\
& \left.\left.+64 R_{z \bar{z} z i} R_{\bar{z} j k l} \frac{\partial^{2}}{\partial R_{z \bar{z} z i} \partial R_{\bar{z} j k l}}+64 R_{\bar{z} z \bar{z} i} R_{z j k l} \frac{\partial^{2}}{\partial R_{\bar{z} z \bar{z} i} \partial R_{z j k l}}+32 R_{z i j k} R_{\bar{z} l m n} \frac{\partial^{2}}{\partial R_{z i j k} \partial R_{\bar{z} l m n}}\right)\right] \\
& \left(\frac{8 \partial^{2} \mathcal{L}_{E}^{\mathrm{Riem}}{ }^{4}}{\partial \operatorname{Riem}^{2}} K^{2}\right) .
\end{aligned}
$$

When computing the 26 functionals corresponding to independent quartic densities in subsection 4.5 we have made use of this expression, which turns out to be much faster than performing the corresponding $\alpha$ expansions. We have nonetheless verified in a few cases that both procedures yield the same results.

\subsection{An example mixing type $A$ and type $B$ terms}

In the previous subsections involving Lovelock, cubic and quartic densities, we found that it was possible to treat separately type $A$ and $B$ terms. In this subsection we provide a simple example of a situation in which this separation is not possible. The previous arguments show that this happens for densities which are at least fifth-order in the Riemann tensor. 
In order to avoid unnecessary complications, let us assume that one of these densities produces a term mixing type $A$ and $B$ with the following form

$$
\left(\frac{8 \partial^{2} \mathcal{L}_{E}}{\partial \operatorname{Riem}^{2}} K^{2}\right) \supset C\left(K^{2}\right) R_{z i j k} R_{\bar{z} z \bar{z}}^{k} R_{z \bar{z}}^{i j}
$$

where $C\left(K^{2}\right) \equiv c K_{z}{ }^{l m} K_{\bar{z} l m}$ with $c$ a constant, and the $\supset$ symbol means that this is only one of many terms that would appear when expanding the second derivative in terms of the different $z$ and $\bar{z}$ components of the curvature tensor for an actual quintic (or higher order) density. We have not checked whether or not a term like this arises from a concrete fifth order Lagrangian, but it certainly could. ${ }^{12}$ In any case, it will serve as an example of how one should proceed if a different combination of type $A$ and $B$ terms arises.

Let us first obtain the result by means of the $\alpha$ sum, which in this case turns out to be particularly simple. Applying the splitting rules, this term becomes

$$
\sum_{\alpha}\left(\frac{8 \partial^{2} \mathcal{L}_{E}}{\partial \operatorname{Riem}^{2}} K^{2}\right)_{\alpha} \supset C\left(K^{2}\right)\left(R_{z i j k} R_{\bar{z} z \bar{z}}^{k} \tilde{R}_{z \bar{z}}^{i j}-R_{z i j k} R_{\bar{z} z \bar{z}}^{k} K_{z}^{i l} K_{\bar{z} l}^{j}\right) .
$$

The first term has $q_{\alpha}=1$, while the second has $q_{\alpha}=2$. Then, dividing by $1+q_{\alpha}$, we get

$$
\begin{aligned}
\sum_{\alpha} \frac{1}{1+q_{\alpha}}\left(\frac{8 \partial^{2} \mathcal{L}_{E}}{\partial \operatorname{Riem}^{2}} K^{2}\right)_{\alpha} & \supset C\left(K^{2}\right)\left(\frac{1}{2} R_{z i j k} R_{\bar{z} z \bar{z}}^{k} \tilde{R}_{z \bar{z}}^{i j}-\frac{1}{3} R_{z i j k} R_{\bar{z} z \bar{z}}^{k} K_{z}^{i l} K_{\bar{z} l}^{j}\right) \\
= & C\left(K^{2}\right)\left(\frac{1}{2} R_{z i j k} R_{\bar{z} z \bar{z}}{ }^{k} R_{z \bar{z}}^{i j}+\frac{1}{6} R_{z i j k} R_{\bar{z} z \bar{z}}^{k} K_{z}^{i l} K_{\bar{z} l}^{j}\right),
\end{aligned}
$$

where we have rewritten $\tilde{R}_{z \bar{z}}^{i j}$ in terms of the Riemann tensor component again in the last line.

Let us now obtain the same result by means of the derivative expression, (3.30). We need to take into account terms up to $S=3$ in the series, but fortunately not every type $A$ or $B$ component appears in the piece of the Lagrangian we are considering. This means we can define new operators including only the relevant parts:

$$
\partial_{A} \equiv-8 K_{z i}{ }^{l} K_{\bar{z} j l} \frac{\partial}{\partial R_{z i \bar{z} j}}, \quad \partial_{B} \equiv 4 R_{z i j k} \frac{\partial}{\partial R_{z i j k}}+8 R_{\bar{z} z \bar{z} k} \frac{\partial}{\partial R_{\bar{z} z \bar{z} k}} .
$$

Now, the $S=0$ term is just the original (3.49). For the $S=1$ term we apply the operator:

$$
-\frac{1}{2 !} \partial_{A}-\frac{2}{3 !} \partial_{B}
$$

which produces:

$$
s_{1} \equiv C\left(K^{2}\right)\left(-\frac{2}{3} R_{z i j k} R_{\bar{z} z \bar{z}}^{k} R_{z \bar{z}}^{i j}+\frac{1}{2} R_{z i j k} R_{\bar{z} z \bar{z}}^{k} K_{z}^{i l} K_{\bar{z} l}^{j}\right) .
$$

For the $S=2$ term operator we already find mixing between $\partial_{A}$ and $\partial_{B}$. Solving the integral expression given in (3.30)

$$
\frac{1}{2 !} \int_{0}^{1} \mathrm{~d} u 2 u:\left(-\left(1-u^{2}\right) \partial_{A}-(1-u) \partial_{B}\right)^{2}:=:\left(\frac{1}{6} \partial_{A}^{2}+\frac{7}{30} \partial_{A} \partial_{B}+\frac{1}{12} \partial_{B}^{2}\right): .
$$

\footnotetext{
${ }^{12}$ Notice that the term is globally neutral in $z$ and $\bar{z}$, as it should.
} 
We stress once again that normal ordering means that derivatives do not act on curvature components appearing in the operators (3.52), only on those components in the second derivative object (3.49). This makes $\partial_{A}$ and $\partial_{B}$ commuting objects (inside a normal ordered expression). Furthermore, having only a single type $A$ component, the $\partial_{A}^{2}$ term in the previous expression will not contribute. The last two terms produce

$$
s_{2} \equiv C\left(K^{2}\right)\left(\frac{1}{6} R_{z i j k} R_{\bar{z} z \bar{z}}^{k} R_{z \bar{z}}^{i j}-\frac{7}{15} R_{z i j k} R_{\bar{z} z \bar{z}}^{k} K_{z}^{i l} K_{\bar{z} l}^{j}\right) .
$$

Finally, let us consider the $S=3$ term. The operator is

$$
\frac{1}{3 !} \int_{0}^{1} \mathrm{~d} u 2 u:\left(-\left(1-u^{2}\right) \partial_{A}-(1-u) \partial_{B}\right)^{3}:=-:\left(\frac{\partial_{A}^{3}}{24}+\frac{19 \partial_{A}^{2} \partial_{B}}{210}+\frac{\partial_{A} \partial_{B}^{2}}{15}+\frac{\partial_{B}^{3}}{60}\right): .
$$

In this case, since (3.49) has one type $A$ and two type $B$ terms, the third piece of this operator is the only one giving a non-vanishing contribution. Its value is

$$
s_{3} \equiv \frac{2}{15} C\left(K^{2}\right) R_{z i j k} R_{\bar{z} z \bar{z}}^{k} K_{z}^{i l} K_{\bar{z} l}^{j} .
$$

We can finally combine all contributions, $s_{0}$ (which is just the original term (3.49)), $s_{1}, s_{2}$, and $s_{3}$ to obtain

$$
\sum_{\alpha} \frac{1}{1+q_{\alpha}}\left(\frac{8 \partial^{2} \mathcal{L}_{E}}{\partial \operatorname{Riem}^{2}} K^{2}\right)_{\alpha} \supset C\left(K^{2}\right)\left(\frac{1}{2} R_{z i j k} R_{\bar{z} z \bar{z}}^{k} R_{z \bar{z}}^{i j}+\frac{1}{6} R_{z i j k} R_{\bar{z} z \bar{z}}^{k} K_{z}^{i l} K_{\bar{z} l}^{j}\right),
$$

which coincides with (3.51), as it should.

\subsection{Covariant form of the new HEE formula}

So far we have presented all our expressions in the particular set of adapted coordinates $\left(z, \bar{z}, y^{i}\right)$. Here we will rewrite our general formulas in covariant form, which is more useful for explicit applications (like the ones in section 5). In order to do that, we first write the metric as in eq. (1.8),

$$
g_{\mu \nu}=h_{\mu \nu}+\delta_{a b} n_{\mu}^{a} n_{\nu}^{b}
$$

so that in the adapted coordinates $n^{a}{ }_{i}=0$, and $h_{\mu \nu}$ is non-vanishing only for tangent components $\left(h_{z z}=h_{z \bar{z}}=h_{\bar{z} \bar{z}}=0\right)$. It is easy to check that, in the adapted coordinates, the binormal to the surface and the normal projector, defined in eq. (1.11), satisfy $\epsilon_{z \bar{z}}=$ $-\epsilon_{z \bar{z}}=i / 2, \perp_{z z}=\perp_{\bar{z} \bar{z}}=0,{ }^{13}$ and $\perp_{z \bar{z}}=1 / 2$. The following identities can then be shown to hold for the adapted coordinates

$$
\begin{array}{rlrl}
\delta_{\mu}^{z} \delta_{\nu}^{\bar{z}} & =\perp_{\mu \nu}-i \epsilon_{\mu \nu}, & \delta_{z}^{\mu} \delta_{\bar{z}}^{\nu} & =\frac{1}{4}\left(\perp^{\mu \nu}+i \epsilon^{\mu \nu}\right), \\
\delta_{\mu}^{z} \delta_{z}^{\nu}=\frac{1}{2}\left(\perp_{\mu}{ }^{\nu}-i \epsilon_{\mu}{ }^{\nu}\right), & \delta_{\mu}^{\bar{z}} \delta_{\bar{z}}^{\nu} & =\frac{1}{2}\left(\perp_{\mu}{ }^{\nu}+i \epsilon_{\mu}{ }^{\nu}\right)
\end{array}
$$

\footnotetext{
${ }^{13}$ There is an ordering assumption in the value of $\epsilon_{z \bar{z}}$, the normal vectors $n^{1}$ and $n^{2}$ are defined so that we get $\epsilon_{z \bar{z}}=i / 2$.
} 
These are all different forms of the same identity, related by raising or lowering the $z$ and $\bar{z}$ indices, but the different forms are useful in different contexts. In particular, they can be used to write in a covariant form the different terms appearing in the entanglement entropy functional.

Let us start with the Wald term,

$$
\frac{\partial \mathcal{L}_{E}}{\partial R_{z \bar{z} z \bar{z}}}=\delta_{[\mu}^{z} \delta_{\nu]}^{\bar{z}} \delta_{[\rho}^{z} \delta_{\sigma]}^{\bar{z}} \frac{\partial \mathcal{L}_{E}}{\partial R_{\mu \nu \rho \sigma}}=-\epsilon_{\mu \nu} \epsilon_{\rho \sigma} \frac{\partial \mathcal{L}_{E}}{\partial R_{\mu \nu \rho \sigma}} .
$$

The last form, which is the familiar one for this piece [26, 27], is fully covariant, as desired. Similar manipulations can be applied to the anomaly term. For the second derivative of the Lagrangian contracted with two extrinsic curvatures we get ${ }^{14}$

$$
\begin{aligned}
\left(\frac{8 \partial^{2} \mathcal{L}_{E}}{\partial \operatorname{Riem}^{2}} K^{2}\right)= & 2\left[\perp^{\lambda_{1} \lambda_{2}}\left(\perp_{\mu_{1} \mu_{2}} \perp_{\nu_{1} \nu_{2}}-\epsilon_{\mu_{1} \mu_{2}} \epsilon_{\nu_{1} \nu_{2}}\right)+\epsilon^{\lambda_{1} \lambda_{2}}\left(\perp_{\mu_{1} \mu_{2}} \epsilon_{\nu_{1} \nu_{2}}+\epsilon_{\mu_{1} \mu_{2}} \perp_{\nu_{1} \nu_{2}}\right)\right] \\
& \times \frac{\partial^{2} \mathcal{L}_{E}}{\partial R_{\mu_{1} \rho_{1} \nu_{1} \sigma_{1}} \partial R_{\mu_{2} \rho_{2} \nu_{2} \sigma_{2}}} K_{\lambda_{1} \rho_{1} \sigma_{1}} K_{\lambda_{2} \rho_{2} \sigma_{2}} .
\end{aligned}
$$

The operator for the type $A$ terms (3.31) becomes

$$
\begin{aligned}
\mathcal{K}_{A I} \hat{\partial}^{A I}= & {\left[\frac{1}{2} \perp^{\lambda_{1} \lambda_{2}} h^{\tau_{1} \tau_{2}} h^{\omega_{1} \omega_{2}} \epsilon_{\mu \nu} \epsilon_{\rho \sigma}-2 \epsilon^{\lambda_{1} \lambda_{2}} h_{\rho}^{\tau_{1}} h_{\sigma}^{\tau_{2}} h^{\omega_{1} \omega_{2}} \epsilon_{\mu \nu}-2 \perp^{\lambda_{1} \lambda_{2}} h_{\mu}^{\tau_{1}} h_{\rho}^{\omega_{1}} h_{\nu}^{\tau_{2}} h_{\sigma}^{\omega_{2}}\right.} \\
& \left.-2\left(\perp^{\lambda_{1} \lambda_{2}} \perp_{\mu \rho}+\epsilon^{\lambda_{1} \lambda_{2}} \epsilon_{\mu \rho}\right) h_{\nu}^{\tau_{1}} h_{\sigma}^{\tau_{2}} h^{\omega_{1} \omega_{2}}\right] K_{\lambda_{1} \tau_{1} \omega_{1}} K_{\lambda_{2} \tau_{2} \omega_{2}} \frac{\partial}{\partial R_{\mu \nu \rho \sigma}} \\
& +2\left(\perp_{\mu_{2}}^{\mu_{1}} \perp_{\rho_{2}}^{\rho_{1}}-\epsilon_{\mu_{2}}{ }^{\mu_{1}} \epsilon_{\rho_{2}}{ }^{\rho_{1}}\right) h_{\nu_{2}}^{\nu_{1}} h_{\sigma_{2}}^{\sigma_{1}} R_{\mu_{1} \nu_{1} \rho_{1} \sigma_{1}} \frac{\partial}{\partial R_{\mu_{2} \nu_{2} \rho_{2} \sigma_{2}}}
\end{aligned}
$$

while that of type $B$ terms reads

$$
\mathcal{K}_{B I} \hat{\partial}^{B I}=4\left[\perp_{\mu_{2}}^{\mu_{1}} h_{\nu_{2}}^{\nu_{1}} h_{\rho_{2}}^{\rho_{1}} h_{\sigma_{2}}^{\sigma_{1}}+\perp_{\mu_{2}}^{\mu_{1}} \perp_{\nu_{2}}^{\nu_{1}} \perp_{\rho_{2}}^{\rho_{1}} h_{\sigma_{2}}^{\sigma_{1}}\right] R_{\mu_{1} \nu_{1} \rho_{1} \sigma_{1}} \frac{\partial}{\partial R_{\mu_{2} \nu_{2} \rho_{2} \sigma_{2}}} .
$$

Note that, since they always appear in pairs, all the binormals in these expressions could be replaced by normal projectors via the first identity appearing in eq. (1.13), so the whole thing would be written exclusively in terms of contractions of $h_{\mu \nu}$ and $\perp_{\mu \nu}$ with curvature tensors.

The covariant form of the full holographic entanglement entropy functional can be finally written as

$$
\begin{aligned}
S_{\mathrm{HEE}}(A)= & -2 \pi \int_{\Gamma_{A}} d^{D-2} y \sqrt{h}\left[\epsilon_{\mu \nu} \epsilon_{\rho \sigma} \frac{\partial \mathcal{L}_{E}}{\partial R_{\mu \nu \rho \sigma}}\right. \\
& \left.-\sum_{S=0}^{\infty} \frac{1}{S !} \int_{0}^{1} \mathrm{~d} u 2 u:\left(-\left(1-u^{2}\right) \mathcal{K}_{A I} \hat{\partial}^{A I}-(1-u) \mathcal{K}_{B J} \hat{\partial}^{B J}\right)^{S}:\left(\frac{8 \partial^{2} \mathcal{L}_{E}}{\partial \operatorname{Riem}^{2}} K^{2}\right)\right]
\end{aligned}
$$

where derivatives are to be taken respecting the normal ordering prescription introduced in (3.18), and the covariant form of the objects appearing in the last line are given in $(3.63)-(3.65)$.

\footnotetext{
${ }^{14}$ Notice that we take (1.7) as defining a spacetime tensor, $K^{\lambda}{ }_{\mu \nu} \equiv K^{a}{ }_{\mu \nu} n_{a}{ }^{\lambda}$. This tensor satisfies, in adapted coordinates, $K_{\lambda \mu \nu} V^{\nu}=K_{\lambda \mu i} V^{i}$ for any vector $V^{\mu}$.
} 


\section{Explicit covariant form of the functionals}

In this section we present the explicit holographic entanglement entropy functionals for various classes of higher-curvature theories. Like in the rest of the paper, our approach here is to consider such terms as perturbative corrections to Einstein gravity, so that entanglement entropies are computed by the on-shell evaluation of the corresponding Ryu-Takayanagi surfaces on the corrected functionals obtained using the Einstein gravity splitting. We start with a review of the previously known cases of $f(R)$, Lovelock and quadratic theories, for which the splitting problem plays no rôle (and hence the functionals can be also used non-perturbatively). Then, we present new functionals valid for general cubic and quartic theories at leading order in the couplings. We also show that for theories constructed from general contractions of the Ricci tensor and the metric, the anomaly piece vanishes at leading order in the couplings. We observe that the same happens for densities involving a single Riemann tensor, and make general comments on the structure of the perturbative functionals as a function of the number of Riemann tensors.

\section{1 $f(R)$ gravities}

Let us start with $f(R)$ theories. These are the simplest modifications of the Einstein-Hilbert action within the pure-metric class. For an action of the form

$$
I_{E}^{f(R)}=-\frac{1}{16 \pi G} \int \mathrm{d}^{d+1} x \sqrt{|g|}\left[\frac{d(d-1)}{L^{2}}+R+f(R)\right],
$$

the HEE functional only contains a Wald-like piece and is simply given by [32]

$$
S_{\mathrm{HEE}}^{f(R)}=\frac{\mathcal{A}\left(\Gamma_{A}\right)}{4 G}+\frac{1}{4 G} \int_{\Gamma_{A}} \mathrm{~d}^{d-1} y \sqrt{h} f^{\prime}(R) .
$$

Since there is no anomaly piece, this expression can be used non-perturbatively in the putative $f(R)$ couplings by extremizing the full functional.

\subsection{Lovelock gravities}

Let us move to Lovelock theories [47-49]. These are the most general diffemorphisminvariant pure-metric theories of gravity which possess covariantly-conserved second-order equations of motion. The general Euclidean action in $d+1$ dimensions reads

$$
I_{E}^{\text {Lovelock }}=-\frac{1}{16 \pi G} \int \mathrm{d}^{d+1} x \sqrt{|g|}\left[\frac{d(d-1)}{L^{2}}+R+\sum_{n=2}^{\left\lfloor\frac{d+1}{2}\right\rfloor} \lambda_{n} L^{2(n-1)} \mathcal{X}_{2 n}(R)\right],
$$

where $\lfloor x\rfloor$ is the integer part of $x$, the $\lambda_{n}$ are dimensionless couplings and the order- $n$ invariants $\mathcal{X}_{2 n}$ were defined in eq. (3.37) above. $\mathcal{X}_{2 n}$ becomes the Euler density of compact manifolds when evaluated in $2 n$ dimensions. The simplest Lovelock theories (besides Einstein gravity) correspond to the Gauss-Bonnet and cubic densities, which read respectively

$$
\begin{aligned}
\mathcal{X}_{4}= & +R^{2}-4 R_{\mu \nu} R^{\mu \nu}+R_{\mu \nu \rho \sigma} R^{\mu \nu \rho \sigma}, \\
\mathcal{X}_{6}= & +R^{3}-12 R_{\mu}^{\nu} R_{\nu}^{\mu} R+16 R_{\mu}^{\nu} R_{\nu}^{\rho} R_{\rho}^{\mu}+24 R_{\mu \nu \rho \sigma} R^{\mu \rho} R^{\nu \sigma}+3 R R_{\mu \nu \rho \sigma} R^{\mu \nu \rho \sigma} \\
& -24 R_{\mu \nu \rho \sigma} R^{\mu \nu \rho}{ }_{\gamma} R^{\sigma \gamma}-8 R_{\mu \nu}{ }^{\sigma}{ }^{\sigma} R_{\rho}^{\gamma}{ }^{\gamma}{ } R_{\gamma}{ }^{\mu \nu}{ }^{\nu}+4 R_{\mu \nu}{ }^{\rho \sigma} R_{\rho \sigma}{ }^{\gamma \delta} R_{\gamma \delta}{ }^{\mu \nu} .
\end{aligned}
$$


As we mentioned before, for theories beyond quadratic order, the splitting problem challenges the construction of general entanglement entropy functionals. However, the special structure of Lovelock theories makes them unaffected by the splittings choice [4446]. The entanglement entropy is then unambiguously given by the JM functional [28, 29] previously mentioned. This reads, for a general Lovelock theory,

$$
S_{\mathrm{HEE}}^{\text {Lovelock }}=\frac{\mathcal{A}\left(\Gamma_{A}\right)}{4 G}+\sum_{n=2}^{\left\lfloor\frac{d+1}{2}\right\rfloor} \frac{L^{2(n-1)}}{4 G} \int_{\Gamma_{A}} \mathrm{~d}^{d-1} y \sqrt{h} \lambda_{n} \Delta_{n}^{\text {Lovelock }},
$$

where

$$
\Delta_{n}^{\text {Lovelock }}=n \mathcal{X}_{2(n-1)}(\mathcal{R}),
$$

where the lower-order densities are computed with respect to the induced metric $h_{i j}$.

\subsection{Quadratic gravities}

Next we consider theories involving up to four derivatives of the metric. The most general action can be written as

$$
I_{E}^{\mathrm{Riem}^{2}}=-\frac{1}{16 \pi G} \int \mathrm{d}^{d+1} x \sqrt{|g|}\left[\frac{d(d-1)}{L^{2}}+R+L^{2} \sum_{i=1}^{3} \alpha_{i} \mathcal{L}_{i}^{(2)}\right],
$$

where

$$
\mathcal{L}_{1}^{(2)} \equiv R^{2}, \quad \mathcal{L}_{2}^{(2)} \equiv R_{\mu \nu} R^{\mu \nu}, \quad \mathcal{L}_{3}^{(2)} \equiv R_{\mu \nu \rho \sigma} R^{\mu \nu \rho \sigma} .
$$

The HEE functional for this class of theories was first obtained in [30]. It reads

$$
S_{\mathrm{EE}}^{\mathrm{Riem}}{ }^{2}=\frac{\mathcal{A}\left(\Gamma_{A}\right)}{4 G}+\frac{L^{2}}{4 G} \int_{\Gamma_{A}} \mathrm{~d}^{d-1} y \sqrt{h} \sum_{i=1}^{3} \alpha_{i} \Delta_{i}^{(2)},
$$

where

$$
\Delta_{1}^{(2)}=2 R, \quad \Delta_{2}^{(2)}=R_{a}^{a}-\frac{1}{2} K^{a} K_{a}, \quad \Delta_{3}^{(2)}=2\left(R_{a b}^{a b}-K_{a i j} K^{a i j}\right) .
$$

Just like for $f(R)$, and Lovelock theories, there is no splitting problem in this case as the expressions only involve terms quadratic in extrinsic curvatures. Consequently, eq. (4.10) can be trusted at all orders in $\alpha_{i}$. When the terms are considered as perturbative corrections to Einstein gravity, the above expressions get slightly simplified, namely

$$
S_{\mathrm{HEE}}^{\mathrm{Riem}^{2}}=\frac{\mathcal{A}\left(\Gamma_{A}\right)}{4 G}+\frac{L^{2}}{4 G} \int_{\Gamma_{A}} \mathrm{~d}^{d-1} y \sqrt{h} \sum_{i=1}^{3} \alpha_{i} \Delta_{i}^{(2)}+\mathcal{O}\left(\alpha_{i}^{2}\right),
$$

where now

$$
\Delta_{1}^{(2)}=2 R, \quad \Delta_{2}^{(2)}=R_{a}^{a}, \quad \Delta_{3}^{(2)}=2\left(R_{a b}^{a b}-K_{a i j} K^{a i j}\right) .
$$

The difference with respect to the nonperturbative case is the fact that, in this case, the functional that needs to be extremized is the RT one, whose equation of motion reads $K^{a}=0$. We can then remove all the traces of extrinsic curvatures appearing in the highercurvature functionals when looking for expressions valid at leading order in the couplings. 


\subsection{Cubic gravities}

Let us now move to the cubic case. At this order, there are eight independent cubic invariants

$$
I_{E}^{\mathrm{Riem}}{ }^{3}=-\frac{1}{16 \pi G} \int \mathrm{d}^{d+1} x \sqrt{|g|}\left[\frac{d(d-1)}{L^{2}}+R+L^{4} \sum_{i=1}^{8} \beta_{i} \mathcal{L}_{i}^{(3)}\right] .
$$

We label our basis of densities as follows

$$
\begin{aligned}
& \mathcal{L}_{1}^{(3)} \equiv R_{\mu}{ }^{\rho}{ }_{\nu}{ }^{\sigma} R_{\rho \sigma}{ }^{\delta}{ }^{\gamma} R_{\delta}{ }^{\mu}{ }^{\nu}{ }^{\nu}, \quad \mathcal{L}_{2}^{(3)} \equiv R_{\mu \nu}{ }^{\rho \sigma} R_{\rho \sigma}{ }^{\delta \gamma} R_{\delta \gamma}{ }^{\mu \nu}, \\
& \mathcal{L}_{3}^{(3)} \equiv R_{\mu \nu \rho \sigma} R^{\mu \nu \rho}{ }_{\delta} R^{\sigma \delta}, \quad \mathcal{L}_{4}^{(3)} \equiv R_{\mu \nu \rho \sigma} R^{\mu \nu \rho \sigma} R, \\
& \mathcal{L}_{5}^{(3)} \equiv R_{\mu \nu \rho \sigma} R^{\mu \rho} R^{\nu \sigma}, \quad \mathcal{L}_{6}^{(3)} \equiv R_{\mu}^{\nu} R_{\nu}^{\rho} R_{\rho}^{\mu}, \\
& \mathcal{L}_{7}^{(3)} \equiv R_{\mu \nu} R^{\mu \nu} R, \quad \mathcal{L}_{8}^{(3)} \equiv R^{3} .
\end{aligned}
$$

Using our new formula in eq. (3.46) for the anomaly piece, we find the following expression for the functional corresponding to a general cubic Lagrangian of the form (4.14),

$$
S_{\mathrm{HEE}}^{\mathrm{Riem}^{3}}=\frac{\mathcal{A}\left(\Gamma_{A}\right)}{4 G}+\frac{L^{4}}{4 G} \int_{\Gamma_{A}} \mathrm{~d}^{d-1} y \sqrt{h} \sum_{i=1}^{8} \beta_{i} \Delta_{i}^{(3)}+\mathcal{O}\left(\beta_{i}^{2}\right),
$$

where the new terms read

$$
\begin{aligned}
\Delta_{1}^{(3)}= & +\frac{3}{2}\left(R^{a \nu}{ }_{a \mu} R^{b \mu}{ }_{b \nu}-R^{a \nu b \mu} R_{a \mu b \nu}\right) \\
& -\frac{3}{2} R^{i j k l} K_{a i k} K^{a}{ }_{j l}-3 R^{a b i j} K_{a i}{ }^{k} K_{b j k}+\frac{3}{4} R^{a b}{ }_{a b} K^{c i j} K_{c i j}-\frac{3}{8} K^{a i j} K_{a i j} K^{b k l} K_{b k l} \\
& +\frac{9}{4} K_{a i}{ }^{j} K_{b j}{ }^{k} K^{a}{ }_{k}{ }^{l} K^{b}{ }^{i}-\frac{3}{2} K_{a i}{ }^{j} K^{a}{ }_{j}{ }^{k} K_{b k}{ }^{l} K^{b}{ }_{l}{ }^{i}-\frac{3}{4} K_{a i j} K_{b k l} K^{b i j} K^{a k l} \\
\Delta_{2}^{(3)}= & +3 R^{a b \rho \sigma} R_{a b \rho \sigma} \\
& -6 K_{a i}{ }^{k} K_{b j k}\left(R^{a i b j}-R^{b i a j}\right)-6 K_{a i k} K^{a j k} R^{b i}{ }_{b j}+3 K_{a i}{ }^{j} K_{b j}{ }^{k} K^{a}{ }_{k}{ }^{l} K^{b}{ }_{l}{ }^{i} \\
& -6 K_{a i}{ }^{j} K^{a}{ }_{j}{ }^{k} K_{b k}{ }^{l} K^{b}{ }^{i}{ } \\
\Delta_{3}^{(3)}= & +\frac{1}{2} R^{a \mu \nu \rho} R_{a \mu \nu \rho}+2 R^{a \lambda} R^{b}{ }_{a b \lambda} \\
& -K^{a}{ }_{i}{ }^{k} K_{a j k} R^{i j}-\frac{1}{2} K^{a i j} K_{a i j} R_{b}^{b}{ }_{b} \\
\Delta_{4}^{(3)}= & +R_{\mu \nu \rho \sigma} R^{\mu \nu \rho \sigma}+2 R R^{a b}{ }_{a b} \\
& -2 K^{a i j} K_{a i j} R \\
\Delta_{5}^{(3)}= & +R_{\mu}{ }^{\nu} R^{a \mu}{ }_{a \nu}-\frac{1}{2} R^{a b} R_{a b}+\frac{1}{2} R^{a}{ }_{a} R^{b}{ }_{b} \\
\Delta_{6}^{(3)}= & +\frac{3}{2} R^{a \mu} R_{a \mu}, \\
\Delta_{7}^{(3)}= & +R_{\mu \nu} R^{\mu \nu}+R^{a}{ }_{a} R \\
\Delta_{8}^{(3)}= & +3 R^{2} .
\end{aligned}
$$


In each case, the first line corresponds to the Wald-like piece, whereas the rest come from the anomaly one. In the above expressions we have already made use of the RT on-shell condition $K^{a}=0$. If they were to be used nonperturbatively (including extremization of the whole functionals, etc.), additional terms would appear [50]. However, in that case one would need to find first the right splittings in each case and the whole functionals would (most likely) change completely - although the results at $\mathcal{O}\left(\beta_{i}\right)$ will have to reduce to the ones found using the perturbatively valid ones presented here.

We observe that the first two functionals, which are the only ones involving chains of three Riemann tensors, have the most complicated expressions. On the other hand, $\Delta_{3}^{(3)}$ and $\Delta_{4}^{(3)}$, which involve pairs of Riemann tensors are simpler but still have pieces coming from the anomaly part. Finally, densities with a single Riemann or none have a vanishing anomaly piece and their HEE functionals at leading order are just given by the corresponding Wald-entropy expressions. We will see later that this hierarchy in the level of complication of the functionals as a function of the number of Riemann tensors involved actually extends to general-order densities.

Besides the cubic Lovelock densities, there are other interesting theories one can consider and whose HEE functionals can be straightforwardly obtained by replacing the corresponding combinations of $\beta_{i}$ in eq. (4.16). Below, when computing EE universal terms, we will also make explicit the results for a couple of such theories in $d=4$ and $d=3$, respectively. The first is five-dimensional Quasi-topological gravity [51-54], whose action reads

$$
I_{E}^{\mathrm{QTG}}=-\frac{1}{16 \pi G} \int \mathrm{d}^{5} x \sqrt{g}\left[\frac{12}{L^{2}}+R+\frac{7 \mu_{\mathrm{QTG}} L^{4}}{4} \mathcal{Z}_{5}\right]
$$

where

$$
\mathcal{Z}_{5} \equiv \mathcal{L}_{1}^{(3)}-\frac{9}{7} \mathcal{L}_{3}^{(3)}+\frac{3}{8} \mathcal{L}_{4}^{(3)}+\frac{15}{7} \mathcal{L}_{5}^{(3)}+\frac{18}{7} \mathcal{L}_{6}^{(3)}-\frac{33}{14} \mathcal{L}_{7}^{(3)}+\frac{15}{56} \mathcal{L}_{8}^{(3)},
$$

and where we have omitted the usual Gauss-Bonnet density which is usually included in the action. The second is four-dimensional Einsteinian cubic gravity [55-57], whose action is given by

$$
I_{E}^{\mathrm{ECG}}=-\frac{1}{16 \pi G} \int \mathrm{d}^{4} x \sqrt{g}\left[\frac{6}{L^{2}}+R-\frac{\mu_{\mathrm{ECG}} L^{4}}{8} \mathcal{P}\right],
$$

where

$$
\mathcal{P} \equiv 12 \mathcal{L}_{1}^{(3)}+\mathcal{L}_{2}^{(3)}-12 \mathcal{L}_{5}^{(3)}+8 \mathcal{L}_{6}^{(3)}
$$

These theories define holographic toy models of non-supersymmetric CFTs in $d=4$ and $d=3$, respectively. Various holographic aspects of such models have been explored before e.g., in $[8,9,12,13,20,21,58]$. The special properties of Quasi-topological and Einsteinian cubic gravities include the fact that they possess second-order equations on maximally symmetric backgrounds, that they allow for generalizations of the Schwarzschild solution with a single function, i.e., satisfying $g_{t t} g_{r r}=-1$, as well as the fact that the associated thermodynamic properties can be computed fully analytically [59-61]. 


\subsection{Quartic gravities}

At the following order, quartic in curvature, there are 26 independent densities one can write - see e.g., [62, 63],

$$
I_{E}^{\mathrm{Riem}^{4}}=-\frac{1}{16 \pi G} \int \mathrm{d}^{d+1} x \sqrt{|g|}\left[\frac{d(d-1)}{L^{2}}+R+L^{6} \sum_{i=1}^{26} \gamma_{i} \mathcal{L}_{i}^{(4)}\right],
$$

where we choose our basis to be

$$
\begin{aligned}
& \mathcal{L}_{26}^{(4)} \equiv R^{4}, \\
& \mathcal{L}_{25}^{(4)} \equiv R^{2} R_{\mu \nu} R^{\mu \nu}, \\
& \mathcal{L}_{24}^{(4)} \equiv R R_{\mu}{ }^{\nu} R_{\nu}{ }^{\rho} R_{\rho}{ }^{\mu}, \\
& \mathcal{L}_{23}^{(4)} \equiv R_{\mu \nu} R^{\mu \nu} R_{\rho \sigma} R^{\rho \sigma}, \\
& \mathcal{L}_{22}^{(4)} \equiv R_{\mu}{ }^{\nu} R_{\nu}{ }^{\rho} R_{\rho}{ }^{\sigma} R_{\sigma}{ }^{\mu}, \\
& \mathcal{L}_{21}^{(4)} \equiv R R_{\mu \nu \rho \sigma} R^{\mu \rho} R^{\nu \sigma}, \\
& \mathcal{L}_{20}^{(4)} \equiv R^{\mu \nu} R_{\mu \rho \nu \sigma} R^{\delta \rho} R_{\delta}{ }^{\sigma} \text {, } \\
& \mathcal{L}_{19}^{(4)} \equiv R^{2} R_{\mu \nu \rho \sigma} R^{\mu \nu \rho \sigma}, \\
& \mathcal{L}_{18}^{(4)} \equiv R R_{\mu \nu \rho \sigma} R^{\mu \nu \rho}{ }_{\delta} R^{\sigma \delta}, \\
& \mathcal{L}_{17}^{(4)} \equiv R_{\delta \gamma} R^{\delta \gamma} R_{\mu \nu \rho \sigma} R^{\mu \nu \rho \sigma}, \\
& \mathcal{L}_{16}^{(4)} \equiv R^{\mu \nu} R_{\nu}{ }^{\rho} R^{\sigma \delta \gamma}{ }_{\mu} R_{\sigma \delta \gamma \rho}, \\
& \mathcal{L}_{15}^{(4)} \equiv R^{\mu \nu} R^{\rho \sigma} R^{\delta \gamma}{ }_{\mu \rho} R_{\delta \gamma \nu \sigma}, \\
& \mathcal{L}_{14}^{(4)} \equiv R^{\mu \nu} R^{\rho \sigma} R_{\mu}^{\delta}{ }^{\gamma}{ }_{\nu} R_{\delta \rho \gamma \sigma}, \\
& \mathcal{L}_{13}^{(4)} \equiv R^{\mu \nu} R^{\rho \sigma} R_{\mu}^{\delta}{ }_{\rho}{ }_{\rho} R_{\delta \nu \gamma \sigma}, \\
& \mathcal{L}_{12}^{(4)} \equiv R R_{\mu \nu}{ }^{\rho \sigma} R_{\rho \sigma}{ }^{\delta \gamma} R_{\delta \gamma}{ }^{\mu \nu},
\end{aligned}
$$

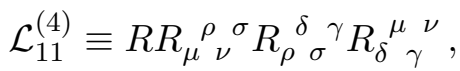

$$
\begin{aligned}
& \mathcal{L}_{10}^{(4)} \equiv R^{\mu \nu} R_{\mu \nu}{ }^{\rho}{ }_{\nu}{ } R_{\delta \gamma \xi \rho} R_{\sigma}^{\delta \gamma \xi}, \\
& \mathcal{L}_{9}^{(4)} \equiv R^{\mu \nu} R^{\rho \sigma \delta \gamma} R_{\rho \sigma}{ }^{\xi}{ }_{\mu} R_{\delta \gamma \xi \nu}, \\
& \mathcal{L}_{8}^{(4)} \equiv R^{\mu \nu} R^{\rho \sigma \delta \gamma} R_{\rho \delta \mu}^{\xi} R_{\sigma \xi \gamma \nu}, \\
& \mathcal{L}_{7}^{(4)} \equiv R_{\mu \nu \rho \sigma} R^{\mu \nu \rho \sigma} R_{\delta \gamma \xi \chi} R^{\delta \gamma \xi \chi}, \\
& \mathcal{L}_{6}^{(4)} \equiv R^{\mu \nu \rho \sigma} R_{\mu \nu \rho}{ }^{\delta} R_{\gamma \xi \chi \sigma} R^{\gamma \xi \chi}{ }_{\delta}, \\
& \mathcal{L}_{5}^{(4)} \equiv R^{\mu \nu \rho \sigma} R_{\mu \nu}{ }^{\delta \gamma} R_{\delta \gamma}{ }^{\chi \xi} R_{\rho \sigma \chi \xi}, \\
& \mathcal{L}_{4}^{(4)} \equiv R^{\mu \nu \rho \sigma} R_{\mu \nu}{ }^{\delta \gamma} R_{\rho \delta}{ }^{\chi \xi} R_{\sigma \gamma \chi \xi}, \\
& \mathcal{L}_{3}^{(4)} \equiv R^{\mu \nu \rho \sigma} R_{\mu \nu}{ }^{\delta \gamma} R_{\rho \delta}^{\chi{ }^{\xi}} R_{\sigma \chi \gamma \xi}, \\
& \mathcal{L}_{2}^{(4)} \equiv R^{\mu \nu \rho \sigma} R_{\mu \rho}^{\delta}{ }^{\gamma} R_{\delta}^{\chi{ }_{\gamma}} R_{\nu \chi \sigma \xi}, \\
& \mathcal{L}_{1}^{(4)} \equiv R^{\mu \nu \rho \sigma} R_{\mu \rho}^{\delta}{ }^{\gamma} R_{\delta}{ }_{\nu}{ }^{\xi} R_{\gamma \chi \sigma \xi} .
\end{aligned}
$$

Using our formula in eq. (3.48), we find the explicit functional for the above densities to be given by

$$
S_{\mathrm{HEE}}^{\mathrm{Riem}^{4}}=\frac{\mathcal{A}\left(\Gamma_{A}\right)}{4 G}+\frac{L^{6}}{4 G} \int_{\Gamma_{A}} \mathrm{~d}^{d-1} y \sqrt{\gamma} \sum_{i=1}^{26} \gamma_{i} \Delta_{i}^{(4)}+\mathcal{O}\left(\gamma_{i}^{2}\right),
$$

where now we find

$$
\begin{aligned}
& \Delta_{26}^{(4)}=+4 R^{3} \\
& \Delta_{25}^{(4)}=+2 R R_{\mu \nu} R^{\mu \nu}+R^{2} R_{a}^{a}, \\
& \Delta_{24}^{(4)}=+R_{\mu}{ }^{\nu} R_{\nu}{ }^{\rho} R_{\rho}{ }^{\mu}+\frac{3}{2} R R^{a \mu} R_{a \mu}, \\
& \Delta_{23}^{(4)}=+2 R_{a}^{a} R_{\mu \nu} R^{\mu \nu}, \\
& \Delta_{22}^{(4)}=+2 R^{a \mu} R_{a}{ }^{\nu} R_{\mu \nu}, \\
& \Delta_{21}^{(4)}=+R_{\mu \nu \rho \sigma} R^{\mu \rho} R^{\nu \sigma}+R R_{a \nu}^{a \mu} R_{\mu}^{\nu}-\frac{1}{2} R R^{a b} R_{a b}+\frac{1}{2} R R_{a}^{a} R_{b}^{b}, \\
& \Delta_{20}^{(4)}=-R_{a \mu \nu \rho} R^{a \rho} R^{\mu \nu}+\frac{1}{2} R_{a \nu}^{a \mu} R_{\mu}{ }^{\rho} R_{\rho}{ }^{\nu}-\frac{1}{2} R_{\mu}{ }^{a} R_{a}{ }^{b} R_{b}{ }^{\mu}+\frac{1}{2} R_{a}^{a}{ }_{a} R_{\mu b} R^{\mu b},
\end{aligned}
$$




$$
\begin{aligned}
& \Delta_{19}^{(4)}=+2 R R_{\mu \nu \rho \sigma} R^{\mu \nu \rho \sigma}+2 R^{2} R_{a b}^{a b} \\
& -2 R^{2} K^{a i j} K_{a i j} \\
& \Delta_{18}^{(4)}=+R_{\mu \nu \rho \sigma} R_{\delta}^{\mu \nu \rho} R^{\sigma \delta}+\frac{1}{2} R R_{a \mu \nu \rho} R^{a \mu \nu \rho}+2 R R_{a \mu}^{a b}{ }_{a \mu}^{\mu} \\
& -\frac{1}{2} R R_{a}^{a} K^{b i j} K_{b i j}-R R^{i j} K_{a i k} K_{j}^{a k} \text {, } \\
& \Delta_{17}^{(4)}=+R_{a}^{a} R_{\mu \nu \rho \sigma} R^{\mu \nu \rho \sigma}+2 R_{a b}^{a b} R_{\mu \nu} R^{\mu \nu} \\
& +2 K^{a i j} K_{a i j}\left(R_{b k} R^{b k}+\frac{2}{3} R_{b c} R^{b c}-R_{\mu \nu} R^{\mu \nu}-\frac{1}{3} R_{b}^{b} R_{c}^{c}\right), \\
& \Delta_{16}^{(4)}=+R_{\mu}{ }^{a} R_{a \nu \rho \sigma} R^{\mu \nu \rho \sigma}+2 R_{\mu}{ }^{a} R_{a b \nu}^{b} R^{\mu \nu} \\
& -\frac{1}{2} K^{a i j} K_{a i j}\left(\frac{1}{2} R_{b k} R^{b k}+\frac{1}{3} R_{b c} R^{b c}+\frac{1}{3} R_{b}^{b} R_{c}^{c}\right)-K_{i}^{a}{ }^{k} K_{a j k}\left(R^{i l} R^{j}{ }_{l}+\frac{1}{2} R^{i b} R^{j}{ }_{b}\right), \\
& \Delta_{15}^{(4)}=+R_{a \mu \rho \sigma} R^{a}{ }_{\nu}{ }^{\rho \sigma} R^{\mu \nu}+2 R^{a \mu} R^{b \nu} R_{a b \mu \nu} \\
& -K_{i}^{a k} K_{a j k}\left(R_{b}^{b} R^{i j}-\frac{1}{2} R^{i b} R_{b}^{j}\right)-K_{i}^{a}{ }^{k} K^{b}{ }_{j k} R^{[i}{ }_{a} R^{j]}{ }_{b}, \\
& \Delta_{14}^{(4)}=+R_{\mu a \rho}^{a} R_{\nu \sigma} R^{\mu \nu \rho \sigma}+R_{a}^{a} R_{\mu b \nu}^{b} R^{\mu \nu}-R_{a \mu b \nu} R^{a b} R^{\mu \nu} \\
& +\frac{1}{24} K^{a i j} K_{a i j}\left(R_{b}^{b} R_{c}^{c}-2 R_{b c} R^{b c}\right)-\frac{1}{4} K^{a}{ }_{i}{ }^{k} K_{a j k} R^{i b} R^{j}{ }_{b}-\frac{1}{2} K^{a}{ }_{i}{ }^{k} K^{b}{ }_{j k} R^{[i}{ }_{a} R^{j]}{ }_{b} \\
& -\frac{1}{2} K^{a}{ }_{i j} K_{a k l} R^{i j} R^{k l}, \\
& \Delta_{13}^{(4)}=+R_{\mu \rho \nu}^{a} R_{a{ }_{\sigma}{ }^{\nu}} R^{\rho \sigma}-R_{a \mu b \nu} R^{a \nu} R^{b \mu}+R_{\mu a \nu}^{a} R^{\mu b} R_{b}^{\nu} \\
& -\frac{1}{8} K^{a i j} K_{a i j} R_{b}^{b} R_{c}^{c}-\frac{1}{2} K^{a}{ }_{i}{ }^{k} K_{a j k} R_{b}^{b} R^{i j}-\frac{1}{2} K^{a}{ }_{i j} K_{a k l} R^{i k} R^{j l}, \\
& \Delta_{12}^{(4)}=+R_{\mu \nu}{ }^{\rho \sigma} R_{\rho \sigma}{ }^{\delta \gamma} R_{\delta \gamma}{ }^{\mu \nu}+3 R R_{a b \mu \nu} R^{a b \mu \nu} \\
& -6 K_{a i}{ }^{k} K_{b j k}\left(R^{a i b j}-R^{b i a j}\right) R-6 K^{a}{ }_{i}^{k} K_{a j k} R^{b i}{ }_{b}^{j} R+3 K_{a i}{ }^{j} K_{b j}{ }^{k} K^{a}{ }_{k}{ }^{l} K^{b}{ }_{l}{ }^{i} R \\
& -6 K_{a i}{ }^{j} K^{a}{ }_{j}{ }^{k} K_{b k}{ }^{l} K^{b i}{ }_{l}^{i} R \text {, } \\
& \Delta_{11}^{(4)}=+R_{\mu \nu}{ }^{\rho}{ }^{\sigma}{ } R_{\rho \sigma}{ }^{\delta}{ }^{\gamma} R_{\delta}{ }^{\mu \nu}{ }^{\nu}+\frac{3}{2} R R_{a \nu}^{a \mu} R_{b \mu}^{b \nu}-\frac{3}{2} R R^{a \mu b \nu} R_{b \mu a \nu} \\
& -\frac{3}{2} K^{a}{ }_{i j} K_{a k l} R^{i k j l} R-3 K_{a i}{ }^{k} K_{b j k} R^{a b i j} R+\frac{3}{4} K^{a i j} K_{a i j} R_{b c}^{b c} R-\frac{3}{8} K^{a i j} K_{a i j} K^{b k l} K_{b k l} R \\
& -\frac{3}{4} K_{a i j} K_{b k l} K^{b i j} K^{a k l} R+\frac{9}{4} K_{a i}{ }^{j} K_{b j}{ }^{k} K^{a}{ }_{k}{ }^{l} K^{b}{ }_{l}^{i} R-\frac{3}{2} K_{a i}{ }^{j} K^{a}{ }_{j}{ }^{k} K_{b k}{ }^{l} K^{b}{ }_{l}^{i} R, \\
& \Delta_{10}^{(4)}=+\frac{1}{2} R_{\mu a \nu}^{a} R_{\rho \sigma \delta}^{\mu} R^{\nu \rho \sigma \delta}+2 R^{\mu \nu} R_{\mu \nu}^{a{ }^{\rho}} R_{a b \rho}^{b}+\frac{1}{2} R_{a}^{a} R_{b \mu \nu \rho} R^{b \mu \nu \rho}-\frac{1}{2} R_{b}^{a} R_{a \mu \nu \rho} R^{b \mu \nu \rho} \\
& +K_{a i j} K_{b k l} R^{i[a} R^{b] k j l}-K_{a i j} K^{a}{ }_{k l}\left(R^{i j} R_{b}{ }^{k b l}-\frac{1}{2} R^{i b} R_{b}{ }^{k j l}\right) \\
& -K_{a i}{ }^{k} K_{b j k} R^{i[a} R_{c}{ }^{b] c j}-\frac{1}{2} K^{a i j} K_{a i j}\left(R^{k l} R_{k b l}^{b}+R^{b k} R_{b c k}^{c}+\frac{1}{2} R_{b}^{b} R^{c d}{ }_{c d}\right) \\
& +K_{a i}{ }^{k} K^{a}{ }_{j k}\left(R_{b l} R^{b i j l}-\frac{1}{2} R_{b}{ }^{i} R_{c}{ }^{b c j}+\frac{2}{3} R_{b c} R^{b i c j}-\frac{1}{6} R_{b}{ }^{b} R_{c}{ }^{i c j}-R_{l m} R^{i l j m}\right) \\
& +\frac{1}{2} K_{a l}{ }^{i} K_{b i}{ }^{j} K^{b}{ }_{j}{ }^{k} K^{a}{ }_{k m} R^{l m}-K_{a i}{ }^{j} K^{a}{ }_{j}{ }^{k} K_{b k}{ }^{i} K^{b}{ }_{l m} R^{l m}-\frac{1}{4} K^{a i j} K_{a i j} K_{b l}{ }^{k} K^{b}{ }_{m k} R^{l m} \\
& +\frac{1}{8} K^{a i j} K_{a i j} K^{b k l} K_{b k l} R_{c}^{c}-\frac{1}{4} K_{a i}{ }^{j} K^{a}{ }_{j}{ }^{k} K_{b k}{ }^{l} K^{b}{ }_{l}^{i} R^{c}{ }_{c},
\end{aligned}
$$




$$
\begin{aligned}
& \Delta_{9}^{(4)}=+\frac{1}{2} R^{\mu \nu \rho \sigma} R_{\mu \nu}^{\xi a} R_{\rho \sigma \xi a}+R^{\mu \nu} R_{\mu}^{a b \rho} R_{a b \rho \nu}-2 R^{\mu a} R_{\mu}^{\nu \rho b} R_{\nu \rho a b} \\
& +2 K_{a i j} K_{b k l} R^{i k} R^{j[a b] l}-K_{a i j} K^{a}{ }_{k l} R^{i k} R_{b}{ }^{j b l}+K_{a i}{ }^{k} K_{b j k}\left(-4 R^{i l} R^{j[a b]}{ }_{l}+R^{i c} R^{a b j}{ }_{c}\right. \\
& \left.-2 R^{a l} R^{b[i j]}{ }_{l}+6 R^{c[a} R^{b] j i}{ }_{c}\right)+K_{a i}{ }^{k} K^{a}{ }_{j k}\left(-2 R^{i l} R_{b l}^{b j}-R_{b}{ }^{i} R_{c}{ }^{b c j}+R_{b l} R^{b i j l}\right. \\
& \left.-\frac{2}{3} R_{b c} R^{b i c j}-\frac{7}{6} R_{b}{ }^{b} R_{c}{ }^{i c j}\right)-\frac{3}{2} K_{a l}{ }^{i} K_{b i}{ }^{j} K^{b}{ }_{j}{ }^{k} K^{a}{ }_{k m} R^{l m}+\frac{3}{2} K_{a l}{ }^{i} K_{b i}{ }^{j} K^{a}{ }_{j}{ }^{k} K^{b}{ }_{k m} R^{l m} \\
& -\frac{3}{2} K_{a l}{ }^{i} K^{a j}{ }_{i} K_{b j}{ }^{k} K^{b}{ }_{k m} R^{l m}+\frac{3}{4} K_{a i}{ }^{j} K_{b j}{ }^{k} K^{a}{ }_{k}{ }^{l} K^{b}{ }_{l}{ }^{i} R^{c}{ }_{c}-\frac{3}{2} K_{a i}{ }^{j} K^{a}{ }_{j}{ }^{k} K_{b k}{ }^{l} K^{b}{ }_{l}^{i} R^{c}{ }_{c} \text {, } \\
& \Delta_{8}^{(4)}=+\frac{1}{2} R^{\mu \nu \rho \sigma} R_{\mu \rho}^{a{ }^{\xi}} R_{\nu a \sigma \xi}+R^{\mu \nu} R_{[a \mid \mu}^{a \rho} R_{\rho \mid b] \nu}^{b}+2 R^{a \mu} R_{\mu[a \mid}{ }^{\rho} R_{\nu \mid b] \rho}^{b} \\
& -\frac{1}{2} K_{a i j} K_{b k l}\left(R^{i[a} R^{b] k j l}+R^{i k} R^{a b j l}\right)+K_{a i j} K_{k l}^{a}\left(R_{m}^{i m} R_{m}^{j k l}-\frac{1}{4} R_{b}^{i} R^{b k j l}-\frac{1}{4} R_{b}^{b} R^{i k j l}\right) \\
& -K_{a i}{ }^{k} K_{b j k}\left(R^{j l} R_{l}^{a b i}-\frac{1}{2} R^{a l} R_{l}^{i j b}+\frac{1}{2} R^{i[a} R_{c}{ }^{b] c j}+\frac{3}{4} R_{c}{ }^{c} R^{a b i j}\right) \\
& +\frac{1}{4} K_{a i}{ }^{k} K_{j k}^{a}\left(R^{i j} R_{b c}^{b c}-R_{b}{ }^{i} R_{c}{ }^{b c j}\right)+\frac{1}{4} K^{a i j} K_{a i j}\left(R^{b k} R_{b c k}^{c}+R_{b}^{b} R^{c d}{ }_{c d}\right) \\
& -\frac{1}{2} K_{a l}{ }^{i} K_{b i}{ }^{j} K^{b}{ }_{j}{ }^{k} K^{a}{ }_{k m} R^{l m}+\frac{5}{4} K_{a l}{ }^{i} K_{b i}{ }^{j} K^{a}{ }_{j}{ }^{k} K^{b}{ }_{k m} R^{l m}-\frac{1}{4} K_{a l}{ }^{i} K^{a}{ }_{i}{ }^{j} K_{b j}{ }^{k} K^{b}{ }_{k m} R^{l m} \\
& -\frac{1}{2} K_{a}{ }^{i j} K_{b i j} K^{a}{ }_{l}^{k} K^{b}{ }_{m k} R^{l m}-\frac{1}{8} K^{a i j} K_{a i j} K_{b l}{ }^{k} K^{b}{ }_{m k} R^{l m}-\frac{1}{8} K_{a i j} K_{b k l} K^{b i j} K^{a k l} R_{c}^{c} \\
& -\frac{1}{8} K^{a i j} K_{a i j} K^{b k l} K_{b k l} R_{c}^{c}+\frac{1}{2} K_{a i}{ }^{j} K_{b j}{ }^{k} K^{a}{ }_{k}{ }^{l} K^{b}{ }_{l}^{i} R^{c}{ }_{c}-\frac{3}{8} K_{a i}{ }^{j} K^{a}{ }_{j}{ }^{k} K_{b k}{ }^{l} K^{b}{ }_{l}^{i} R^{c}{ }_{c}, \\
& \Delta_{7}^{(4)}=+4 R_{\mu \nu \rho \sigma} R^{\mu \nu \rho \sigma} R_{a b}^{a b} \\
& +\frac{64}{3} K_{a i j} K_{b k l} R^{i a j[b \mid} R^{k \mid c] l}{ }_{c}-\frac{8}{3} K_{a i j} K_{k l}^{a} R_{b}^{i b j} R_{c}^{k c l}-4 K^{a i j} K_{a i j}\left(R_{b c}^{b c} R_{d e}^{d e}+2 R^{b c d k} R_{b c d k}\right. \\
& \left.+2 R^{b c k l} R_{b c k l}+\frac{8}{3} R^{b k c l} R_{b k c l}-\frac{4}{3} R^{b k c l} R_{c k b l}+\frac{4}{3} R_{b}{ }^{k b l} R_{k c l}^{c}+2 R^{b k l m} R_{b k l m}+R^{k l m n} R_{k l m n}\right) \\
& -8 K^{a i j} K_{a i j} K_{b k l} K^{b}{ }_{m n} R^{k m l n}-16 K^{a i j} K_{a i j} K_{b k}{ }^{m} K_{c l m}\left(R^{[b|k| c] l}+R^{b c k l}\right) \\
& -8 K^{a i j} K_{a i j} K_{b k}{ }^{m} K^{b}{ }_{l m} R_{c}^{k c l}+4 K^{a i j} K_{a i j} K^{b k l} K_{b k l} R_{c d}^{c d} \\
& +\frac{32}{3} K^{a i j} K_{a i j} K_{b k}{ }^{l} K_{c l}{ }^{m} K^{b}{ }^{n}{ }^{n} K_{n}^{c}{ }^{k}-\frac{32}{3} K^{a i j} K_{a i j} K_{b k}{ }^{l} K^{b}{ }_{l}{ }^{m} K_{c m}{ }^{n} K^{c}{ }^{k}{ }^{k} \\
& -\frac{8}{3} K^{a i j} K_{a i j} K_{b k l} K_{c m n} K^{c k l} K^{b m n}-\frac{4}{3} K^{a i j} K_{a i j} K^{b k l} K_{b k l} K^{c m n} K_{c m n}, \\
& \Delta_{6}^{(4)}=+4 R^{\mu \nu \rho \sigma} R_{\nu \rho \sigma}^{a} R_{a b \mu}^{b} \\
& +2 K_{a i j} K_{b k l}\left(R^{[b \mid i j m} R^{\mid a] k l}+\frac{4}{3} R_{c}^{i a j[b \mid} R_{c}^{k \mid c] l}+2 R^{i k j[a \mid} R^{l c \mid b]}\right) \\
& +K_{a i j} K_{k l}^{a}\left(\frac{1}{3} R_{b}^{i b k} R_{c}^{j c l}-\frac{2}{3} R^{i b k c} R_{b{ }_{c}}^{(j)}-\frac{7}{3} R_{b}^{i b j} R_{c}^{k c l}-R^{i b j m} R_{b m}^{k l}+2 R^{i k j b} R_{b c}^{l c}\right) \\
& +K_{a i}{ }^{k} K_{b j k}\left(R^{i[b \mid c d} R^{j \mid a]}{ }_{c d}+\frac{4}{3} R^{i[b \mid c l} R_{c l}^{j \mid a]}+\frac{4}{3} R^{i[a b] l} R_{c l}^{j c}\right) \\
& +K_{a i}{ }^{k} K^{a}{ }_{j k}\left(-\frac{3}{2} R^{i b c d} R_{b c d}^{j}+2 R_{l[c}^{i b} R_{b]}^{j c l}-3 R^{i b l c} R_{b l c}^{j}-2 R^{i l b c} R^{j}{ }_{l b c}-R^{i b l m} R_{b l m}^{j}\right. \\
& \left.-2 R^{i l b m} R_{l b m}^{j}-2 R^{i l m n} R_{l m n}^{j}\right)+K^{a i j} K_{a i j}\left(-R_{b c}^{b c} R_{d e}^{d e}-\frac{3}{2} R^{b c d k} R_{b c d k}\right. \\
& \left.-R^{b c k l} R_{b c k l}-\frac{4}{3} R^{b k c l} R_{b k c l}+\frac{2}{3} R^{b k c l} R_{c k b l}-\frac{2}{3} R_{b}{ }^{k b l} R_{k c l}^{c}-\frac{1}{2} R^{b k l m} R_{b k l m}\right)
\end{aligned}
$$




$$
\begin{aligned}
& -4 K_{a i}{ }^{k} K_{b k}{ }^{l} K^{b}{ }_{l j} K^{a}{ }_{m n} R^{i m j n}-4 K_{a i}{ }^{k} K_{b k}{ }^{l} K_{c l}{ }^{m} K_{m j}^{c}\left(R^{a b i j}+R^{[a|i| b] j}\right) \\
& -2 K_{a i}{ }^{k} K_{k}^{a}{ }_{k}^{l} K_{b l}{ }^{m} K^{b}{ }_{m j} R_{c}{ }^{i c j}-2 K^{a i j} K_{a i j} K_{b k}{ }^{m} K_{c l m}\left(R^{b c k l}+R^{[b|k| c] l}\right) \\
& -K^{a i j} K_{a i j} K_{b k}{ }^{m} K^{b}{ }_{l m} R_{c}{ }^{k c l}-2 K_{a i}{ }^{j} K^{a}{ }_{j}{ }^{k} K_{b k}{ }^{i} K^{b}{ }_{l m} R_{c}{ }^{l c m}+K^{a i j} K_{a i j} K^{b k l} K_{b k l} R^{c d}{ }_{c d} \\
& +\frac{10}{3} K_{a i}{ }^{j} K^{a}{ }_{j}{ }^{k} K_{b k}{ }^{l} K_{c l}{ }^{m} K^{b}{ }_{m}{ }^{n} K^{c}{ }_{n}{ }^{i}-2 K_{a i}{ }^{j} K^{a}{ }_{j}{ }^{k} K_{b k}{ }^{l} K_{c l}{ }^{m} K^{c}{ }_{m}{ }^{n} K^{b}{ }_{n}{ }^{i} \\
& -\frac{2}{3} K_{a i}{ }^{j} K^{a}{ }_{j}{ }^{k} K_{b k}{ }^{l} K^{b}{ }_{l}{ }^{m} K_{c m}{ }^{n} K^{c}{ }_{n}{ }^{i}-\frac{2}{3} K_{a i}{ }^{j} K^{a}{ }_{j}{ }^{k} K_{b k}{ }^{i} K^{b}{ }_{l}{ }^{m} K_{c m}{ }^{n} K^{c}{ }_{n}{ }^{l} \\
& -\frac{4}{3} K_{a}{ }^{i j} K_{b i j} K_{k}^{a}{ }_{k}^{l} K^{b}{ }_{l}^{m} K_{c m}{ }^{n} K_{n}^{c}{ }^{k}+K^{a i j} K_{a i j} K_{b k}{ }^{l} K_{c l}{ }^{m} K^{b}{ }^{n}{ }^{n} K^{c}{ }_{n}{ }^{k} \\
& -\frac{4}{3} K^{a i j} K_{a i j} K_{b k}{ }^{l} K^{b}{ }_{l}^{m} K_{c m}{ }^{n} K^{c}{ }_{n}{ }^{k}-\frac{1}{3} K^{a i j} K_{a i j} K^{b k l} K_{b k l} K^{c m n} K_{c m n}, \\
& \Delta_{5}^{(4)}=+4 R^{\mu \nu \rho \sigma} R_{\mu \nu}^{a b} R_{\rho \sigma a b} \\
& +16 K_{a i j} K_{b k l} R^{i[a b] k} R_{c}^{j c l}+4 K_{a i j} K_{k l}^{a}\left(2 R^{i b k c} R_{[b c]}^{j l}-R_{b}^{i b k} R_{c}^{j c l}\right) \\
& +8 K_{a i}{ }^{k} K_{b j k}\left(R^{i[b \mid c d} R_{c d}^{j \mid a]}+\frac{4}{3} R^{i[b \mid c l} R^{j \mid a]}{ }_{c l}+\frac{8}{3} R^{i[a b] l} R_{l c}^{j c}+R^{[b \mid i l m} R^{\mid a] j}\right) \\
& -4 K_{a i}{ }^{k} K^{a}{ }_{j k}\left(R^{i b c d} R_{b c d}^{j}+\frac{8}{3} R^{i b l c} R_{b l c}^{j}-\frac{8}{3} R_{[c \mid}^{i b l} R_{l \mid b]}^{j c}+R^{i b l m} R_{b l m}^{j}\right) \\
& +48 K_{a i}{ }^{k} K_{b k}{ }^{l} K_{c l}{ }^{m} K^{c}{ }_{m j} R^{i[a b] j}+24 K_{a i}{ }^{k} K_{c k}{ }^{l} K_{b l}{ }^{m} K^{c}{ }_{m j} R^{a i b j} \\
& -12 K_{a i}{ }^{k} K_{c k}{ }^{l} K_{l}^{c}{ }_{l}^{m} K_{b m j} R^{a i b j}-12 K_{c i}{ }^{k} K_{a k}{ }^{l} K_{b l}{ }^{m} K^{c}{ }_{m j} R^{a i b j} \\
& -12 K_{a i}{ }^{k} K^{a}{ }_{k}{ }^{l} K_{b l}{ }^{m} K^{b}{ }_{m j} R_{c}{ }^{i c j}+12 K_{a i}{ }^{j} K^{a}{ }_{j}{ }^{k} K_{b k}{ }^{l} K_{c l}{ }^{m} K^{b}{ }_{m}{ }^{n} K^{c}{ }_{n}{ }^{i} \\
& -12 K_{a i}{ }^{j} K^{a}{ }_{j}{ }^{k} K_{b k}{ }^{l} K_{c l}{ }^{m} K^{c}{ }_{m}{ }^{n} K^{b}{ }_{n}{ }^{i}-4 K_{a i}{ }^{j} K^{a}{ }_{j}{ }^{k} K_{b k}{ }^{l} K^{b}{ }_{l}{ }^{m} K_{c m}{ }^{n} K^{c}{ }_{n}{ }^{i} \text {, } \\
& \Delta_{4}^{(4)}=+4 R^{a b \mu \nu} R_{a \mu}{ }^{\rho \sigma} R_{b \nu \rho \sigma} \\
& +4 K_{a i j} K_{b k l}\left(2 R^{i[a b] k} R_{c}^{j c l}{ }_{c}-R^{[a \mid i k m} R^{\mid b] l j}\right)+2 K_{a i j} K_{k l}^{a}\left(\frac{4}{3} R^{i b k}{ }_{[c} R^{l c j}{ }_{b]}\right. \\
& \left.-\frac{4}{3} R^{i b k c} R_{b c}^{l j}-R^{i b k m} R_{b m}^{l j}\right)+2 K_{a i}{ }^{k} K_{b j k}\left(-2 R^{a b i j} R_{c d}^{c d}+\frac{4}{3} R^{i[a \mid c l} R^{j \mid b]}{ }_{c l}\right. \\
& \left.+\frac{4}{3} R^{i[a b] l} R_{l c}^{j c}-4 R^{i j a l} R_{l c}^{b c}+R^{[a \mid i l m} R_{l m}^{\mid b] j}-2 R^{a b l m} R_{l m}^{i j}\right) \\
& +K_{a i}{ }^{k} K^{a}{ }_{j k}\left(-2 R^{i b c d} R_{b c d}^{j}-\frac{14}{3} R^{i b l c} R_{b l c}^{j}+\frac{20}{3} R_{[c \mid}^{i b l} R_{l \mid b]}^{j c}-R^{i b l m} R_{b l m}^{j}\right) \\
& -4 K_{a i}{ }^{m} K_{b j m} K_{k}^{a}{ }^{n} K^{b}{ }_{l n} R^{i j k l}+4 K_{a i}{ }^{k} K_{b k}{ }^{l} K_{c l}{ }^{m} K_{m j}^{c}\left(R^{a b i j}+4 R^{i[a b] j}\right) \\
& +4 K_{a i}{ }^{k} K_{c k}{ }^{l} K_{b l}{ }^{m} K_{m j}^{c}\left(R^{a i b j}-R^{a b i j}\right)-2 K_{a i}{ }^{k} K_{c k}{ }^{l} K^{c}{ }_{l}^{m} K_{b m j} R^{a i b j} \\
& -2 K_{c i}{ }^{k} K_{a k}{ }^{l} K_{b l}{ }^{m} K^{c}{ }_{m j} R^{a i b j}-6 K_{a i}{ }^{k} K^{a}{ }_{k}{ }^{l} K_{b l}{ }^{m} K^{b}{ }_{m j} R_{c}{ }^{i c j} \\
& +2 K^{c l m} K_{c l m} K_{a i}{ }^{k} K_{b j k} R^{a b i j}+2 K_{a i}{ }^{j} K_{b j}{ }^{k} K_{k}^{a}{ }_{k}{ }^{l} K^{b}{ }_{l}^{i} R^{c d}{ }_{c d} \\
& -2 K_{a i}{ }^{j} K^{a}{ }_{j}{ }^{k} K_{b k}{ }^{l} K^{b}{ }_{l}^{i} R^{c d}{ }_{c d}+\frac{2}{3} K_{a i}{ }^{j} K^{a}{ }_{j}{ }^{k} K_{b k}{ }^{l} K_{c l}{ }^{m} K^{b}{ }_{m}{ }^{n} K^{c}{ }_{n}{ }^{i} \\
& +2 K_{a i}{ }^{j} K^{a}{ }_{j}{ }^{k} K_{b k}{ }^{l} K_{c l}{ }^{m} K^{c}{ }_{m}{ }^{n} K^{b}{ }_{n}{ }^{i}-\frac{14}{3} K_{a i}{ }^{j} K^{a}{ }_{j}{ }^{k} K_{b k}{ }^{l} K^{b}{ }_{l}{ }^{m} K_{c m}{ }^{n} K^{c}{ }_{n}{ }^{i} \\
& -\frac{4}{3} K^{a i j} K_{a i j} K_{b k}{ }^{l} K_{c l}{ }^{m} K^{b}{ }_{m}{ }^{n} K^{c}{ }_{n}{ }^{k}+\frac{4}{3} K^{a i j} K_{a i j} K_{b k}{ }^{l} K^{b}{ }_{l}{ }^{m} K_{c m}{ }^{n} K_{n}^{c}{ }^{k}, \\
& \Delta_{3}^{(4)}=+2 R^{a b \mu \nu} R_{a \mu}{ }^{\rho}{ }^{\sigma} R_{b \rho \nu \sigma}+R^{\mu a \rho \sigma} R^{\nu}{ }_{a \rho \sigma} R_{\mu b \nu}^{b}-R^{\mu a \rho \sigma} R_{\rho \sigma}^{\nu b} R_{\mu b \nu a} \\
& -2 K_{a i j} K_{b k l}\left(R^{i k j[a \mid} R^{l c \mid b]}+R^{i k a b} R_{c}^{j c l}+2 R^{i[a b] m} R_{m}^{j k l}+R^{[a \mid i k m} R^{j l \mid b]}\right)
\end{aligned}
$$




$$
\begin{aligned}
& +K_{a i j} K_{k l}^{a}\left(2 R^{i k b c} R_{b c}^{j l}-\frac{1}{2} R^{i k b c} R_{b c}^{j l}-R^{i k j b} R_{b c}^{l c}+R^{i k b m} R_{b m}^{j l}-\frac{1}{2} R^{i k b m} R_{b m}^{j l}\right. \\
& \left.+2 R_{b}^{i b m} R_{m}^{j k l}-\frac{1}{2} R^{i k m n} R_{m n}^{j l}\right)-K_{a i}{ }^{k} K_{b j k}\left(R^{i[a b] j} R^{c d}{ }_{c d}+R^{a b i j} R^{c d}{ }_{c d}\right. \\
& \left.+\frac{1}{2} R^{i[a \mid c d} R_{c d}^{j \mid b]}+2 R^{i j a l} R_{l c}^{b c}+R^{[a \mid l i m} R_{m l}^{\mid b]}{ }_{m}^{j}+R^{a b l m} R_{l m}^{i j}+2 R^{a l b m} R_{[l m]}^{i j}\right) \\
& +K_{a i}{ }^{k} K^{a}{ }_{j k}\left(\frac{1}{2} R_{b}{ }^{i b j} R^{c d}{ }_{c d}-\frac{1}{4} R^{i b c d} R_{b c d}^{j}-R^{b}{ }_{l b m} R^{i l j m}+\frac{1}{2} R^{i l b m} R_{m b l}^{j}\right) \\
& +\frac{1}{4} K^{a i j} K_{a i j}\left(R_{b c}^{b c} R_{d e}^{d e}+R^{b c d k} R_{b c d k}+R^{b c k l} R_{b c k l}\right) \\
& +K_{a i}{ }^{m} K_{b j m} K_{k}^{a}{ }^{n} K^{b}{ }_{l n}\left(\frac{1}{2} R^{i l j k}-R^{i j k l}-\frac{3}{2} R^{i k j l}\right)-\frac{1}{2} K_{a i}{ }^{m} K^{a}{ }_{j m} K_{b k}{ }^{n} K^{b}{ }_{l n} R^{i k j l} \\
& +K_{a i j} K_{b k}{ }^{m} K_{m}^{a}{ }^{n} K_{n l}^{b} R^{i k j l}-2 K_{a i j} K_{k}^{a}{ }^{m} K_{b m}{ }^{n} K^{b l}{ }^{b l} R^{i k j l} \\
& -K_{a i}{ }^{k} K_{b k}{ }^{l} K_{c l}{ }^{m} K^{c}{ }_{m j}\left(2 R^{b i a j}+3 R^{a i b j}\right)+K_{a i}{ }^{k} K_{c k}{ }^{l} K_{b l}{ }^{m} K^{c}{ }_{m j}\left(3 R^{a i b j}+R^{b i a j}-2 R^{a b i j}\right) \\
& +\frac{1}{2} K_{a i}{ }^{k} K_{c k}{ }^{l} K_{l}^{c}{ }_{l}^{m} K_{b m j} R^{a i b j}+\frac{1}{2} K_{c i}{ }^{k} K_{a k}{ }^{l} K_{b l}{ }^{m} K^{c}{ }_{m j} R^{a i b j}+K_{a}{ }^{l m} K_{b l m} K_{c i}{ }^{k} K^{c}{ }_{j k} R^{a i b j} \\
& -2 K^{c l m} K_{b l m} K_{a i}{ }^{k} K_{c j k} R^{a i b j}+K^{c l m} K_{c l m} K_{a i}{ }^{k} K_{b j k}\left(R^{a b i j}+\frac{3}{4} R^{b i a j}+\frac{1}{4} R^{a i b j}\right) \\
& +\frac{3}{2} K_{a i}{ }^{k} K^{a}{ }_{k}{ }^{l} K_{b l}{ }^{m} K^{b}{ }_{m j} R_{c}{ }^{i c j}-\frac{1}{2} K_{a k}{ }^{l} K_{b l}{ }^{m} K^{b}{ }_{m}{ }^{k} K^{a}{ }_{i j} R_{c}{ }^{i c j}-\frac{5}{4} K^{b l m} K_{b l m} K_{a i}{ }^{k} K^{a}{ }_{j k} R_{c}{ }^{i c j} \\
& +\frac{1}{4} K_{a i}{ }^{j} K_{b j}{ }^{k} K_{k}^{a}{ }_{k}{ }^{2} K_{l}^{b i} R^{c d}{ }_{c d}-\frac{1}{4} K^{a i j} K_{a i j} K^{b k l} K_{b k l} R^{c d}{ }_{c d} \\
& +\frac{2}{3} K_{a i}{ }^{j} K^{a}{ }_{j}{ }^{k} K_{b k}{ }^{l} K_{c l}{ }^{m} K^{b}{ }^{n}{ }^{n} K^{c}{ }_{n}{ }^{i}+2 K_{a i}{ }^{j} K^{a}{ }_{j}{ }^{k} K_{b k}{ }^{l} K_{c l}{ }^{m} K^{c}{ }_{m}{ }^{n} K^{b}{ }_{n}{ }^{i} \\
& -\frac{4}{3} K_{a i}{ }^{j} K^{a}{ }_{j}{ }^{k} K_{b k}{ }^{l} K^{b}{ }_{l}{ }^{m} K_{c m}{ }^{n} K_{n}^{c}{ }^{i}-\frac{1}{3} K_{a i}{ }^{j} K_{b j}{ }^{k} K_{c k}{ }^{i} K^{a}{ }_{l}{ }^{m} K^{b}{ }_{m}{ }^{n} K_{n}^{c}{ }_{n}{ }^{l} \\
& -\frac{1}{3} K_{a i}{ }^{j} K^{a}{ }_{j}{ }^{k} K_{b k}{ }^{i} K_{c l}{ }^{m} K^{c}{ }_{m}{ }^{n} K^{b}{ }_{n}{ }^{l}-\frac{2}{3} K_{a}{ }^{i j} K_{b i j} K^{a}{ }_{k}{ }^{l} K^{b}{ }_{l}{ }^{m} K_{c m}{ }^{n} K^{c}{ }_{n}{ }^{k} \\
& -\frac{1}{6} K^{a i j} K_{a i j} K_{b k}{ }^{l} K^{b}{ }_{l}^{m} K_{c m}{ }^{n} K_{n}^{c}{ }^{k}+\frac{1}{12} K^{a i j} K_{a i j} K^{b k l} K_{b k l} K^{c m n} K_{c m n}, \\
& \Delta_{2}^{(4)}=+4 R^{\mu \nu \rho \sigma} R_{\mu a \rho}^{[a} R_{\nu b \sigma}^{b]} \\
& +2 K_{a i j} K_{b k l}\left(\frac{2}{3} R^{i a j[c \mid} R_{c}^{k \mid b] l}+2 R^{i k j[a \mid} R_{c}^{l c \mid b]}-R^{i[a b] k} R^{j c l}\right) \\
& +K_{a i j} K_{k l}^{a}\left(R^{i k j l} R_{b c}^{b c}-2 R^{i k j b} R_{b c}^{l c}-\frac{1}{6} R_{b}^{i b j} R_{c}^{k c l}-\frac{1}{2} R_{b}^{i b k} R_{c}^{j c l}-\frac{4}{3} R^{i b j c} R_{b c}^{k l}\right. \\
& \left.+R^{i b k c} R_{b{ }_{c}}^{[j l]}+R^{i k b c} R_{b c}^{j l}-2 R^{i b j m} R_{b m}^{k l}-2 R^{i m j n} R_{m n}^{k l}\right) \\
& -2 K_{a i}{ }^{k} K_{b j k}\left(\frac{8}{3} R^{i[a b] l} R_{l c}^{j c}+\frac{2}{3} R^{i[a \mid c l} R^{j \mid b]}+R^{[a \mid l i m} R_{l m}^{\mid b]}\right) \\
& -K_{a i}{ }^{k} K^{a}{ }_{j k}\left(R^{i b c d} R_{b c d}^{j}+\frac{4}{3} R^{i b l c} R_{b l c}^{j}+\frac{4}{3} R_{[b \mid}^{i b l} R_{l \mid c]}^{j c}+2 R^{i l b c} R_{l b c}^{j}+R^{i l b m} R_{l b m}^{j}\right) \\
& -\frac{1}{2} K^{a i j} K_{a i j}\left(R_{b c}^{b c} R_{d e}^{d e}+R^{b c d k} R_{b c d k}+\frac{2}{3} R^{b k c l} R_{(b|k| c) l}-\frac{1}{3} R_{b}^{k b l} R_{k c l}^{c}\right) \\
& +2 K_{a i j} K_{b k}{ }^{m} K_{m}^{a}{ }^{n} K^{b}{ }_{n l} R^{i k j l}-2 K_{a}{ }^{m n} K_{b m n} K^{a}{ }_{i j} K^{b}{ }_{k l} R^{i k j l}-\frac{1}{2} K^{a m n} K_{a m n} K_{b i j} K_{k l}^{b} R^{i k j l} \\
& -2 K_{a i}{ }^{k} K_{b k}{ }^{l} K_{c l}{ }^{m} K^{c}{ }_{m j}\left(3 R^{a b i j}+R^{i(a b) j}\right)+K_{a i}{ }^{k} K_{c k}{ }^{l} K_{b l}{ }^{m} K^{c}{ }_{m j}\left(2 R^{a b i j}-R^{a i b j}-2 R^{b i a j}\right) \\
& +\frac{1}{2} K_{a i}{ }^{k} K_{c k}{ }^{l} K^{c}{ }_{l}^{m} K_{b m j} R^{a i b j}+\frac{1}{2} K_{c i}{ }^{k} K_{a k}{ }^{l} K_{b l}{ }^{m} K_{m j}^{c} R^{a i b j}-\frac{3}{2} K_{a i}{ }^{k} K_{k}^{a}{ }_{k}{ }^{l} K_{b l}{ }^{m} K^{b}{ }_{m j} R_{c}{ }^{i c j}
\end{aligned}
$$




$$
\begin{aligned}
& -K_{a k}{ }^{l} K_{b l}{ }^{m} K^{b}{ }_{m}{ }^{k} K^{a}{ }_{i j} R_{c}{ }^{i c j}-\frac{1}{2} K_{a i}{ }^{j} K_{b j}{ }^{k} K^{a}{ }_{k}{ }^{l} K^{b}{ }_{l}^{i} R^{c d}{ }_{c d}+\frac{1}{2} K_{a}{ }^{i j} K_{b i j} K^{a k l} K^{b}{ }_{k l} R^{c d}{ }_{c d} \\
& +\frac{1}{2} K^{a i j} K_{a i j} K^{b k l} K_{b k l} R^{c d}{ }_{c d}+\frac{5}{6} K_{a i}{ }^{j} K^{a}{ }_{j}{ }^{k} K_{b k}{ }^{l} K_{c l}{ }^{m} K^{b}{ }_{m}{ }^{n} K^{c}{ }_{n}{ }^{i} \\
& -\frac{7}{2} K_{a i}{ }^{j} K^{a}{ }_{j}{ }^{k} K_{b k}{ }^{l} K_{c l}{ }^{m} K^{c}{ }_{m}{ }^{n} K^{b}{ }_{n}{ }^{i}+\frac{3}{2} K_{a i}{ }^{j} K^{a}{ }_{j}{ }^{k} K_{b k}{ }^{l} K^{b}{ }_{l}{ }^{m} K_{c m}{ }^{n} K^{c}{ }_{n}{ }^{i} \\
& -\frac{1}{3} K_{a i}{ }^{j} K^{a}{ }_{j}{ }^{k} K_{b k}{ }^{i} K_{c l}{ }^{m} K_{m}^{c}{ }^{n} K^{b}{ }_{n}{ }^{l}+\frac{4}{3} K_{a}{ }^{i j} K_{b i j} K^{a}{ }_{k}{ }^{l} K^{b}{ }_{l}{ }^{m} K_{c m}{ }^{n} K^{c}{ }_{n}{ }^{k} \\
& +K^{a i j} K_{a i j} K_{b k}{ }^{l} K_{c l}{ }^{m} K^{b}{ }_{m}{ }^{n} K_{n}^{c}{ }^{k}-\frac{2}{3} K^{a i j} K_{a i j} K_{b k}{ }^{l} K^{b}{ }_{l}^{m} K_{c m}{ }^{n} K^{c}{ }_{n}{ }^{k} \\
& -\frac{4}{3} K^{a i j} K_{a i j} K_{b}{ }^{k l} K_{c k l} K^{b m n} K_{m n}^{c}+\frac{1}{6} K^{a i j} K_{a i j} K^{b k l} K_{b k l} K^{c m n} K_{c m n}, \\
& \Delta_{1}^{(4)}=+4 R^{a \mu \rho \nu} R_{\nu[a \mid \mu}^{\sigma} R_{\rho \mid b] \sigma}^{b} \\
& +K_{a i j} K_{b k l}\left(\frac{4}{3} R^{i a j[c \mid} R_{c}^{k \mid b] l}+2 R^{i k j[a \mid} R_{c}^{l c \mid b]}+4 R^{i[a b] m} R_{m}^{j k l}+R^{[a \mid i j m} R^{\mid b] k l}\right) \\
& +K_{a i j} K_{k l}^{a}\left(-R^{i k j b} R_{b c}^{l c}-\frac{1}{3} R_{b}^{i b k} R_{c}^{j c l}-\frac{2}{3} R^{i b j c} R_{b c}^{k l}-\frac{2}{3} R^{i b k c} R_{b c}^{j l}+\frac{1}{3} R^{i b k c} R_{b c}^{l j}\right. \\
& \left.+R^{i k b c} R_{b c}^{j l}+2 R_{b}^{i b m} R_{m}^{j k l}-\frac{1}{2} R^{i b j m} R_{b m}^{k l}-R^{i b k m} R_{b m}^{j l}+\frac{1}{2} R^{i k b m} R_{b m}^{j l}-R^{i m k n} R_{m n}^{j l}\right) \\
& +K_{a i}{ }^{k} K_{b j k}\left(3 R^{i[a b] j} R_{c d}^{c d}+\frac{1}{2} R^{i[a \mid c d} R^{j \mid b]}{ }_{c d}+\frac{2}{3} R^{i[a b] l} R_{l c}^{j c}-4 R^{i[a \mid j l} R^{\mid b] c}{ }_{l c}+\frac{2}{3} R^{i[a \mid c l} R^{j \mid b]}\right. \\
& \left.-2 R^{a l b m} R_{[l m]}^{i j}\right)+K_{a i}{ }^{k} K^{a}{ }_{j k}\left(\frac{1}{2} R_{b}{ }^{i b j} R_{c d}^{c d}-\frac{3}{4} R^{i b c d} R_{b c d}^{j}+\frac{1}{6} R_{b}^{i b l} R_{l c}^{j c}+\frac{1}{3} R^{i b l c} R_{(b c) l}^{j}\right. \\
& \left.-R^{i l b c} R_{l b c}^{j}-R^{i l j m} R_{l b m}^{b}\right)+\frac{1}{4} K^{a i j} K_{a i j}\left(2 R^{b k c l} R_{[b|k| c] l}-R_{b}^{k b l} R_{k c l}^{c}\right) \\
& +K_{a i}{ }^{m} K_{b j m} K_{k}^{a}{ }^{n} K^{b}{ }_{l n} R^{i(k l) j}-\frac{1}{2} K_{a i}{ }^{m} K^{a}{ }_{j m} K_{b k}{ }^{n} K^{b}{ }_{l n} R^{i k j l}-2 K_{a i j} K_{b k}{ }^{m} K^{a}{ }_{m}{ }^{n} K^{b}{ }_{n l} R^{i k j l} \\
& -2 K_{a i}{ }^{k} K_{b k}{ }^{l} K_{c l}{ }^{m} K^{c}{ }_{m j}\left(2 R^{a b i j}+R^{b i a j}\right)+K_{a i}{ }^{k} K_{c k}{ }^{l} K_{b l}{ }^{m} K^{c}{ }_{m j}\left(2 R^{a b i j}+3 R^{b i a j}-R^{a i b j}\right) \\
& +K_{a}{ }^{l m} K_{b l m} K_{c i}{ }^{k} K^{c}{ }_{j k} R^{a i b j}-2 K_{a}{ }^{l m} K_{c l m} K_{b i}{ }^{k} K^{c}{ }_{j k} R^{b i a j} \\
& +\frac{1}{2} K^{c l m} K_{c l m} K_{a i}{ }^{k} K_{b j k}\left(5 R^{a i b j}-3 R^{b i a j}\right)+K_{a i}{ }^{k} K_{k}^{a}{ }^{l} K_{b l}{ }^{m} K^{b}{ }_{m j} R_{c}{ }^{i c j} \\
& -K_{a k}{ }^{l} K_{b l}{ }^{m} K^{b}{ }_{m}{ }^{k} K^{a}{ }_{i j} R_{c}{ }^{i c j}-\frac{3}{2} K^{b l m} K_{b l m} K_{a i}{ }^{k} K^{a}{ }_{j k} R_{c}{ }^{i c j}+\frac{3}{4} K_{a i}{ }^{j} K_{b j}{ }^{k} K^{a}{ }_{k}{ }^{l} K^{b}{ }_{l}{ }^{i} R^{c d}{ }_{c d} \\
& -\frac{1}{2} K_{a i}{ }^{j} K^{a}{ }_{j}{ }^{k} K_{b k}{ }^{l} K^{b}{ }_{l}^{i} R^{c d}{ }_{c d}+\frac{13}{6} K_{a i}{ }^{j} K^{a}{ }_{j}{ }^{k} K_{b k}{ }^{l} K_{c l}{ }^{m} K^{b}{ }_{m}{ }^{n} K^{c}{ }_{n}{ }^{i} \\
& +\frac{1}{2} K_{a i}{ }^{j} K^{a}{ }_{j}{ }^{k} K_{b k}{ }^{l} K_{c l}{ }^{m} K^{c}{ }_{m}{ }^{n} K^{b}{ }_{n}{ }^{i}-\frac{7}{6} K_{a i}{ }^{j} K^{a}{ }_{j}{ }^{k} K_{b k}{ }^{l} K^{b}{ }_{l}{ }^{m} K_{c m}{ }^{n} K^{c}{ }_{n}{ }^{i} \\
& -\frac{1}{3} K_{a i}{ }^{j} K_{b j}{ }^{k} K_{c k}{ }^{i} K^{a}{ }_{l}{ }^{m} K^{b}{ }_{m}{ }^{n} K^{c}{ }_{n}{ }^{l}-\frac{1}{2} K_{a i}{ }^{j} K^{a}{ }_{j}{ }^{k} K_{b k}{ }^{i} K_{c l}{ }^{m} K^{c}{ }_{m}{ }^{n} K^{b}{ }_{n}{ }^{l} \\
& -K_{a}{ }^{i j} K_{b i j} K_{k}^{a}{ }^{l} K^{b}{ }_{l}^{m} K_{c m}{ }^{n} K^{c}{ }_{n}{ }^{k}-\frac{13}{12} K^{a i j} K_{a i j} K_{b k}{ }^{l} K_{c l}{ }^{m} K^{b}{ }^{n}{ }^{n} K^{c}{ }^{k} \\
& +\frac{5}{6} K^{a i j} K_{a i j} K_{b k}^{l} K^{b}{ }^{m} K_{c m}{ }^{n} K_{n}^{c k} \text {. }
\end{aligned}
$$

Again, we observe that the greater the number of Riemann tensors involved in the corresponding density, the more complicated the expressions. In particular, for theories with zero or one Riemann tensors, the contribution comes completely from the Wald piece. For densities with two Riemanns we get contributions which are quadratic in extrinsic curvatures, for those with three Riemanns, we get terms which are quartic, and for densities with four Riemann tensors there are terms involving up to six extrinsic curvatures. 


\section{6 $\mathcal{L}\left(g_{\mu \nu}, R_{\rho \sigma}\right)$ gravities}

Let us now consider densities constructed from general contractions of the Ricci tensor, i.e., of the form

$$
I_{E}^{\mathcal{L}(\text { Ricci })}=-\frac{1}{16 \pi G} \int \mathrm{d}^{d+1} x \sqrt{|g|}\left[\frac{d(d-1)}{L^{2}}+R+\lambda \mathcal{L}\left(g_{\mu \nu}, R_{\rho \sigma}\right)\right],
$$

where $\lambda$ is some constant. By looking at the quadratic, cubic and quartic densities of this kind, we observe that no contribution from the anomaly part arises in the HEE functional when those terms are considered perturbatively. As we show now, this is in fact a general property which holds for all theories of the form (4.58).

The proof goes as follows. For the anomaly term, we need to compute the second derivative of the Lagrangian with respect to $R_{z i z j}$ and $R_{\bar{z} k \bar{z} l}$. Let us consider first the one with $R_{z i z j}$. Since the Lagrangian is a contraction of $n$ Ricci tensors for an $n$-th order theory, we can expand the derivative as

$$
\frac{\partial \mathcal{L}}{\partial R_{z i z j}}=\sum_{k=1}^{n} \frac{\partial R_{\mu \nu}}{\partial R_{z i z j}} T_{(k)}^{\mu \nu},
$$

where $T_{(k)}^{\mu \nu}$ represents the remaining part of the Lagrangian contracted with each of the Ricci tensors - this can include metric tensors, so the previous expansion is also valid when there are Ricci scalars in the Lagrangian. Now, it can be shown from eq. (3.1) that

$$
\frac{\partial R_{\mu \nu}}{\partial R_{\alpha \beta \gamma \delta}}=\delta_{(\mu}^{[\beta} g^{\alpha][\gamma} \delta_{\nu)}^{\delta]} \Rightarrow \frac{\partial R_{\mu \nu}}{\partial R_{z i z j}}=\frac{1}{4} h^{i j} \delta_{\mu}^{z} \delta_{\nu}^{z},
$$

since $g^{z z}=g^{z i}=0$. Therefore, (4.59) is proportional to $h^{i j}$. An analogous argument with the other derivative shows that it is proportional to $h^{k l}$. The conclusion is that the anomaly term is then some expression containing curvature tensors in which we have to perform the $\alpha$-expansion, times the following contraction of extrinsic curvatures:

$$
h^{i j} h^{k l} K_{z i j} K_{\bar{z} k l}=K_{z} K_{\bar{z}}=\frac{1}{4} K^{a} K_{a},
$$

which vanishes when evaluated for the RT surface. Hence, the anomaly part of the functional does not contribute perturbatively for theories constructed from general contractions of the Ricci tensor. Note that this is actually true irrespective of the splitting being used.

Hence, for theories of this kind one finds

$$
S_{\mathrm{HEE}}^{\mathcal{L}(\mathrm{Ricci})}=\frac{\mathcal{A}\left(\Gamma_{A}\right)}{4 G}+\frac{\lambda}{8 G} \int_{\Gamma_{A}} \mathrm{~d}^{d-1} y \sqrt{h} \frac{\partial \mathcal{L}}{\partial R_{\mu \nu}} \perp_{\mu \nu}+\mathcal{O}\left(\lambda^{2}\right)
$$

We emphasize that this formula holds for general-order densities of the form $\mathcal{L}\left(g_{\mu \nu}, R_{\rho \sigma}\right)$. Hence, we observe that, at least perturbatively in the higher-curvature couplings, the purely-Wald nature of the $f(R)$ functional actually extends to the much greater family of densities constructed from arbitrary contractions of the Ricci tensor and the metric. 


\subsection{General structure depending on the number of Riemann tensors}

The observations made in the previous subsections suggest a more general pattern which we explore here. The starting point is the observation made in subsection 4.6 that whenever one of the two derivatives appearing in (3.11) hits a Ricci tensor, the contraction of the resulting intrinsic metric with the extrinsic curvature produces a trace, $K_{z}$ or $K_{\bar{z}}$, which is zero for the RT surface (and therefore also for the perturbative functional). Consider then an $n$-th order curvature density containing $n_{R}$ Riemann tensors and $n-n_{R}$ Ricci tensors or scalars. After the two derivatives are taken, the only non-vanishing pieces will be of the form

$$
\left(\frac{8 \partial^{2} \mathcal{L}_{E}}{\partial \operatorname{Riem}^{2}} K^{2}\right) \sim \sum K^{2} \operatorname{Ricci}_{1} \ldots \operatorname{Ricci}_{n-n_{R}} \operatorname{Riem}_{1} \ldots \operatorname{Riem}_{n_{R}-2}
$$

In this expression, we use the symbol $\sim$ to represent the structure of the object in terms of the curvature tensors appearing, ignoring the particular components. The sum means that several terms with this structure will show up in general. Each Ricci ${ }_{k}$ represents a particular component of the Ricci tensor or scalar and, analogously, Riem $k$ represents a component of the Riemann tensor.

Observe now the following property. Writing explicitly the Ricci tensor and scalar in terms of Riemann tensor components, we get

$$
\begin{array}{rlrl}
R_{z z} & =h^{i j} R_{z i z j}, & R_{z \bar{z}} & =-2 R_{z \bar{z} z \bar{z}}+h^{i j} R_{z i \bar{z} j}, \\
R_{z i} & =-2 R_{z \bar{z} z i}+h^{j k} R_{z j i k}, & R_{i j}=2 R_{z i \bar{z} j}+2 R_{z j \bar{z} i}+h^{k l} R_{i k j l}, \\
R & =4 R_{z \bar{z}}+h^{i j} R_{i j}, & &
\end{array}
$$

plus the ones obtained by complex conjugation. Then, the differential operators defined in eq. (3.31) and eq. (3.32) act on these components as follows

$$
\begin{aligned}
\mathcal{K}_{A I} \hat{\partial}^{A I} R_{z z} & =R_{z z}, & \mathcal{K}_{B I} \hat{\partial}^{B I} R_{z z} & =0, \\
\mathcal{K}_{A I} \hat{\partial}^{A I} R_{z \bar{z}} & =0, & \mathcal{K}_{B I} \hat{\partial}^{B I} R_{z \bar{z}} & =0, \\
\mathcal{K}_{A I} \hat{\partial}^{A I} R_{z i} & =0, & \mathcal{K}_{B I} \hat{\partial}^{B I} R_{z i} & =R_{z i}, \\
\mathcal{K}_{A I} \hat{\partial}^{A I} R_{i j} & =-K_{a i j} K^{a}, & \mathcal{K}_{B I} \hat{\partial}^{B I} R_{i j} & =0, \\
\mathcal{K}_{A I} \hat{\partial}^{A I} R & =-K_{a} K^{a}, & \mathcal{K}_{B I} \hat{\partial}^{B I} R & =0 .
\end{aligned}
$$

Notice also that if the Ricci components are acted upon with several powers of the differential operators in normal order, like in the functional (3.30), the remaining powers would not act on the curvature tensors appearing in the right-hand side of the previous expressions. In any case, the relevant observation is that after applying the differential operator, any Ricci factor in (4.63) generates either something proportional to the very same component or something proportional to $K^{a}$. When evaluated at the RT surface, this second possibility gives zero, so in a perturbative functional no Ricci tensor component can ever generate powers of the extrinsic curvature. This is not the case with Riemann tensor components, for which the differential operator generates non-vanishing contractions of extrinsic curva- 
tures in general. ${ }^{15}$ The conclusion is that the expression which results from applying the full differential operator of the anomaly term to a second derivative of the form (4.63) has the structure

$$
\begin{aligned}
\sum_{\alpha} & \frac{1}{1+q_{\alpha}}\left(\frac{8 \partial^{2} \mathcal{L}_{E}}{\partial \operatorname{Riem}^{2}} K^{2}\right)_{\alpha} \\
& \sim \sum \operatorname{Ricci}^{n-n_{R}}\left(\operatorname{Riem}^{n_{R}-2} K^{2}+\operatorname{Riem}^{n_{R}-3} K^{4}+\cdots+\operatorname{Riem} K^{2 n_{R}-4}+K^{2 n_{R}-2}\right) .
\end{aligned}
$$

One can verify that this is indeed the case for all quadratic, cubic, and quartic Lagrangian densities presented in the previous sections.

In summary, we have shown that densities containing $n_{R}$ Riemann curvatures can contain terms involving extrinsic curvatures up to the power $2 n_{R}-2$. In particular, this implies that densities with zero or one Riemann tensors have no anomaly piece. We already studied the former case in the previous subsection. As for the latter, for a theory of the form

$$
-\frac{1}{16 \pi G} \int \mathrm{d}^{d+1} x \sqrt{|g|}\left[\frac{d(d-1)}{L^{2}}+R+\lambda R^{\mu \nu \rho \sigma} T_{\mu \nu \rho \sigma}(\text { Ricci })\right],
$$

where $T_{\mu \nu \rho \sigma}($ Ricci) is some tensorial structure involving Ricci tensors and metrics, the corresponding functional reads

$$
\frac{\mathcal{A}\left(\Gamma_{A}\right)}{4 G}+\frac{\lambda}{8 G} \int_{\Gamma_{A}} \mathrm{~d}^{d-1} y \sqrt{h}\left[2 T^{\mu \nu \rho \sigma} \perp_{\mu[\rho} \perp_{\nu \mid \sigma]}+R^{\mu \nu \rho \sigma} \frac{\partial T_{\mu \nu \rho \sigma}}{\partial R_{\alpha \beta}} \perp_{\alpha \beta}\right]+\mathcal{O}\left(\lambda^{2}\right) .
$$

On the other hand, densities with two Riemann tensors have terms with up to two extrinsic curvatures, those with three have terms with up to four extrinsic curvatures, and so on.

\section{Universal terms}

In this section we study how the universal coefficients appearing in the EE of various symmetric entangling regions get modified in the presence of quadratic and cubic corrections. Some of these coefficients can be computed from alternative methods, and in that case we verify that the results agree with them. In other cases, like for strip regions, the corresponding universal coefficients do not have a known alternative interpretation beyond entanglement entropy. Universal terms for various types of regions have been previously computed for particular higher-curvature theories in certain dimensions in several papers such as $[7,8,28,36,45,64-67]$. Our results reproduce the ones found in those papers in the appropriate cases.

We will restrict ourselves to the vacuum state. This means that all expressions involving intrinsic bulk curvatures will be evaluated on pure $\mathrm{AdS}_{d+1}$, for which $R_{\mu \nu}^{\rho \sigma}=$ $-1 / L_{\star}^{2} \cdot\left[\delta_{\mu}^{\rho} \delta_{\nu}^{\sigma}-\delta_{\mu}^{\sigma} \delta_{\nu}^{\rho}\right]$. On such a background - more generally, on any maximally symmetric background - one can show that the variation of any higher-curvature Lagrangian

\footnotetext{
${ }^{15}$ This is not true for all Riemann tensor components. As shown in subsection 3.6, some components do not generate extrinsic curvatures, and a second derivative monomial of the form (3.49) produces only something with the structure $\mathrm{Riem}^{3}+\mathrm{Riem}^{2} K^{2}$, as in (3.58).
} 
with respect to the Riemann tensor is given by

$$
\left.\frac{\partial \mathcal{L}}{\partial R_{\mu \nu \rho \sigma}}\right|_{\mathrm{AdS}}=k_{0}\left[g^{\mu \rho} g^{\sigma \nu}-g^{\mu \sigma} g^{\rho \nu}\right],
$$

where the constant $k_{0}$ is fixed by imposing $\operatorname{AdS}_{d+1}$ to be a solution of the equations of motion of the theory as [63]

$$
k_{0}=-\left.\frac{L_{\star}^{2}}{4 d} \mathcal{L}\right|_{\text {AdS }}
$$

where $\left.\mathcal{L}\right|_{\text {AdS }}$ is the on-shell Lagrangian of the theory evaluated on $\operatorname{AdS}_{d+1}$. Now, it has been argued using different arguments $[9,12,13,68,69]$ that $\left.\mathcal{L}\right|_{\text {AdS }}$ is actually related to the universal coefficient $a^{\star(d)}$ appearing in the EE across spherical regions in general dimensions. For a general CFT in $d$-dimensions, this is given by

$$
\left.S_{\mathrm{EE}}^{(d)}\right|_{\text {sphere }} \supset \begin{cases}(-)^{\frac{d-2}{2}} 4 a^{\star(d)} \log \left(\frac{R}{\delta}\right) & \text { for even } d, \\ (-)^{\frac{d-1}{2}} 2 \pi a^{\star(d)} & \text { for odd } d .\end{cases}
$$

The exact relation for holographic higher-curvature gravities reads

$$
a^{\star(d)}=-\left.\frac{\pi^{d / 2} L_{\star}^{d+1}}{d \Gamma(d / 2)} \mathcal{L}\right|_{\text {AdS }}, \quad \text { so } \quad k_{0}=\frac{\Gamma\left[\frac{d}{2}\right] a^{\star(d)}}{4 \pi^{d / 2} L_{\star}^{d-1}} .
$$

As a consequence, Wald's piece in the HEE formula becomes proportional to the RyuTakayanagi functional in that case, with an overall coefficient controlled by $a^{\star(d)}$. One has

$$
S_{\mathrm{HEE}}=\frac{2 \Gamma\left[\frac{d}{2}\right] a^{\star(d)}}{\pi^{\frac{d-2}{2}} L_{\star}^{d-1}} \int \mathrm{d}^{d-1} y \sqrt{h}+S_{\text {Anomaly }} .
$$

Hence, for theories for which the anomaly piece is absent, all possible universal terms are proportional to the coefficient $a^{\star(d)}$. As we saw above this includes, at the perturbative level, all $\mathcal{L}\left(g_{\mu \nu}, R_{\rho \sigma}\right)$ densities as well as those including a single Riemann tensor. For them, all the different universal coefficients we will consider in this section will modify the Einstein gravity result by the same overall factor given by $a^{\star(d)} / a_{\mathrm{E}}^{\star(d)}$, where $[22,23]$

$$
a_{\mathrm{E}}^{\star(d)}=\frac{\pi^{\frac{d-2}{2}}}{8 \Gamma\left[\frac{d}{2}\right]} \frac{L_{\star}^{d-1}}{G} .
$$

The coefficient $a^{\star(d)}$ can be easily computed for quadratic and cubic theories, yielding

$$
\begin{aligned}
a_{\mathrm{Riem}^{2}}^{\star(d)}= & {\left[1-2 d(d+1) \alpha_{1}-2 d \alpha_{2}-4 \alpha_{3}\right] a_{\mathrm{E}}^{\star(d)}, } \\
a_{\operatorname{Riem}^{3}}^{\star(d)}= & {\left[1+3(d-1) \beta_{1}+12 \beta_{2}+6 d \beta_{3}+6 d(d+1) \beta_{4}+3 d^{2} \beta_{5}+3 d^{2} \beta_{6}\right.} \\
& \left.+3 d^{2}(d+1) \beta_{7}+3 d^{2}(d+1)^{2} \beta_{8}\right] a_{\mathrm{E}}^{\star(d)} .
\end{aligned}
$$

For the reasons explained above, the corrections corresponding to $\alpha_{1}, \alpha_{2}, \beta_{5}, \beta_{6}, \beta_{7}, \beta_{8}$ will appear as overall corrections to the Einstein gravity result with precisely the above 
coefficients for all possible entangling regions. Particularizing to the Gauss-Bonnet and cubic Lovelock cases, one finds

$$
\begin{aligned}
& a_{\mathcal{X}_{4}}^{\star(d)}=\left[1-2(d-2)(d-1) \lambda_{2}\right] a_{\mathrm{E}}^{\star(d)}, \\
& a_{\mathcal{X}_{6}}^{\star(d)}=\left[1+3(d-4)(d-3)(d-2)(d-1) \lambda_{3}\right] a_{\mathrm{E}}^{\star(d)} .
\end{aligned}
$$

In both cases, the corrections are zero below the critical dimensions, as they should, since in those cases the corresponding contributions to the JM functional (4.6) identically vanish. For a general Lovelock theory of the form (4.3), one would have

$$
a_{\text {Lovelock }}^{\star(d)}=\left[1-\sum_{n} n(-1)^{n} \prod_{k=1}^{2(d-1)}(d-k) \lambda_{n}\right] a_{\mathrm{E}}^{\star(d)} .
$$

The result for the charges $a^{\star(d)}$ for Quasi-topological gravity and Einsteinian cubic gravity reads in each case

$$
\begin{aligned}
& a_{\mathrm{QTG}}^{\star(4)}=\left[1+9 \mu_{\mathrm{QTG}}\right] a_{\mathrm{E}}^{\star(4)}, \\
& a_{\mathrm{ECG}}^{\star(3)}=\left[1+3 \mu_{\mathrm{ECG}}\right] a_{\mathrm{E}}^{\star(3)} .
\end{aligned}
$$

\subsection{Spherical regions}

Let us see how the above results for $a^{\star(d)}$ can be obtained from an explicit calculation for spherical entangling surfaces, $\partial A=\mathbb{S}^{d-2}$, using the corresponding HEE functionals. Across spheres, the universal contribution to the entanglement entropy is given, for a general CFT in $d$-dimensions by eq. (5.3).

In the even-dimensional case, the corresponding logarithmic term for a general smooth region is a linear combination of local integrals over the entangling surface weighted by the different trace-anomaly charges $[45,66,70,71]$ - see eq. (5.52) and eq. (5.64) below. One of the integrals involves the Euler density of the entangling surface and the corresponding trace-anomaly coefficient which appears in front is customarily denoted by " $a$ " (or " $A$ " in $d \geq 6$ ). The rest of integrals involve various combinations of the extrinsic curvature of $\partial A$, and therefore all of them vanish for a spherical entangling surface. Hence, the sphere isolates the $a$-type coefficient, and we have simply $a^{\star}=a$ for even $d$.

The nature of $a^{\star}$ is very different in odd dimensions. In that case, it appears as a constant contribution to the EE, and it has an intrinsically non-local nature. In fact, as shown in [72], $a^{\star}$ is proportional to the free energy, $F=-\log Z$, of the corresponding theory evaluated on $\mathbb{S}^{d}$, namely $F_{\mathbb{S}^{d}}=(-)^{\frac{d+1}{2}} 2 \pi a^{\star}$ or, alternatively, to the thermal entropy of the corresponding CFT at a temperature $T=1 /(2 \pi R)$ on the hyperbolic cylinder $\mathbb{R} \times \mathbb{H}^{d-1}[72]$. From an holographic perspective, this means that $a^{\star}$ can be obtained, besides from a direct entanglement entropy calculation, like the one we perform here, either from the Euclidean on-shell action of pure $\operatorname{AdS}_{(d+1)}$ with $\mathbb{S}^{d}$ boundary or from the Wald entropy of $\operatorname{AdS}_{(d+1)}$ with $\mathbb{R} \times \mathbb{H}^{d-1}$ boundary — see also [73, 74].

We write the $\operatorname{AdS}_{d+1}$ metric as

$$
\mathrm{d} s^{2}=\frac{L_{\star}^{2}}{z^{2}}\left[\mathrm{~d} \tau^{2}+\mathrm{d} z^{2}+\mathrm{d} r^{2}+r^{2} \mathrm{~d} \Omega_{d-2}^{2}\right],
$$


where $\mathrm{d} \Omega_{d-2}^{2}$ is the metric of the usual round sphere. Our entangling surface is a sphere $\mathbb{S}^{d-2}$ of radius $r=\ell$ centered at $r=0$. Let us parametrize the RT surface as: $\tau=0$, $z=Z(r)$. Then, unit normals to the surface are given by

$$
n_{1}=\frac{z}{L_{\star}} \partial_{\tau}, \quad n_{2}=\frac{z}{L_{\star} \sqrt{1+Z^{\prime 2}}}\left(Z^{\prime} \partial_{r}-\partial_{z}\right) .
$$

We have already extended these vector fields to a neighborhood of the surface while keeping them normalized. On the surface, one fixes $z=Z(r)$, and $Z^{\prime}(r)$ is well-defined for any $(r, z)$ with $r \in(0, \ell)$. The induced metric on the surface is given by

$$
h_{\mu \nu} \mathrm{d} x^{\mu} \mathrm{d} x^{\nu}=\frac{L_{\star}^{2}}{Z^{2}}\left[\frac{1}{1+Z^{\prime 2}}\left(\mathrm{~d} r+Z^{\prime} \mathrm{d} z\right)^{2}+r^{2} \mathrm{~d} \Omega_{d-2}^{2}\right] .
$$

With these results one can compute in full generality the components of the extrinsic curvatures,

$$
\begin{aligned}
K^{1}{ }_{\mu \nu} & =0, \\
K_{r r}^{2} & =\frac{L_{\star}}{Z^{2}\left(1+Z^{\prime 2}\right)^{5 / 2}}\left(1+Z^{\prime 2}+Z Z^{\prime \prime}\right), \quad K_{r z}^{2}=Z^{\prime} K_{r r}^{2}, \\
K^{2}{ }_{z z} & =Z^{\prime 2} K_{r r}^{2}, \quad K^{2}{ }_{m n}=\frac{L_{\star}}{Z^{2} \sqrt{1+Z^{\prime 2}}}\left(Z Z^{\prime}+r\right) r \hat{g}_{m n},
\end{aligned}
$$

where $\hat{g}_{m n}$ is the metric of the unit $\mathbb{S}^{d-2}$. Obtaining the traces is now easy. $K^{1}=0$ trivially, whereas

$$
K^{2}=\frac{1}{L_{\star} r\left(1+Z^{\prime 2}\right)^{3 / 2}}\left[r Z Z^{\prime \prime}+(d-2) Z Z^{\prime}\left(1+Z^{\prime 2}\right)+(d-1) r\left(1+Z^{\prime 2}\right)\right] .
$$

The vanishing of this trace is exactly the differential equation for the surface one would obtain by minimizing the RT functional, which in this case reads

$$
S_{\mathrm{HEE}}^{\mathrm{E}}=\frac{L_{\star}^{d-1} \pi^{(d-1) / 2}}{2 G \Gamma\left[\frac{d-1}{2}\right]} \int_{0}^{\ell} \mathrm{d} r \frac{r^{d-2}}{Z^{d-1}} \sqrt{1+Z^{\prime 2}} .
$$

The solution for this differential equation satisfying the boundary condition $Z(\ell)=0$ is $r^{2}+Z^{2}=\ell^{2}$. The simplicity of this RT surface has another important consequence: since $Z Z^{\prime}=-r$ and $Z Z^{\prime \prime}=-\left(1+Z^{\prime 2}\right)$, the extrinsic curvature $K^{2}{ }_{\mu \nu}$ vanishes. Thus, both $K_{\mu \nu}^{1}$ and $K^{2}{ }_{\mu \nu}$ are zero for the RT surface. Now, since the anomaly term in the general highercurvature functional is quadratic in extrinsic curvatures of the surface, when minimizing the functional, the RT surface will also be extremal for the full functional if we were to consider it fully non-perturbatively. ${ }^{16}$

In order to compute the universal contribution to the HEE the last step is to regulate eq. (5.20), e.g., by writing

$$
S_{\mathrm{HEE}}^{\mathrm{E}}=\frac{L_{\star}^{d-1} \pi^{(d-1) / 2}}{2 G \Gamma\left[\frac{d-1}{2}\right]} \int_{\delta / \ell}^{1} \mathrm{~d} y \frac{\left(1-y^{2}\right)^{(d-3) / 2}}{y^{d-1}},
$$

\footnotetext{
${ }^{16}$ This is true irrespective of the splitting used.
} 
where we introduced a cutoff at $z=\delta$. Integrating by parts, it is easy to show that for odd $d$ we get a constant term while for even $d$ we get a logarithmic one. The final result takes the form eq. (5.3), plus a series of non-universal divergent pieces of the form $(\ell / \delta)^{(d-2 k)}$ with $k=1,2, \ldots,(d-1) / 2$ for odd $d$ and $k=1,2, \ldots,(d-2) / 2$ for even $d$ - see e.g., [22] for the numerical coefficients. When higher-curvature terms are included, the vanishing of $K^{1}{ }_{\mu \nu}$ and $K^{2}{ }_{\mu \nu}$ makes the result reduce to the corresponding Wald piece, which in turn reduces to an overall constant proportional to $\left.\mathcal{L}\right|_{\text {AdS }}$ via eq. (5.2) times the Einstein gravity result. Hence, we are left again with eq. (5.3) where $a^{\star}$ is given by eq. (5.4) in each case.

\subsection{Slab regions}

Let us consider now an entangling region consisting of a slab of width $\ell$ along a particular dimension, $x \in[\ell / 2, \ell / 2]$, and infinite along the remaining $(d-2)$. For general theories, the EE in that case takes the form

$$
S_{\mathrm{EE}}=\xi \frac{L_{y}^{d-2}}{\delta^{d-2}}-\kappa^{(d)} \frac{L_{y}^{d-2}}{\ell^{d-2}},
$$

where $\xi$ is a non-universal constant. As opposed to other universal EE contributions considered here, $\kappa^{(d)}$ does not have any (known) alternative interpretation beyond EE. For instance, it is not expected to be related to charges characterizing simple local correlators. Previous papers where $\kappa^{(d)}$ was computed for certain holographic higher-curvature gravities include [65], where it was evaluated for quadratic theories in $d=3$, and [64], where it was computed for Gauss-Bonnet gravity in $d=4$ fully nonperturbatively using the JM functional.

We write the $\mathrm{AdS}_{d+1}$ metric as

$$
\mathrm{d} s^{2}=\frac{L_{\star}^{2}}{z^{2}}\left[\mathrm{~d} \tau^{2}+\mathrm{d} z^{2}+\mathrm{d} x^{2}+\mathrm{d} \vec{y}_{d-2}^{2}\right] .
$$

The RT surface will be invariant under translations along the $(d-2)$ transverse directions, so we can parametrize it by $z=Z(x)$. Unit normals to the surface will be given by

$$
n_{1}=\frac{z}{L_{\star}} \partial_{\tau}, \quad n_{2}=\frac{z}{L_{\star} \sqrt{1+Z^{\prime 2}}}\left(Z^{\prime} \partial_{x}-\partial_{z}\right) .
$$

The induced metric on the surface is now given by

$$
h_{\mu \nu} \mathrm{d} x^{\mu} \mathrm{d} x^{\nu}=\frac{L_{\star}^{2}}{Z^{2}}\left[\frac{1}{1+Z^{\prime 2}}\left(\mathrm{~d} x+Z^{\prime} \mathrm{d} z\right)^{2}+\mathrm{d} \vec{y}_{d-2}^{2}\right] .
$$

The non-vanishing components of the extrinsic curvatures $K_{\mu \nu}^{a}$ read

$$
K_{x x}^{2}=\frac{L_{\star}\left(1+Z^{\prime 2}+Z Z^{\prime \prime}\right)}{Z^{2}\left(1+Z^{\prime 2}\right)^{5 / 2}}=\frac{K_{x z}^{2}}{Z^{\prime}}=\frac{K^{2} z z}{Z^{\prime 2}}, \quad K_{m n}^{2}=\frac{L_{\star} \delta_{m n}}{Z^{2} \sqrt{1+Z^{\prime 2}}},
$$

whereas all components of $K_{\mu \nu}^{1}$ vanish.

Projectors on the surface are given by

$$
t_{x}=Z^{\prime} \partial_{z}+\partial_{x}, \quad t_{m}=\partial_{m}, \quad \forall m=1, \ldots, d-2 .
$$


Using these, we find

$$
h_{i j} \mathrm{~d} y^{i} \mathrm{~d} y^{j}=\frac{L_{\star}^{2}}{Z^{2}}\left[\left(1+Z^{\prime 2}\right) \mathrm{d} x^{2}+\mathrm{d} \vec{y}_{d-2}^{2}\right] .
$$

Also, the non-vanishing components of $K_{i j}^{2}$ read (note the slight abuse of notation)

$$
K_{x x}^{2}=\frac{L_{\star}\left(1+Z^{\prime 2}+Z Z^{\prime \prime}\right)}{Z^{2} \sqrt{1+Z^{\prime 2}}}, \quad K_{m n}^{2}=\frac{L_{\star}}{Z^{2} \sqrt{1+Z^{\prime 2}}} \delta_{m n} .
$$

With these building blocks we can compute all the different pieces appearing in the corresponding EE functionals. For instance, the relevant expressions for the quadratic ones read

$$
\begin{aligned}
K_{i j}^{a} K^{a i j} & =\frac{(d-1)\left(1+Z^{\prime 2}\right)^{2}+Z^{2} Z^{\prime 2}+2\left(1+Z^{\prime 2}\right) Z Z^{\prime \prime}}{L_{\star}^{2}\left(1+Z^{\prime 2}\right)^{3}}, \\
R_{a b}^{a b} & =-\frac{2}{L_{\star}^{2}}, \quad R=-\frac{d(d+1)}{L_{\star}^{2}}, \quad R_{a}^{a}=-\frac{2 d}{L_{\star}^{2}} .
\end{aligned}
$$

Now, the Ryu-Takayanagi surface is determined by the condition $K^{2}=0$, where in this case we have

$$
K^{2}=\frac{(d-1)\left(1+Z^{\prime 2}\right)+Z Z^{\prime \prime}}{L_{\star}\left(1+Z^{\prime 2}\right)^{3 / 2}} .
$$

A first integral can be shown to exist so that

$$
Z^{\prime}=-\frac{\sqrt{z_{\star}^{2(d-1)}-Z^{2(d-1)}}}{Z^{(d-1)}}, \quad \text { where } \quad z_{\star}=\frac{\Gamma\left[\frac{1}{2(d-1)}\right]}{2 \sqrt{\pi} \Gamma\left[\frac{d}{2(d-1)}\right]} \ell
$$

is the value of $z$ corresponding to the turning point of the surface. Now, after some massaging, the EE for Einstein gravity can be seen to be given by [22, 23]

$$
S_{\mathrm{HEE}}^{\mathrm{E}}=\frac{L_{\star}^{d-1} L_{y}^{d-2}}{2 G z_{\star}^{d-2}} \int_{\delta}^{1} \frac{\mathrm{d} y}{y^{d-1} \sqrt{1-y^{2(d-1)}}}=\xi_{\mathrm{E}} \frac{L_{y}^{d-2}}{\delta^{d-2}}-\kappa_{\mathrm{E}}^{(d)} \frac{L_{y}^{d-2}}{\ell^{d-2}},
$$

where $L_{y}$ are IR regulators for the $(d-2)$ transverse directions. The universal and nonuniversal constants $\kappa_{\mathrm{E}}^{(d)}$ and $\xi_{\mathrm{E}}$ read respectively

$$
\kappa_{\mathrm{E}}^{(d)}=\frac{2^{d-3} \pi^{\frac{d-1}{2}} \Gamma\left[\frac{d}{2(d-1)}\right]^{d-1}}{(d-2) \Gamma\left[\frac{1}{2(d-1)}\right]^{d-1}} \frac{L_{\star}^{d-1}}{G}, \quad \xi_{\mathrm{E}}=\frac{L_{\star}^{d-1}}{2(d-2) G} .
$$

Let us see how these generalize when quadratic and cubic terms are introduced. For a general quadratic theory of the form (4.8) one finds

$$
S_{\mathrm{HEE}}^{\mathrm{Riem}^{2}}=\frac{L_{\star}^{d-1} L_{y}^{d-2}}{2 G z_{\star}^{d-2}} \int_{\delta}^{1} \frac{\left[1-2 d(d+1) \alpha_{1}-2 d \alpha_{2}-2 \alpha_{3}\left[2+(d-1)(d-2) y^{2(d-1)}\right]\right]}{y^{d-1} \sqrt{1-y^{2(d-1)}}} \mathrm{d} y,
$$

and from this,

$$
S_{\mathrm{HEE}}^{\mathrm{Riem}}{ }^{2}=\xi_{\mathrm{Riem}^{2}} \frac{L_{y}^{d-2}}{\delta^{d-2}}-\kappa_{\mathrm{Riem}^{2}}^{(d)} \frac{L_{y}^{d-2}}{\ell^{d-2}}
$$


where now $\xi_{\text {Riem }}^{2}$ gets a factor identical to the one of $a_{\mathrm{Riem}^{2}}^{\star(d)}$ whereas the universal coefficient reads

$$
\kappa_{\mathrm{Riem}^{2}}^{(d)}=\left[1-2 d(d+1) \alpha_{1}-2 d \alpha_{2}+2(d-3)[2+d(d-2)] \alpha_{3}\right] \kappa_{\mathrm{E}}^{(d)} .
$$

Note that there are two kinds of terms in the integrand. On the one hand, pieces arising from purely intrinsic curvatures are proportional to the Einstein gravity one, which is of the form $\sim 1 /\left(y^{d-1} \sqrt{1-y^{2(d-1)}}\right)$. On the other hand, the contribution which involves two extrinsic curvatures has an extra $\sim y^{2(d-1)}$ factor. It is easy to see that $\xi_{\mathrm{Riem}^{2}}$ is unaffected by the second type of terms, which explains why the same prefactor as for $a_{\mathrm{Riem}^{2}}^{*}$ appears in that case. Nevertheless, recall that $\xi$ is not a universal quantity (we can modify it by changing the regulator), so its interest is very limited. On the other hand, the universal constant $\kappa_{\mathrm{Riem}^{2}}^{(d)}$ does get affected by the extrinsic curvature term. The result for $\kappa_{\mathrm{Riem}^{2}}^{(3)}$ agrees with the one obtained in [65], as it should.

We find a similar kind of behavior for the cubic theories. Wald-like terms produce contributions proportional to the Einstein gravity result, and the non-universal constant $\xi_{\mathrm{Riem}^{3}}$ is proportional to $a_{\mathrm{Riem}^{3}}^{\star(d)}$, namely, $\xi_{\mathrm{Riem}^{3}} / \xi_{\mathrm{E}}=a_{\mathrm{Riem}^{3}}^{\star(d)} / a_{\mathrm{E}}^{\star(d)}$. On the other hand, terms with two extrinsic curvatures have an extra factor $\sim y^{2(d-1)}$ in the integrand, and those with four, one of the form $\sim y^{4(d-1)}$. Both types of terms affect the universal coefficient. ${ }^{17}$ The final result reads

$$
\begin{aligned}
\kappa_{\mathrm{Riem}^{3}}^{(d)}= & {\left[1+3\left[d-1+(d-1)(d-2)^{2}+\frac{d(d-2)^{2}\left(8+d\left(d^{2}-9\right)\right)}{8(2 d-1)}\right] \beta_{1}\right.} \\
& +12\left[1-(d-1)(d-2)^{2}+\frac{d(d-1)(d-2)^{2}(7+d(d-5))}{4(2 d-1)}\right] \beta_{2} \\
& +2 d\left[3-(d-1)(d-2)^{2}\right] \beta_{3}+2 d(d+1)\left[3-(d-1)(d-2)^{2}\right] \beta_{4} \\
& \left.+3 d^{2} \beta_{5}+3 d^{2} \beta_{6}+3 d^{2}(d+1) \beta_{7}+3 d^{2}(d+1)^{2} \beta_{8}\right] \kappa_{\mathrm{E}}^{(d)} .
\end{aligned}
$$

A check of these results for $\kappa_{\mathrm{Riem}^{2}}^{(d)}$ and $\kappa_{\mathrm{Riem}^{3}}^{(d)}$ can be performed by particularizing them to Lovelock theories, for which the JM formula in eq. (4.6) can be alternatively used. We find

$$
\begin{aligned}
\kappa_{\mathcal{X}_{4}}^{(d)} & =\left[1+2(d-3)(d-2)(d-1) \lambda_{2}\right] \kappa_{\mathrm{E}}^{(d)}, \\
\kappa_{\mathcal{X}_{6}}^{(d)} & =\left[1-\frac{3(d-5)(d-4)(d-3)(d-2)(d-1)^{2}}{(2 d-1)} \lambda_{3}\right] \kappa_{\mathrm{E}}^{(d)},
\end{aligned}
$$

which precisely agree with the ones obtained using eq. (4.6). Observe that the corrections to the Einstein gravity result vanish in dimensions lower or equal to the critical one, i.e., for $d+1 \leq 2 n$. One can also verify that $\kappa_{\mathcal{X}_{4}}^{(4)}$ agrees with the nonperturbative result found

\footnotetext{
${ }^{17}$ Effectively every extra factor $y^{2(d-1)}$ can be replaced by a $(2-d)$ factor as far as $\kappa^{(d)}$ is concerned, and every extra $y^{4(d-1)}$ can be replaced by a $d(2-d) /(2 d-1)$. If we repeated the calculation including general order- $n$ higher-curvature pieces, we would obtain extra factors generally involving all even powers of $y$ up to $y^{2(n-1)(d-1)}$.
} 
in [64] at leading order in $\lambda_{2}$. For Quasi-topological and Einsteinian cubic gravity we find, respectively

$$
\begin{aligned}
\kappa_{\mathrm{QTG}}^{(4)} & =\left[1+9 \mu_{\mathrm{QTG}}\right] \kappa_{\mathrm{E}}^{(4)}, \\
\kappa_{\mathrm{ECG}}^{(3)} & =\left[1+3 \mu_{\mathrm{ECG}}\right] \kappa_{\mathrm{E}}^{(3)} .
\end{aligned}
$$

As mentioned above, the coefficient $\kappa^{(d)}$ does not have an alternative interpretation beyond entanglement entropy, which is manifest in this case from the fact that in all cases in which various coefficients characterizing the dual theory have been computed for some of the above theories, all the corresponding values differ from the ones obtained here for $\kappa^{(d)}{ }^{18}$ This includes, in particular, all the rest of coefficients computed in this paper $(c, a$ in $d=4 ; A, B_{1}, B_{2}, B_{3}$ in $d=6$; $a^{\star(d)}$ in general $d$; the corner charge $\sigma$ in $d=3$ ) as well as others like the stress-tensor two-point function charge $C_{T}$, the coefficient $C_{S}$ relating the thermal entropy of a plasma to its temperature, as well as others arising in the context of holographic complexity [7, 8, 28, 36, 45, 64-67].

\subsection{Cylinder regions}

Let us now consider (hyper)cylindrical entangling surfaces. We will be mostly interested in the universal logarithmic piece arising for such regions in $d=4$ and $d=6$ theories. We write the Euclidean $\operatorname{AdS}_{(d+1)}$ metric as

$$
\mathrm{d} s^{2}=\frac{L_{\star}^{2}}{z^{2}}\left[\mathrm{~d} \tau^{2}+\mathrm{d} z^{2}+\mathrm{d} \vec{y}_{(d-3-j)}^{2}+\mathrm{d} r^{2}+r^{2} \mathrm{~d} \Omega_{(j+1)}^{2}\right],
$$

where $\mathrm{d} \Omega_{(j+1)}^{2}$ is the metric of a round $(j+1)$-dimensional sphere. Our entangling regions will be parametrized by $\tau=0, r=R_{0}$, with $j$ taking values $j=0, \ldots, d-3$, which correspond to entangling surfaces $\partial A=\mathbb{S}^{1} \times \mathbb{R}^{d-3}, \mathbb{S}^{2} \times \mathbb{R}^{d-4}, \ldots, \mathbb{S}^{d-3} \times \mathbb{R}^{1}, \mathbb{S}^{d-2}$, respectively.

We parametrize the RT surface as $r=R(z)$. Unit normals and projectors on the surface read

$$
n_{1}=\frac{z}{L_{\star}} \partial_{\tau}, \quad n_{2}=\frac{z}{L_{\star} \sqrt{1+R^{\prime 2}}}\left(R^{\prime} \partial_{z}-\partial_{r}\right), \quad t_{z}=\partial_{z}+R^{\prime} \partial_{r}, t_{m}=\partial_{m}, t_{\phi}=\partial_{\phi},
$$

where $m=1, \ldots, d-3-j$ and $\phi=1, \ldots, j+1$. The induced metric reads

$$
h_{i j} \mathrm{~d} y^{i} \mathrm{~d} y^{j}=\frac{L_{\star}^{2}}{z^{2}}\left[\left(1+R^{\prime 2}\right) \mathrm{d} z^{2}+\mathrm{d} \vec{y}_{(d-3-j)}^{2}+R^{2} d \Omega_{(j+1)}^{2}\right] .
$$

The non-vanishing components of $K_{i j}^{2}$ read now

$$
\begin{aligned}
K_{z z}^{2} & =\frac{-L_{\star}\left(R^{\prime}+R^{\prime 3}-z R^{\prime \prime}\right)}{z^{2} \sqrt{1+R^{\prime 2}}}, \quad K_{m n}^{2}=\frac{-L_{\star} R^{\prime} \delta_{m n}}{z^{2} \sqrt{1+R^{\prime 2}}}, \\
K_{\phi_{j} \phi_{k}}^{2} & =\frac{-L_{\star}\left(z+R R^{\prime}\right) R}{z^{2} \sqrt{1+R^{\prime 2}}} \prod_{l=1}^{j-1} \sin ^{2} \phi_{l} \delta_{j k} .
\end{aligned}
$$

\footnotetext{
${ }^{18}$ The exception is the sharp-limit corner coefficient $\kappa$, which can be shown to coincide with $\kappa^{(3)}$ on general grounds, as explained below.
} 
The equation for the RT surface is, as usual, $K^{2}=0$, where

$$
K^{2}=\frac{\left(R R^{\prime \prime}-(j+1)\right) z-(d-1) R R^{\prime}\left(1+R^{\prime 2}\right)-(j+1) z R^{\prime 2}}{L_{\star} R\left(1+R^{\prime 2}\right)^{3 / 2}} .
$$

In the case of Einstein gravity, the RT functional reduces to

$$
S_{\mathrm{EE}}^{\mathrm{E}}=\frac{L_{\star}^{d-1} L_{y}^{d-3-j} \Omega_{(j+1)}}{4 G} \int_{\delta}^{z_{\max }} \mathrm{d} z \frac{R^{j+1}}{z^{d-1}} \sqrt{1+R^{\prime 2}},
$$

where $\Omega_{(j+1)} \equiv 2 \pi^{(j+2) / 2} / \Gamma[(j+2) / 2]$. As anticipated, we are interested in the logarithmic contribution to the entanglement entropy in even dimensional theories. Such a contribution is local in the entangling surface $\partial A$ so, from the holographic perspective, it suffices to consider a perturbative solution to $K^{2}=0$ near the boundary. The result reads ${ }^{19}$

$$
R(z)=R_{0}-\frac{(j+1)}{2 R_{0}(d-2)} z^{2}+\mathcal{O}\left(z^{4}\right)
$$

which we need to plug back into our functionals.

\subsubsection{Four dimensions}

For general CFTs in four dimensions, the universal contribution to the entanglement entropy for a smooth entangling surface characterized by some scale $\ell$ is given by Solodukhin's formula $[70,71]$

$$
S_{\mathrm{EE}}^{(4)} \supset-\frac{1}{2 \pi} \int_{\partial A} \mathrm{~d}^{2} y \sqrt{\gamma}\left[a \mathcal{R}+c\left(\operatorname{tr} k^{2}-\frac{1}{2} k^{2}\right)\right] \log \left(\frac{\ell}{\delta}\right),
$$

where $\mathcal{R}$ is the Ricci scalar of the induced metric induced on $\partial A, \gamma_{i j}$, and here and in the next subsection we use the notation $k \equiv \gamma^{i j} k_{i j}$ and $\operatorname{tr} k^{n} \equiv k_{i_{1}}^{i_{2}} k_{i_{2}}^{i_{3}} \ldots k_{i_{n}}^{i_{1}}$, where $k_{i j}$ is the extrinsic curvature. $a$ and $c$ are the coefficients appearing in the usual trace-anomaly expression [75]

$$
\left\langle T_{a}^{a}\right\rangle=-\frac{a}{16 \pi^{2}} \mathcal{X}_{4}+\frac{c}{16 \pi^{2}} C_{a b c d} C^{a b c d},
$$

where $\mathcal{X}_{4}$ and $C_{a b c d}$ are the Euler density and Weyl tensor of the curved manifold in which the CFT is considered.

Let us then start considering our holographic functionals for $d=4$ and $j=0$. For eq. (5.50) one finds

$$
S_{\mathrm{HEE}}^{\mathrm{E}}=\frac{\pi L_{\star}^{3} L_{y}}{2 G} \int_{\delta}^{z_{\max }} \mathrm{d} z\left[\frac{R_{0}}{z^{3}}-\frac{1}{8 R_{0} z}+\ldots\right]=\cdots-\frac{c_{\mathrm{E}}}{2} \frac{L_{y}}{R_{0}} \log \left(R_{0} / \delta\right)+\ldots
$$

where

$$
c_{\mathrm{E}}=\frac{\pi L_{\star}^{3}}{8 G} .
$$

\footnotetext{
${ }^{19}$ When performing this expansion, it does not seem to be possible to solve the equation beyond quadratic order for $d=4$ and beyond quartic order in $d=6$. While this does not affect our calculations, it would be interesting to better understand the origin of this issue.
} 
This takes the form expected for a cylinder region in general CFTs, where the value of $c_{\mathrm{E}}$ matches the corresponding trace anomaly charge. In our conventions, this is in turn related to the stress-tensor two-point function charge ${ }^{20} C_{T}$ through $c=\pi^{4} C_{T} / 40$ for general theories - compare with $C_{T}^{\mathrm{E}}$ in eq. (5.59).

Performing the analogous calculations for quadratic and cubic theories, we observe that introducing the expansion eq. (5.51) in the corresponding functionals there are three kinds of terms which appear multiplying the Einstein gravity integrand in eq. (5.54): terms coming from the Wald pieces, which are constant; terms involving products of two extrinsic curvatures, which are $\sim z^{2}$; and terms involving products of four extrinsic curvatures, which go with $\sim z^{4}$. Terms of the latter kind do not contribute to $c$, which is a manifestation of the splitting-independent nature of this coefficient. The final result for $c_{\mathrm{Riem}^{2}}$ and $c_{\mathrm{Riem}}{ }^{3}$ reads

$$
\begin{aligned}
& c_{\text {Riem }^{2}}=\left[1-40 \alpha_{1}-8 \alpha_{2}+4 \alpha_{3}\right] c_{\mathrm{E}}, \\
& c_{\text {Riem }^{3}}=\left[1+21 \beta_{1}-36 \beta_{2}-8 \beta_{3}-40 \beta_{4}+48 \beta_{5}+48 \beta_{6}+240 \beta_{7}+1200 \beta_{8}\right] c_{\mathrm{E}} .
\end{aligned}
$$

These are again in agreement with the general relation with $C_{T}$. Indeed, for general quadratic and cubic theories in $d$-dimensions one finds

$$
\begin{aligned}
C_{T}^{\mathrm{Riem}^{2}}= & {\left[1-2 d(d+1) \alpha_{1}-2 d \alpha_{2}+4(d-3) \alpha_{3}\right] C_{T}^{\mathrm{E}} } \\
C_{T}^{\mathrm{Riem}^{3}}= & {\left[1+3(3 d-5) \beta_{1}-12(2 d-5) \beta_{2}-2 d(2 d-7) \beta_{3}-2 d(2 d-7)(d+1) \beta_{4}+3 d^{2} \beta_{5}\right.} \\
& \left.+3 d^{2} \beta_{6}+3 d^{2}(d+1) \beta_{7}+3 d^{2}(d+1)^{2} \beta_{8}\right] C_{T}^{\mathrm{E}},
\end{aligned}
$$

where the Einstein gravity result reads

$$
C_{T}^{\mathrm{E}}=\frac{\Gamma[d+2]}{8(d-1) \Gamma\left[\frac{d}{2}\right] \pi^{\frac{d+2}{2}}} \frac{L_{\star}^{d-1}}{G} .
$$

These results for $C_{T}$ can be obtained in different ways. A simple one consists in computing the linearized equations of the theory around an AdS background. For a general higher-curvature gravity, these are fourth-order equations which describe the dynamics of a massive scalar mode and a ghost-like massive graviton in addition to the usual general relativity massless graviton. The resulting equations can be characterized in terms of the masses of the new two modes as well as an effective Newton constant [63, 77]. This generically takes the form $G_{\text {eff }}=G / \gamma$, where $\gamma$ depends on the higher-curvature couplings. Via holography, a rescaling of $G$ is equivalent to a rescaling of the stress-tensor charge $C_{T}$, which becomes $\gamma C_{T}^{\mathrm{E}}$. $G_{\text {eff }}$ was computed in [63] explicitly for general quadratic, cubic and quartic gravities in general dimensions, so we can easily obtain the values of $C_{T}$ shown above. In the particular cases of Lovelock, Quasi-topological and Einsteinian cubic gravity

\footnotetext{
${ }^{20}$ This is defined as the only theory-dependent content of the stress-tensor correlator, which otherwise is completely fixed by conformal symmetry [76]. For a general CFT in $d$-dimensions one finds $\left\langle T_{a b}(x) T_{c d}(0)\right\rangle=$ $C_{T} I_{a b, c d}(x) / x^{2 d}$, where $I_{a b, c d}(x)$ is a fixed tensorial structure.
} 
densities, they reduce to

$$
\begin{aligned}
C_{T}^{\mathcal{X}_{4}} & =\left[1-2(d-2)(d-3) \lambda_{2}\right] C_{T}^{\mathrm{E}} \\
C_{T}^{\mathcal{X}_{6}} & =[1+3(d-2)(d-3)(d-4)(d-5)] C_{T}^{\mathrm{E}}, \\
C_{T}^{\mathrm{QTG}} & =\left[1-3 \mu_{\mathrm{QTG}}\right] C_{T}^{\mathrm{E}}, \\
C_{T}^{\mathrm{ECG}} & =\left[1-3 \mu_{\mathrm{ECG}}\right] C_{T}^{\mathrm{E}} .
\end{aligned}
$$

Note that all these differ from the slab coefficients $\kappa^{(d)}$ computed in the previous subsection.

\subsubsection{Six dimensions}

Let us now turn to six dimensions. In this case, a similar expression for the logarithmic term involving the trace anomaly coefficients holds for general CFTs, and is given by $[45,66]$

$$
\begin{array}{r}
S_{\mathrm{EE}}^{(6)} \supset \int_{\partial A} \mathrm{~d}^{4} y \sqrt{\gamma}\left[2 A \mathcal{X}_{4}+\frac{3 \pi}{2} B_{1}\left(3 T_{1}-2 T_{2}\right)-12 \pi B_{2} T_{2}\right. \\
\left.+6 \pi B_{3}\left(T_{3}+9 T_{1}-12 T_{2}\right)\right] \log \left(\frac{\ell}{\delta}\right)
\end{array}
$$

where $\mathcal{X}_{4}$ is the Euler density associated to the induced metric $\gamma_{i j}$ and now

$$
\begin{aligned}
& T_{1} \equiv\left(\operatorname{tr} k^{2}\right)^{2}-\frac{1}{2} k^{2} \operatorname{tr} k^{2}+\frac{1}{16} k^{4}, \\
& T_{2} \equiv \operatorname{tr} k^{4}-k \operatorname{tr} k^{3}+\frac{3}{8} k^{2} \operatorname{tr} k^{2}-\frac{3}{64} k^{4}, \\
& T_{3} \equiv\left(\nabla_{i} k\right)^{2}-\frac{25}{16} k^{4}+11 k^{2} \operatorname{tr} k^{2}-6\left(\operatorname{tr} k^{2}\right)^{2}-16 k \operatorname{tr} k^{3}+12 \operatorname{tr} k^{4} .
\end{aligned}
$$

Similarly, the coefficients $A, B_{1}, B_{2}$ and $B_{3}$ are the ones appearing in the trace anomaly, which in this case takes the form [78-81]

$$
\left\langle T_{a}^{a}\right\rangle=\sum_{i=1}^{3} B_{i} I_{i}+2 A \mathcal{X}_{6}
$$

where $\mathcal{X}_{6}$ is the Euler density and the $I_{i}$ are cubic conformal invariants given by

$$
\begin{aligned}
I_{1} & \equiv C_{d a b c} C^{a e f b} C_{e}{ }^{d c} f, \quad I_{2} \equiv C_{a b}{ }^{c d} C_{c d}{ }^{e f} C_{e f}{ }^{a b}, \\
I_{3} & \equiv C_{a c e g}\left(\nabla^{2} \delta_{b}^{a}+4 R_{b}^{a}-\frac{6}{5} R \delta_{b}^{a}\right) C^{b c e g} .
\end{aligned}
$$

For the entangling regions we are considering here, the induced metric on $d=6$ Minkowski space reads

$$
d s_{\gamma}^{2}=\mathrm{d} \vec{y}_{(3-j)}^{2}+R_{0}^{2} \mathrm{~d} \Omega_{(j+1)}^{2} .
$$

The relevant expressions for the extrinsic curvature invariants read

$$
k=\frac{(j+1)}{R_{0}}, \quad \operatorname{tr} k^{n}=\frac{(j+1)}{R_{0}^{n}},
$$


and from this, one finds

$$
\begin{aligned}
& \mathcal{X}_{4}=\frac{(j-2)(j-1) j(j+1)}{R_{0}^{4}}, \\
& T_{1}=\frac{(j-3)^{2}(j+1)^{2}}{16 R_{0}^{4}}, \\
& T_{2}=-\frac{(j-3)(j+1)(7+3 j(j-2))}{64 R_{0}^{4}}, \\
& T_{3}=\frac{(j-3)(j+1)(3+j(26-25 j)}{16 R_{0}^{4}},
\end{aligned}
$$

where, for completeness, we also included the value of $\mathcal{X}_{4}$ which vanishes for all the cylinderlike regions $(j=0,1,2)$. Then, the entanglement entropy universal term reduces, for general CFTs, to

$$
\begin{aligned}
S_{\mathrm{EE}} \supset \frac{(j+1) \Omega_{(j+1)}}{64}[ & {\left[128 A j(j-1)(j-2)+3 \pi(j-3)\left[B_{1}(9 j(j-2)-11)\right.\right.} \\
& \left.\left.+4 B_{2}(3 j(j-2)+7)-8 B_{3}(j+1)(3+7 j)\right]\right] \frac{L_{y}^{3-j}}{R_{0}^{3-j}} \log \left(\frac{\ell}{\delta}\right) .
\end{aligned}
$$

On the other hand, the holographic result for Einstein gravity reads

$$
\begin{aligned}
S_{\mathrm{HEE}}^{\mathrm{E}} & =\frac{L_{\star}^{5} L_{y}^{3-j} \Omega_{(j+1)}}{4 G} \int_{\delta}^{z_{\max }} \mathrm{d} z\left[\frac{R_{0}^{j+1}}{z^{5}}-\frac{3(j+1)^{2} R_{0}^{j-1}}{32 z^{3}}+\frac{(j+1)^{3}(7 j-9)}{2048 R_{0}^{3-j} z}+\ldots\right], \\
& =\cdots+\frac{(1+j)^{3}(7 j-9) \Omega_{(j+1)} L_{\star}^{5}}{8192 G} \frac{L_{y}^{3-j}}{R_{0}^{3-j}} \log \left(\frac{\ell}{\delta}\right)+\cdots
\end{aligned}
$$

Comparing with eq. (5.77) for $j=0,1,2,3$ we can obtain the Einstein gravity values of $A$, $B_{1}, B_{2}, B_{3}$. The results read

$$
A_{\mathrm{E}}=\frac{L_{\star}^{5}}{512 G}, \quad B_{1}^{\mathrm{E}}=-\frac{L_{\star}^{5}}{256 \pi G}, \quad B_{2}^{\mathrm{E}}=-\frac{L_{\star}^{5}}{1024 \pi G}, \quad B_{3}^{\mathrm{E}}=\frac{L_{\star}^{5}}{3072 \pi G},
$$

in agreement with previous calculations $[66,82]$. In particular, the value of the $A$ charge satisfies $A_{\mathrm{E}}=a_{\mathrm{E}}^{\star(6)} /\left(32 \pi^{2}\right)$, a relation which holds for general theories in the present conventions. In particular, the values of $A$ for all the rest of holographic higher-curvature theories are proportional to the corresponding coefficients $a^{\star(6)}$.

Moving to quadratic theories, the contributions without anomaly piece modify the charges in the same way as $a^{\star(6)}$, whereas the term involving two Riemanns contains an extra piece coming from a contraction of extrinsic curvatures, which in this case reads

$$
K_{a i j} K^{a i j}=-\frac{(j-3)(j+1)}{4 L_{\star}^{2} R_{0}^{2}} z^{2}+\frac{(j-3)^{2}(j+1)^{2}}{64 L_{\star}^{2} R_{0}^{4}} z^{4}+\ldots
$$


Putting the pieces together in the quadratic functional eq. (4.10) and again comparing with eq. (5.77) we find

$$
\begin{aligned}
& B_{1}^{\mathrm{Riem}^{2}}=\left[1-84 \alpha_{1}-12 \alpha_{2}+\frac{4}{3} \alpha_{3}\right] B_{1}^{\mathrm{E}}, \\
& B_{2}^{\mathrm{Riem}^{2}}=\left[1-84 \alpha_{1}-12 \alpha_{2}-\frac{28}{3} \alpha_{3}\right] B_{2}^{\mathrm{E}}, \\
& B_{3}^{\mathrm{Riem}^{2}}=\left[1-84 \alpha_{1}-12 \alpha_{2}+12 \alpha_{3}\right] B_{3}^{\mathrm{E}} .
\end{aligned}
$$

We have verified that these results reduce to the ones found in [83] for seven-dimensional Critical Gravity [84, 85]. In that case, $\alpha_{1}=-1 / 240, \alpha_{2}=1 / 20, \alpha_{3}=-1 / 16$ and the charges read $B_{1}^{\mathrm{CG}}=2 / 3, B_{1}^{\mathrm{CG}}=4 / 3, B_{3}^{\mathrm{CG}}=0$. It is also easy to verify that the resulting charges satisfy the relation $3 B_{3}=\left(B_{2}-B_{1} / 2\right)$, which holds for theories that are unaffected by the splittings choice, as argued in [45].

Proceeding analogously with the cubic densities, we obtain

$$
\begin{aligned}
& B_{1}^{\text {Riem }^{3}}=\left[1+39 \beta_{1}-20 \beta_{2}+4 \beta_{3}+28 \beta_{4}+108 \beta_{5}+108 \beta_{6}+756 \beta_{7}+5292 \beta_{8}\right] B_{1}^{\mathrm{E}}, \\
& B_{2}^{\text {Riem }^{3}}=\left[1+7 \beta_{1}-20 \beta_{2}+68 \beta_{3}+476 \beta_{4}+108 \beta_{5}+108 \beta_{6}+756 \beta_{7}+5292 \beta_{8}\right] B_{2}^{\mathrm{E}}, \\
& B_{3}^{\text {Riem }^{3}}=\left[1+39 \beta_{1}-84 \beta_{2}-60 \beta_{3}-420 \beta_{4}+108 \beta_{5}+108 \beta_{6}+756 \beta_{7}+5292 \beta_{8}\right] B_{3}^{\mathrm{E}} .
\end{aligned}
$$

We can check, at this order, which theories satisfy the $3 B_{3}-\left(B_{2}-B_{1} / 2\right)=0$ condition. Evaluating the quantity in the left-hand side, one obtains

$$
3 B_{3}-\left(B_{2}-B_{1} / 2\right)=-\frac{\left(\beta_{1}+2 \beta_{2}\right) L_{\star}^{2}}{32 \pi G} .
$$

Hence, such a combination vanishes for all theories for which $\beta_{1}=-2 \beta_{2}$. This includes, in particular, the cubic Lovelock density, in agreement with the result of [66]. The explicit expressions for the quadratic and cubic theories read

$$
\begin{array}{lll}
B_{1}^{\mathcal{X}_{4}}=\left[1-\frac{104}{3} \lambda_{2}\right] B_{1}^{\mathrm{E}}, & B_{2}^{\mathcal{X}_{4}}=\left[1-\frac{136}{3} \lambda_{2}\right] B_{2}^{\mathrm{E}}, & B_{3}^{\mathcal{X}_{4}}=\left[1-24 \lambda_{2}\right] B_{3}^{\mathrm{E}}, \\
B_{1}^{\mathcal{X}_{6}}=\left[1+136 \lambda_{3}\right] B_{1}^{\mathrm{E}}, & B_{2}^{\mathcal{X}_{6}}=\left[1+200 \lambda_{3}\right] B_{2}^{\mathrm{E}}, & B_{3}^{\mathcal{X}_{6}}=\left[1+72 \lambda_{3}\right] B_{3}^{\mathrm{E}} .
\end{array}
$$

\subsection{Corner regions}

In this subsection we construct the universal function characteristic of corner regions for general holographic cubic gravities using the perturbative HEE functionals. We show that the introduction of such terms in the bulk Lagrangian modifies the angular dependence of the Einstein gravity function, as opposed to previously considered quadratic and $f(R)$ theories. We compute the new functions explicitly and perform some comparisons with the analogous ones corresponding to free scalars and fermions.

General aspects of corner entanglement. The structure of divergences and universal terms in the entanglement entropy gets modified when the entangling surface $\partial A$ contains geometric singularities - see e.g., [86, 87] for some general accounts of this phenomenon in various dimensions. Here, we will focus on the prototypical example of (straight) corners 
in $d=3$ CFTs. Given a fixed time slice, the entanglement entropy corresponding to a corner region of opening angle $\theta$ in the ground state of a CFT regulated by a UV cutoff $\delta$ takes the form

$$
S_{\mathrm{EE}}=b_{1} \frac{H}{\delta}-a(\theta) \log (H / \delta)+b_{0} .
$$

Here, $H$ is an IR regulator and $b_{1}$ is a non-universal coefficient. On the other hand, $b_{0}$ is a coefficient which generically contains a universal non-local contribution and a non-universal part of intrinsically local nature induced by possible redefinitions of the regulator $\delta$.

With respect to the case of smooth regions, the novelty here is the appearance of a new logarithmic divergence controlled by the corner function $a(\theta)$, of universal nature. By now, many aspects of this function have been studied in a plethora of contexts - e.g., for free fields [88-94], for large- $N$ vector models [95], for holographic theories [19, 65, 73, 96-106], in interacting lattice models [107-114], and for general CFTs [18, 115-118]. As a result of this thorough study, the function $a(\theta)$ has been shown to satisfy a number of properties, universal relations and bounds which we summarize now.

On the one hand, the purity of the ground state, which implies the well-known relation $S_{\mathrm{EE}}(A)=S_{\mathrm{EE}}(\bar{A})$, requires $a(\theta)=a(2 \pi-\theta)$. Besides, using strong subadditivity and Lorentz invariance one can show that [89]

$$
a(\theta) \geq 0, \quad \partial_{\theta} a(\theta) \leq 0, \quad \partial_{\theta}^{2} a(\theta) \geq-\frac{\partial_{\theta} a(\theta)}{\sin \theta}, \quad \text { for } \quad \theta \in[0, \pi] .
$$

In particular, this implies that $a(\theta)$ is a positive, monotonously-decreasing and convex function of the opening angle as we vary it from $\theta \sim 0$, corresponding to a very sharp corner, to $\theta \sim \pi$, corresponding to a very open, almost-smooth, corner. In those two limits, the function behaves, respectively, as [88-90]

$$
a(\theta \simeq 0)=\frac{\kappa}{\theta}+\mathcal{O}(\theta), \quad a(\theta \simeq \pi)=\sigma \cdot(\theta-\pi)^{2}+\sum_{p=2} \sigma^{(p-1)} \cdot(\theta-\pi)^{2 p} .
$$

In the first expression, $\kappa$ is a constant which can be shown to coincide with the slab coefficient $\kappa^{(3)}$ - see eq. (5.22) above - for general theories $[65,86]$. In the second formula, we have made manifest the fact that only even powers appear in the expansion. The leading coefficient, $\sigma$, turns out to be related to the stress-energy tensor two-point function coefficient $C_{T}$ through

$$
\sigma=\frac{\pi^{2}}{24} C_{T}
$$

for general CFTs. This relation was conjectured in [18] based on holographic and free-field calculations and proved in full generality in [115] — see also [19, 94, 119] for intermediate progress and partial proofs. In fact, the full corner functions of all CFTs considered so far in the literature turn out to become very close to each other when normalized by $C_{T}$ [18].

Using eq. (5.93) and the third relation in eq. (5.91), a lower bound on $a(\theta)$ valid for general CFTs was obtained in [116]. This takes the form

$$
a(\theta) \geq \mathfrak{a}_{\min }(\theta), \quad \text { where } \quad \mathfrak{a}_{\min }(\theta) \equiv \frac{\pi^{2} C_{T}}{3} \log [1 / \sin (\theta / 2)]
$$


where $C_{T}$ is to be understood as the one corresponding to the theory we are comparing with. The bound turns out to be pretty tight for all theories considered so far, even for considerably small values of the opening angle [116] — see also [120]. In particular, the actual values found from numerical and lattice simulations corresponding to various models for $\theta=\pi / 2$, all fall within the approximate range $a(\pi / 2) / C_{T} \in(1.2,1.3)$ [108-110, 114], whereas the bound value reads $\mathfrak{a}_{\min }(\pi / 2) / C_{T} \simeq 1.1402$.

Additional lower bounds valid also for the general Rényi entropy versions of $a(\theta)$ can be constructed using the inequalities

$$
\operatorname{det}\left\{\partial_{\theta}^{j+k+2} a_{n}(\theta)\right\}_{j, k=0}^{M-1} \geq 0
$$

which follow from the reflection positivity property of Euclidean QFTs [121]. Such bounds were explored in $[114,116]$ and suggest, in particular, that all coefficients in the almostsmooth expansion in eq. (5.92) are positive, i.e., $\sigma^{(p-1)}>0 \forall p .{ }^{21}$ In fact, for sufficiently large $p$, it was observed in [116] that those coefficients behave as

$$
\sigma^{(p)} \simeq \frac{2 \kappa}{\pi^{2 p+3}}, \quad p \gg 1,
$$

where $\kappa$ is the sharp-limit coefficient.

The results mentioned so far are valid for general CFTs. Theories for which $a(\theta)$ has been actually computed for general values of the opening angle are nonetheless scarce. For free scalars and fermions, $a(\theta)$ was obtained numerically from a complicated set of coupled differential and algebraic equations in [88-90]. In addition, the Ryu-Takayanagi prescription allowed for the computation of the corresponding corner function for holographic theories dual to Einstein gravity [96]. The resulting expression is shown below in eq. (5.113) and is given implicitly in terms of two integrals. The only two cases for which a completely explicit expression for $a(\theta)$ is known correspond, respectively, to certain Lifshitz quantum critical points [122] and the so-called "Extensive Mutual Information model" [123-125]. The corresponding corner functions read

$$
a_{\text {Lif. }}(\theta)=\frac{(\theta-\pi)^{2}}{\theta(2 \pi-\theta)}, \quad a_{\text {EMI }}(\theta)=1+(\pi-\theta) \cot \theta .
$$

Using these two functions, it is possible to construct a simple approximation to the corner function of any CFT provided one knows the values of the corresponding sharp and smooth coefficients, $\kappa$ and $\sigma$. This is given by [91]

$$
\tilde{a}(\theta)=\frac{2 \pi(\kappa-3 \pi \sigma)}{\pi^{2}-6} \frac{(\theta-\pi)^{2}}{\theta(2 \pi-\theta)}-\frac{3\left(2 \kappa-\pi^{3} \sigma\right)}{\pi\left(\pi^{2}-6\right)}[1+(\pi-\theta) \cot \theta] .
$$

This respects the asymptotic behavior both as $\theta \rightarrow 0$ and as $\theta \rightarrow \pi$ and produces very precise approximations to the actual free-field and Einstein gravity results. In all cases, the relative agreement is always better than $99 \%$ for all values of $\theta$. If access to some of the subleading coefficients $\sigma^{(p)}$ is also available, improved ansatze can be constructed, as shown in [114].

\footnotetext{
${ }^{21}$ This has been shown to be true in general for $p=1,2,3,4,5$ in [116].
} 
Einstein gravity. Let us quickly review how the corner function is obtained for Einstein gravity $[96,126]$. First, it is useful to write the $\mathrm{AdS}_{3}$ metric as

$$
\mathrm{d} s^{2}=\frac{L_{\star}^{2}}{z^{2}}\left[\mathrm{~d} \tau^{2}+\mathrm{d} z^{2}+\mathrm{d} r^{2}+r^{2} \mathrm{~d} \phi^{2}\right] .
$$

The corner region is defined by $\tau=0, r \geq 0,|\phi| \leq \theta / 2$. We can parametrize the bulk surface as $z=r h(\phi)$, where $h(\phi)$ is a function satisfying $h(\phi \rightarrow \pm \theta / 2) \rightarrow 0$. Unit normals to the surface are given by

$$
n_{1}=\frac{z}{L_{\star}} \partial_{\tau}, \quad n_{2}=\frac{z}{L_{\star} \sqrt{1+h^{2}+\dot{h}^{2}}}\left[\partial_{z}-h \partial_{r}-\frac{\dot{h}}{r} \partial_{\phi}\right] .
$$

Using these we have

$$
\begin{aligned}
h_{\mu \nu} \mathrm{d} x^{\mu} \mathrm{d} x^{\nu}=\frac{L_{\star}^{2}}{z^{2}} & {\left[\mathrm{~d} \tau^{2}+\frac{1}{\left(1+h^{2}+\dot{h}^{2}\right)}\left[\left(h^{2}+\dot{h}^{2}\right) \mathrm{d} z^{2}+\left(1+\dot{h}^{2}\right) \mathrm{d} r^{2}\right.\right.} \\
& \left.\left.+r^{2}\left(1+h^{2}\right) \mathrm{d} \phi^{2}+2 h \mathrm{~d} z \mathrm{~d} r+2 r \dot{h} \mathrm{~d} z \mathrm{~d} \phi-2 r h \dot{h} \mathrm{~d} r \mathrm{~d} \phi\right]\right] .
\end{aligned}
$$

Projectors on the surface are given by

$$
t_{r}=h \partial_{z}+\partial_{r}, \quad t_{\phi}=r \dot{h} \partial_{z}+\partial_{\phi},
$$

and the projected induced metric reads

$$
h_{i j} \mathrm{~d} y^{i} \mathrm{~d} y^{j}=\frac{L_{\star}^{2}}{r^{2} h^{2}}\left[\left(1+h^{2}\right) \mathrm{d} r^{2}+r^{2}\left(1+\dot{h}^{2}\right) \mathrm{d} \phi^{2}+2 r h \dot{h} \mathrm{~d} r \mathrm{~d} \phi\right] .
$$

The non-vanishing components of the extrinsic curvatures, $K_{i j}^{2}$, are in turn given by

$$
\begin{aligned}
K_{r r}^{2} & =\frac{-L_{\star}\left(1+h^{2}\right)}{r^{2} h^{2} \sqrt{1+h^{2}+\dot{h}^{2}}}, \quad K_{r \phi}^{2}=\frac{-L_{\star} \dot{h}}{r h \sqrt{1+h^{2}+\dot{h}^{2}}}, \\
K_{\phi \phi}^{2} & =\frac{-L_{\star}\left(1+h^{2}+\dot{h}^{2}+\ddot{h} h\right)}{h^{2} \sqrt{1+h^{2}+\dot{h}^{2}}} .
\end{aligned}
$$

These are all the pieces we will need to evaluate the corner function for perturbative higherorder gravities.

For our parametrization of the holographic entangling surface, the Ryu-Takayanagi functional becomes

$$
S_{\mathrm{HEE}}^{\mathrm{E}}=\frac{L_{\star}^{2}}{2 G} \int_{\delta / h_{0}}^{H} \frac{\mathrm{d} r}{r} \int_{0}^{\theta / 2-\epsilon} \mathrm{d} \phi \frac{\sqrt{1+h^{2}+\dot{h}^{2}}}{h^{2}},
$$

where we already made manifest the UV cutoff at $z=\delta$ and where $h_{0} \equiv h(0)$ is the maximum value taken by the function $h(\phi)$. Also, the angular cutoff $\epsilon$ is defined through the condition $r h(\theta / 2-\epsilon)=\delta$, which means that the integral over $r$ cannot be performed without doing the angular one first. The extremal surface condition, $K^{2}=0$, reads

$$
2+3 h^{2}+h^{4}+2 \dot{h}^{2}+h\left(1+h^{2}\right) \ddot{h}=0 .
$$


This has a first integral,

$$
\frac{1+h^{2}}{h^{2} \sqrt{1+h^{2}+\dot{h}^{2}}}=\frac{\sqrt{1+h_{0}^{2}}}{h_{0}^{2}},
$$

which can be used to write $\dot{h}$ in terms of $h$ in the RT functional. Trading the integral over $\phi$ by one over $h$ and making the change of variables $y=\sqrt{1 / h^{2}-1 / h_{0}^{2}}$ we are left with

$$
\begin{aligned}
S_{\mathrm{HEE}}^{\mathrm{E}} & =\frac{L_{\star}^{2}}{2 G} \int_{\delta / h_{0}}^{H} \frac{\mathrm{d} r}{r} \int_{0}^{\sqrt{(r / \delta)^{2}-1 / h_{0}^{2}}} \mathrm{~d} y \sqrt{\frac{1+h_{0}^{2}\left(1+y^{2}\right)}{2+h_{0}^{2}\left(1+y^{2}\right)}} \\
& =\frac{L_{\star}^{2}}{2 G} \int_{\delta / h_{0}}^{H} \frac{\mathrm{d} r}{r} \int_{0}^{\infty} \mathrm{d} y\left[\sqrt{\frac{1+h_{0}^{2}\left(1+y^{2}\right)}{2+h_{0}^{2}\left(1+y^{2}\right)}}-1\right]+\frac{L_{\star}^{2}}{2 G} \int_{\delta / h_{0}}^{H} \frac{\mathrm{d} r}{r} \sqrt{\frac{r^{2}}{\delta^{2}}-\frac{1}{h_{0}^{2}}} .
\end{aligned}
$$

Expanding this expression for small $\delta$ one finally obtains

$$
S_{\mathrm{HEE}}^{\mathrm{E}}=\frac{L_{\star}^{2}}{2 G} \frac{H}{\delta}-a_{\mathrm{E}}(\theta) \log (H / \delta)+\mathcal{O}\left(\delta^{0}\right),
$$

in agreement with the general expression eq. (5.90). The result for the Einstein gravity corner function can be written as [96, 126]

$$
\begin{aligned}
a_{\mathrm{E}}(\theta) & =\frac{L_{\star}^{2}}{2 G} \int_{0}^{+\infty} \mathrm{d} y\left[1-\sqrt{\frac{1+h_{0}^{2}\left(1+y^{2}\right)}{2+h_{0}^{2}\left(1+y^{2}\right)}}\right], \\
\theta & =\int_{0}^{h_{0}} \mathrm{~d} h \frac{2 \sqrt{1+h_{0}^{2}} h^{2}}{\sqrt{1+h^{2}} \sqrt{\left(h_{0}^{2}-h^{2}\right)\left(h_{0}^{2}+\left(1+h_{0}^{2}\right) h^{2}\right)}},
\end{aligned}
$$

where the dependence on the opening angle follows implicitly from the relation $h_{0}(\theta)$ determined by the second integral. The above expressions can be alternatively written in terms of elliptic functions [127] as

$$
\begin{aligned}
a_{\mathrm{E}}(\theta) & =\frac{L_{\star}^{2}}{2 G} \sqrt{1+\frac{2}{h_{0}^{2}}}\left[\mathbb{E}\left[\frac{1}{2+h_{0}^{2}}\right]-\frac{\left(1+h_{0}^{2}\right)}{\left(2+h_{0}^{2}\right)} \mathbb{K}\left[\frac{1}{2+h_{0}^{2}}\right]\right], \\
\theta & =-\frac{2 h_{0}}{\sqrt{2+h_{0}^{2}\left(3+h_{0}^{2}\right)}}\left[\mathbb{K}\left[\frac{1}{2+h_{0}^{2}}\right]-\Pi\left[\frac{1+h_{0}^{2}}{2+h_{0}^{2}}, \frac{1}{2+h_{0}^{2}}\right]\right] .
\end{aligned}
$$

It can be verified that $a_{\mathrm{E}}(\theta)$ satisfies all properties explained in the previous subsection. Values of the opening angle close to $\theta=\pi$ correspond to $h_{0} \rightarrow \infty$, and an expansion of the $\theta\left(h_{0}\right)$ integral in that case can be obtained and inverted giving

$$
h_{0}=\left(\frac{\pi}{\pi-\theta}\right)-\frac{3}{4}\left(\frac{\pi-\theta}{\pi}\right)-\frac{11}{64}\left(\frac{\pi-\theta}{\pi}\right)^{3}-\frac{17}{256}\left(\frac{\pi-\theta}{\pi}\right)^{5}-\frac{383}{16384}\left(\frac{\pi-\theta}{\pi}\right)^{7}+\mathcal{O}(\pi-\theta)^{9} .
$$

Inserting this in $a_{\mathrm{E}}(\theta)$ one obtains an expansion of the form of the second expression in eq. (5.92), where the leading smooth-limit coefficients are given by $[65,116]$

$$
\sigma_{\mathrm{E}}=\frac{L_{\star}^{2}}{8 \pi G}, \quad \sigma_{\mathrm{E}}^{\prime}=\frac{5 L_{\star}^{2}}{64 \pi^{3} G}, \quad \sigma_{\mathrm{E}}^{\prime \prime}=\frac{37 L_{\star}^{2}}{512 \pi^{5} G}, \quad \sigma_{\mathrm{E}}^{\prime \prime \prime}=\frac{585 L_{\star}^{2}}{8192 \pi^{7} G}, \quad \sigma_{\mathrm{E}}^{(4)}=\frac{9399 L_{\star}^{2}}{131072 \pi^{9} G} .
$$


As many higher-order coefficients as desired can be determined analytically in the same way. On the other hand, the sharp limit coefficient is given by [65]

$$
\kappa_{\mathrm{E}}=\frac{L_{\star}^{2}}{2 \pi G} \Gamma[3 / 4]^{4} .
$$

Quadratic theories. As observed in [65], the only modification produced on the Einstein gravity corner function $a_{\mathrm{E}}(\theta)$ which arises from including quadratic or $f(R)$ terms in the gravitational action is an overall constant shift. In particular, for an action of the form eq. (4.8) one finds

$$
a_{\operatorname{Riem}^{2}}(\theta)=\left[1-24 \alpha_{1}-6 \alpha_{2}\right] a_{\mathrm{E}}(\theta) .
$$

Hence, no new functional dependence on the opening angle is found from these gravitational interactions. As discussed in some detail in the same paper, the reason for this can be easily understood. On the one hand, all terms involving bulk curvatures will reduce to terms proportional to the Ryu-Takayanagi functional when evaluated on the pure $\mathrm{AdS}_{4}$ background we are considering. On the other hand, any term proportional to $K^{a} K_{a}$ will also be extremized by RT surfaces, since the extremal surface condition reads $K^{a}=0$. As a consequence, terms proportional to $K^{a} K_{a}$ in the action will simply vanish on extremal surfaces and will not contribute. Finally, a term like $K_{a i j} K^{a i j}$ can also be deduced not to contribute from the fact that we can replace the $R_{\mu \nu \rho \sigma} R^{\mu \nu \rho \sigma}$ piece by the Gauss-Bonnet density (plus additional $R^{2}$ and $R_{\mu \nu} R^{\mu \nu}$ terms) whose contribution to the EE functional is the intrinsic Ricci scalar on the RT surface [28, 29], which is a topological term in $(d-1)=2$ dimensions and therefore makes no contribution to the equations of motion. In this case, it does not even modify the Einstein gravity result by an overall constant.

Our results here allow us to compute the corner function for cubic theories and verify that non-trivial modifications of $a_{\mathrm{E}}(\theta)$ arise in the presence of such terms.

Cubic theories. Let us then consider a general cubic action of the form eq. (4.14). If we only turn on the couplings corresponding to $\mathcal{L}_{i}^{(3)}$ with $i=3,4,5,6,7,8$ we find that, similarly to the quadratic case, the corner function is the same as for Einstein gravity up to an overall factor. In the $i=5,6,7,8$ cases, the fact that the functionals have no anomaly contribution imply that the overall coefficient correcting the Einstein gravity result is the same as for $a^{\star(3)}$. For $i=3,4$, even though there is no modification in the functional dependence of the corner function, there is a modification to the overall coefficient coming from the anomaly terms. The result for all these densities reads

$$
a_{\mathcal{L}_{(3,4,5,6,7,8)}^{(3)}}(\theta)=\left[1+6 \beta_{3}+24 \beta_{4}+27 \beta_{5}+27 \beta_{6}+108 \beta_{7}+432 \beta_{8}\right] a_{\mathrm{E}}(\theta)
$$

On the other hand, $\mathcal{L}_{1}^{(3)}$ and $\mathcal{L}_{2}^{(3)}$ do modify the angular dependence of $a_{\mathrm{E}}$. Keeping only those two terms in the action, we find instead

$$
a_{\mathcal{L}_{(1,2)}^{(3)}}(\theta)=\left[1+6 \beta_{1}+12 \beta_{2}\right] a_{\mathrm{E}}(\theta)+\sum_{i=1}^{2} \beta_{i} g_{i}(\theta),
$$


where

$$
\begin{aligned}
& g_{1}(\theta) \equiv+\frac{L_{\star}^{2}}{2 G} \int_{0}^{+\infty} \frac{3\left(1+h_{0}^{2}\right)\left[3+h_{0}^{2}\left(5+4 y^{2}\right)+2 h_{0}^{4}\left(1+y^{2}\right)^{2}\right]}{\left[1+h_{0}^{2}\left(1+y^{2}\right)\right]^{7 / 2} \sqrt{2+h_{0}^{2}\left(1+y^{2}\right)}} \mathrm{d} y, \\
& g_{2}(\theta) \equiv-\frac{L_{\star}^{2}}{2 G} \int_{0}^{+\infty} \frac{6\left(1+h_{0}^{2}\right)\left[3+h_{0}^{2}\left(7+8 y^{2}\right)+4 h_{0}^{4}\left(1+y^{2}\right)^{2}\right]}{\left[1+h_{0}^{2}\left(1+y^{2}\right)\right]^{7 / 2} \sqrt{2+h_{0}^{2}\left(1+y^{2}\right)}} \mathrm{d} y .
\end{aligned}
$$

Hence, at cubic order we find the first examples of holographic corner functions which modify the angular dependence of $a(\theta)$ in a nontrivial way with respect to the Einstein gravity case.

As we mentioned earlier, the almost-smooth limit of the corner function is controlled by $C_{T}$ for all CFTs. For cubic theories, the result for this coefficient appears in eq. (5.58) above. In $d=3$ one finds

$$
C_{T}^{\text {Riem }}{ }^{3}=\left[1+12 \beta_{1}-12 \beta_{2}+6 \beta_{3}+24 \beta_{4}+27 \beta_{5}+27 \beta_{6}+108 \beta_{7}+432 \beta_{8}\right] C_{T}^{\mathrm{E}},
$$

where $C_{T}^{\mathrm{E}}=3 L^{2} /\left(\pi^{3} G\right)$. Now, including all cubic terms in the action, we find for the smooth limit of $a_{\mathrm{Riem}^{3}}(\theta)$ that indeed

$$
\sigma_{\mathrm{Riem}^{3}}=\frac{\pi^{2}}{24} C_{T}^{\mathrm{Riem}^{3}}
$$

holds, as expected. This was in fact previously verified in [19], where several general results regarding the behavior of $a(\theta)$ for holographic theories were discussed, including the fact that $\kappa$ is not universally related to $C_{T}$, as opposed to $\sigma$. The subleading coefficients in the smooth-limit expansion are modified with respect to the Einstein gravity result in an obvious way for $\mathcal{L}_{(3,4,5,6,7,8)}^{(3)}$ but in a nontrivial one for $\mathcal{L}_{1}^{(3)}$ and $\mathcal{L}_{2}^{(3)}$. The first few of them read

$$
\begin{array}{rlrl}
\sigma_{\mathcal{L}_{(1,2)}^{(3)}} & =\left[1+12 \beta_{1}-12 \beta_{2}\right] \sigma_{\mathrm{E}}, & & \sigma_{\mathcal{L}_{(1,2)}^{(3)}}^{\prime}=\left[1+15 \beta_{1}-6 \beta_{2}\right] \sigma_{\mathrm{E}}^{\prime}, \\
\sigma_{\mathcal{L}_{(1,2)}^{(3)}}^{\prime \prime}=\left[1+\frac{1173}{74} \beta_{1}-\frac{189}{37} \beta_{2}\right] \sigma_{\mathrm{E}}^{\prime \prime}, & \sigma_{\mathcal{L}_{(1,2)}^{\prime \prime \prime}}^{(3)}=\left[1+\frac{963}{65} \beta_{1}-\frac{414}{65} \beta_{2}\right] \sigma_{\mathrm{E}}^{\prime \prime \prime}, \\
\sigma_{\mathcal{L}_{(1,2)}^{(4)}}^{(3)}=\left[1+\frac{43946}{3133} \beta_{1}-\frac{24896}{3133} \beta_{2}\right] \sigma_{\mathrm{E}}^{(4)} . &
\end{array}
$$

Just like $\sigma$ is controlled by the stress-tensor two-point coefficient $C_{T}$ for general theories, it is tempting to speculate that $\sigma^{\prime}$ may be controlled by the stress-tensor three-point coefficients, which for $d=3$ CFTs can be chosen to be $C_{T}$ and an additional dimensionless coefficient, customarily denoted $t_{4}$ [128]. This possibility was pointed out in [19] and explored in [116]. There, using the available results for free fields and holographic Einstein gravity it was shown that $\sigma^{\prime}$ was not a linear combination of $C_{T}$ and $C_{T} t_{4}$ in general. Using the results obtained in [129] for $t_{4}$ for general cubic higher-curvature theories, we verify that this is not the case either for this class of theories. In the opposite limit, we find

$$
\kappa_{\text {Riem }^{3}}=\left[1+\frac{69}{5} \beta_{1}-\frac{42}{5} \beta_{2}+6 \beta_{3}+24 \beta_{4}+27 \beta_{5}+27 \beta_{6}+108 \beta_{7}+432 \beta_{8}\right] \kappa_{\mathrm{E}} .
$$


Obviously, the coefficients for $\mathcal{L}_{i}^{(3)}$ with $i=3, \ldots, 8$ are the same as those appearing in $\sigma_{\mathrm{Riem}^{3}}$, but that is not the case for $\mathcal{L}_{1}^{(3)}$ and $\mathcal{L}_{2}^{(3)}$. On the other hand, as expected on general grounds $[65,86], \kappa_{\text {Riem }^{3}}$ matches the coefficient of the slab EE computed above compare with eq. (5.39) for $d=3$.

We would like to perform some more comparisons of our new corner functions. For the sake of conciseness, from now on we restrict the discussion to Einsteinian cubic gravity, whose Lagrangian we introduced in eq. (4.27). We find the corner function for this theory to be given by

$$
a_{\mathrm{ECG}}(\theta)=(1+3 \mu) a_{\mathrm{E}}(\theta)-\frac{\mu L_{\star}^{2}}{2 G} \int_{0}^{\infty} \mathrm{d} y \frac{3\left(1+h_{0}^{2}\right)\left(15+8 h_{0}^{4}\left(1+y^{2}\right)^{2}+h_{0}^{2}\left(23+16 y^{2}\right)\right)}{4\left(1+h_{0}^{2}\left(1+y^{2}\right)\right)^{7 / 2} \sqrt{2+h_{0}^{2}\left(1+y^{2}\right)}} .
$$

This can be also written in terms of elliptic functions as

$$
\begin{array}{r}
a_{\mathrm{ECG}}(\theta)=(1+3 \mu) a_{\mathrm{E}}(\theta)-\frac{\mu L_{\star}^{2}}{40 G h_{0} \sqrt{1+h_{0}^{2}}}\left[\left(-51-51 h_{0}^{2}+8 h_{0}^{4}\right) \mathbb{E}\left[-\frac{1}{1+h_{0}^{2}}\right]\right. \\
\left.+\left(51+47 h_{0}^{2}-8 h_{0}^{4}\right) \mathbb{K}\left[-\frac{1}{1+h_{0}^{2}}\right]\right] .
\end{array}
$$

The first smooth-limit coefficients and the sharp-limit one read in this case

$$
\begin{array}{rlrl}
\sigma_{\mathrm{ECG}} & =[1-3 \mu] \sigma_{\mathrm{E}}, & \sigma_{\mathrm{ECG}}^{\prime} & =\left[1-\frac{33}{4} \mu\right] \sigma_{\mathrm{E}}^{\prime}, \\
\sigma_{\mathrm{ECG}}^{\prime \prime} & =\left[1-\frac{2673}{296} \mu\right] \sigma_{\mathrm{E}}^{\prime \prime}, & \sigma_{\mathrm{ECG}}^{\prime \prime \prime} & =\left[1-\frac{2061}{260} \mu\right] \sigma_{\mathrm{E}}^{\prime \prime \prime}, \\
\sigma_{\mathrm{ECG}}^{(4)} & =\left[1-\frac{41023}{6266} \mu\right] \sigma_{\mathrm{E}}^{(4)}, & \kappa_{\mathrm{ECG}}=\left[1-\frac{123}{20} \mu\right] \kappa_{\mathrm{E}} .
\end{array}
$$

The positivity of these coefficients impose the bound $\mu \leq 0.1107$ (coming from $\sigma_{\mathrm{ECG}}^{\prime \prime} \geq 0$ ). However, as shown in [9], the general bounds on the stress-tensor three-point function coefficient $-4 \leq t_{4} \leq 4$ [7] impose more severe constraints on the allowed values of $\mu$, namely, $-0.00322 \leq \mu \leq 0.00312$. In the perturbative analysis performed in the present paper, bounds on finite values of $\mu$ are not so relevant, but we can use them to give us an idea of how much it is sensible to deviate $\mu$ from zero when performing comparisons with other theories. In figure 1 we have plotted $a_{\mathrm{ECG}}(\theta)$ for the limiting values $\mu \simeq-0.00322$ and $\mu \simeq 0.00312$ (all intermediate values of $\mu$ lie between the two curves) along with the Einstein gravity result and the free scalar $\left(t_{4}=+4\right)$ and free fermion $\left(t_{4}=-4\right)$ ones [88-90]. We can see that all curves are remarkably close to each other, in agreement with the observation/conjecture of [18] that $a(\theta) / C_{T}$ is an almost-universal quantity for general CFTs. We observe this to be the case for the whole family of theories parametrized by the continuous parameter $\mu$ lying between the limiting cases extremizing the value of $t_{4}$. By making the values of $|\mu|$ greater, we can obtain curves which deviate more significantly from the Einstein and free-field curves (see dotted lines in figure 1). However, those would correspond to toy models of CFTs which do not respect the general bounds $\left|t_{4}\right| \leq 4$. Hence, 

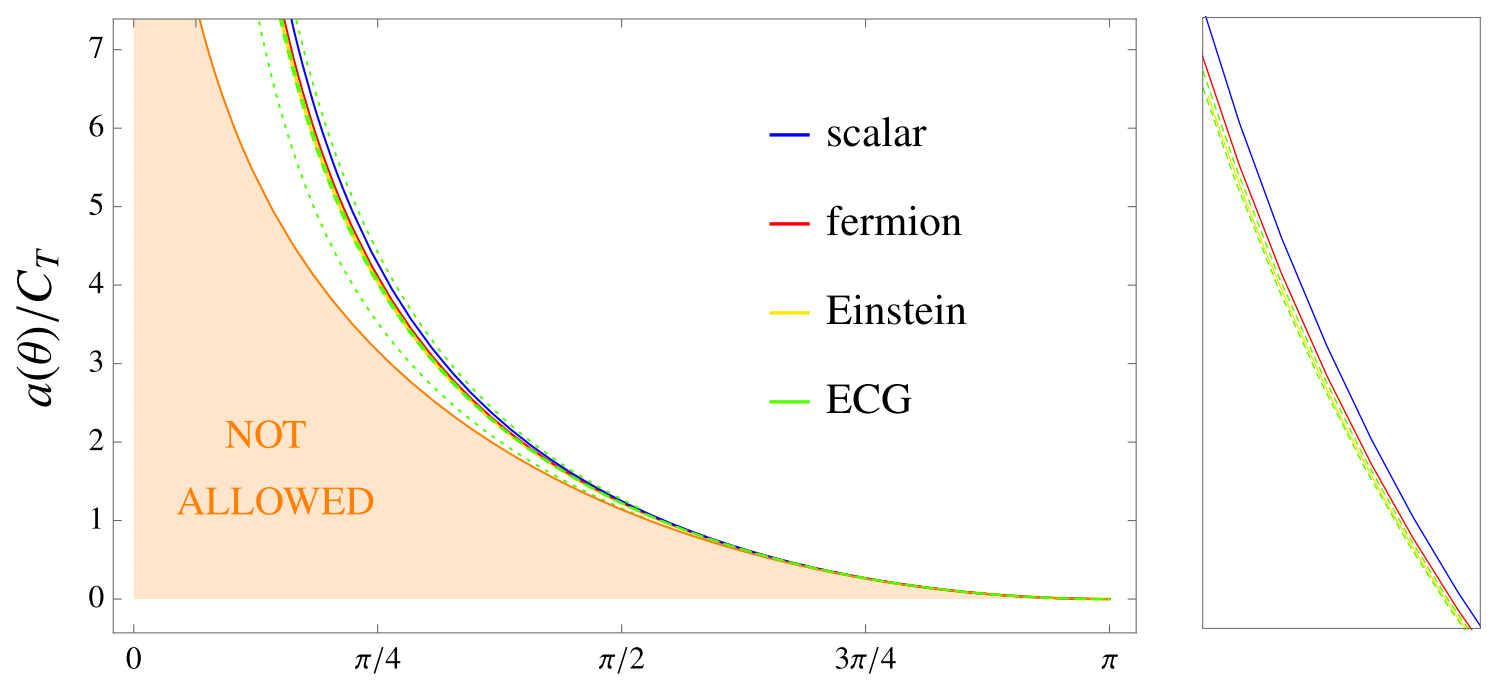

Figure 1. We plot the corner functions (normalized by their respective charges $C_{T}$ ) for a free scalar (blue), a free fermion (red), holographic Einstein gravity (yellow) and holographic Einsteinian cubic gravity (green). For the limit value $\mu \simeq 0.00312$ corresponding to $t_{4}=+4$ (see discussion below), the curve lies very close but slightly below the Einstein gravity result (green dashed line). The case $\mu \simeq-0.00322$ corresponding to the other limit value $\left(t_{4}=-4\right)$ lies even closer but slightly above the Einstein gravity curve and just below the fermion one. The right plot is a zoom of the curves between $\theta=\pi / 4$ and $\theta=3 \pi / 8$. The orange region in the left plot is excluded for general theories by the inequality eq. (5.94). The green dotted curves correspond to the values $\mu=-0.05$ (upper curve) and $\mu=+0.05$ (lower curve) which we have included (only) in the left plot for visual reference.

it is reasonable to expect that for actual CFTs the curves will indeed fall extremely close to each other in general. In fact, the ECG curves with $t_{4}=4$ and $t_{4}=-4$ lie even closer to the Einstein gravity one than the scalar and fermion curves do. This suggests that the scalar field curve may be an upper bound for general CFTs.

On the other hand, the possibility that the Einstein gravity curve is a lower bound for general curves suggested in [18] seems to be ruled out by our analysis: the introduction of higher-curvature corrections allows to go below the Einstein gravity one. ${ }^{22}$ Note that such conjecture was also supported by the fact that while $t_{4}=0$ for Einstein gravity, both the scalar and the fermion curves - which have, respectively, the largest positive and negative values of $t_{4}$ allowed - lie above it. Here we observe that, contrary to the scalar case, ECG theories with $t_{4} \geq 0$ lie below the Einstein gravity one.

In the previous subsection, we mentioned the possibility of approximating the function $a(\theta)$ for a given theory using the values of the almost-smooth and very-sharp limit coefficients, $\sigma$ and $\kappa$. The proposed trial function $\tilde{a}(\theta)$ appears in eq. (5.98). We can use the new ECG corner functions to test the accuracy of such approximation beyond the free-field and Einstein cases explored in [91]. In figure 2, we plot $1-a(\theta) / \tilde{a}(\theta)$ for various values of the ECG coupling falling between the limiting cases of $t_{4}= \pm 4$. We observe that in all cases, the error in the approximation never exceeds $\sim 1.2 \%$ for any value of the opening angle, the approximation being slightly better for negative values of $\mu$. This provides good

\footnotetext{
${ }^{22}$ The same conclusion was previously reached in [19].
} 


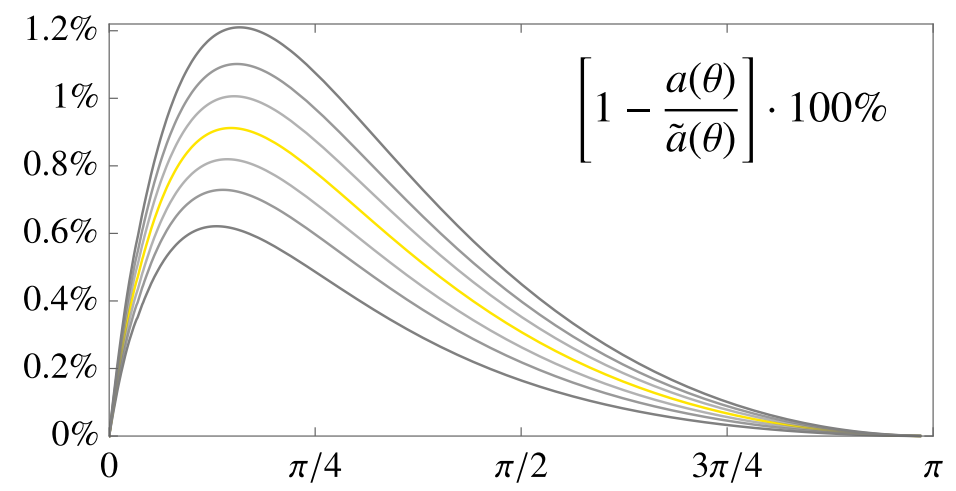

Figure 2. We plot $1-a(\theta) / \tilde{a}(\theta)$ where $a(\theta)$ is the exact corner function and $\tilde{a}(\theta)$ the trial function defined in eq. (5.98) for Einstein gravity (yellow) and ECG for different values of $\mu$ (from top to bottom: $\mu=+0.00312,+0.002,+0.001,-0.001,-0.002,-0.00322)$. The disagreement between both functions is always smaller than $\sim 1.2 \%$ throughout the whole range of values of the opening angle.

evidence that $\tilde{a}(\theta)$ can be used as an accurate approximation to the exact corner function for general CFTs.

\section{Final comments}

The main results of the paper appear summarized in the introduction. Let us conclude with some final comments.

In this paper we have obtained a new formula for the HEE functional valid for general higher-curvature gravities when considered as perturbative corrections to Einstein gravity - the covariant form of the new expression appears in eq. (3.66). This formula, which gets rid of the weighted sum over $\alpha$ present in the original functional (2.4), is computationally much simpler to use in concrete cases beyond cubic order, and allowed us to evaluate the explicit form of the functionals for general quartic densities. If desired, it should be possible to implement it in a mathematical software and compute the analogous expressions for even higher orders.

Besides its computational simplicity, the new form of the anomaly piece can be suggestively written in terms of the exponential of a differential operator - this is particularly neat for Lovelock theories, see eq. (3.40). This form may be useful for potential applications beyond HEE, which may include new versions of the second law for higher-curvature black holes, e.g., along the lines of [130, 131].

As we have emphasized throughout the paper, the fact that our new expression is restricted to perturbative higher-curvature theories beyond quadratic order is related to the splitting problem, which requires the identification of the precise way in which Riemann tensor components must be decomposed into pieces of different weight $q_{\alpha}$ in the original functional for a given theory. While this could be in principle determined using the procedure developed in [43] on a theory by theory basis, ${ }^{23}$ general results can be obtained

\footnotetext{
${ }^{23}$ To the best of our knowledge, this has not been done explicitly for any non-trivial higher-curvature theory yet.
} 
at leading order in the couplings by considering the splittings corresponding to Einstein gravity, which has been our approach in this paper. Nonetheless, we would like to stress that, in fact, our formalism should be straightforwardly adaptable to situations in which the Riemann tensor components split in a different fashion. In that case, instead of the separation into type $A$ and $B$ components one may have to introduce additional types $C$, $D$, etc., depending on the different possible weights corresponding to the different split components. One could even think of a sort of general-splitting version of our formulas.

In section 5 we have used our new expressions for cubic theories to evaluate several universal contributions to the EE characterizing the holographic CFTs they define. An analogous catalogue of coefficients could be obtained for quartic theories using the functionals presented in subsection 4.5. Naturally, there are many possible additional applications within the HEE framework one could consider exploring using the new functionals presented here.

Having studied the perturbative case, it is natural to wonder how difficult it would be to construct functionals valid at second order in the couplings. For theories affected by the splitting problem, this would become considerably more involved. On the one hand, obtaining the right functionals would require identifying the correct splittings for the different Riemann tensor components on a theory-by-theory basis. In addition, once the functionals were available, computing actual entanglement entropies would involve determining the new entangling surfaces by solving the corresponding Euler-Lagrange equations, as the RT ones would no longer be extrema. This would be a challenging task in general, especially given the higher-order nature of the equations in such cases.

\section{Acknowledgments}

We thank Felix Haehl, Rong-Xin Miao, Rob Myers and William Witczak-Krempa for useful discussions on related topics. PB and JC were supported by the Simons Foundation through the "It From Qubit" Simons collaboration. The work of AVL is supported by the Spanish MECD fellowship FPU16/06675, and by MINECO FPA2017-84436-P, Xunta de Galicia ED431C 2017/07, Xunta de Galicia (Centro singular de investigación de Galicia accreditation 2019-2022) and the European Union (European Regional Development Fund-ERDF), "María de Maeztu" Units of Excellence MDM-2016-0692, and the Spanish Research State Agency.

\section{A Proof of identities (3.26) and (3.28)}

In this appendix we present short proofs of the relations eq. (3.26) and eq. (3.28) used in the derivation of the new HEE functional formula.

For the first, we want to show that

$$
\sum_{\lambda=0}^{\tilde{T}} \frac{(-1)^{\lambda}}{(\lambda+n)} \frac{1}{\lambda !(\tilde{T}-\lambda) !}=\frac{(n-1) !}{(\tilde{T}+n) !}
$$


Step by step, we have

$$
\begin{aligned}
\sum_{\lambda=0}^{\tilde{T}} \frac{(-1)^{\lambda}}{(\lambda+n)} \frac{1}{\lambda !(\tilde{T}-\lambda) !} & =\sum_{\lambda=0}^{\tilde{T}} \frac{(-1)^{\lambda}}{(\lambda+n) !(\tilde{T}-\lambda) !}(\lambda+n-1) \cdots(\lambda+1) \\
& =\frac{1}{(\tilde{T}+n) !} \partial_{x}^{n-1}\left[\begin{array}{c}
\tilde{T} \\
\lambda=0
\end{array}\left(\begin{array}{c}
\tilde{T}+n \\
\lambda+n
\end{array}\right)(-1)^{\lambda} x^{\lambda+n-1}\right]_{x=1} \\
& =\frac{1}{(\tilde{T}+n) !} \partial_{x}^{n-1}\left[\sum_{\lambda=n}^{T}\left(\begin{array}{c}
\tilde{T}+n \\
\lambda
\end{array}\right)(-1)^{\lambda-n} x^{\lambda-1}\right]_{x=1} \\
& =\frac{1}{(\tilde{T}+n) !} \partial_{x}^{n-1}\left[\begin{array}{l}
\tilde{T}+n \\
\lambda=n
\end{array}\left(\begin{array}{c}
\tilde{T}+n \\
\lambda
\end{array}\right)(-1)^{\lambda-n} x^{\lambda-1}\right]_{x=1} \\
& =\frac{(-1)^{n}}{(\tilde{T}+n) !} \partial_{x}^{n-1}\left[\frac{1}{x} \sum_{\lambda=0}^{\tilde{T}+n}\left(\begin{array}{c}
\tilde{T}+n \\
\lambda
\end{array}\right)(-x)^{\lambda}-\frac{1}{x}\left(\begin{array}{c}
\tilde{T}+n \\
0
\end{array}\right)\right. \\
& -\frac{(-1)^{n}}{(\tilde{T}+n) !} \partial_{x}^{n-1}\left[\frac{(1-x)^{\tilde{T}+n}}{x}-\frac{1}{x}\right]_{x=1} \\
& =\frac{(n-1) !}{(\tilde{T}+n) !},
\end{aligned}
$$

where the first term inside the brackets does not survive after $(n-1)$ derivatives evaluated at $x=1$ because of the factor $(1-x)^{\tilde{T}+n}$, and we have used:

$$
\partial_{x}^{n-1}\left(\frac{1}{x}\right)=\frac{(-1)^{n-1}(n-1) !}{x^{n}} .
$$

As for the second identity, we want to show that

$$
\sum_{\lambda=0}^{T} \frac{(-1)^{\lambda}}{\lambda !(T-\lambda) !} \frac{(2 \lambda+1) !}{(2 \lambda+m) !}=\frac{1}{T !(m-2) !} \int_{0}^{1} \mathrm{~d} z z\left(1-z^{2}\right)^{T}(1-z)^{m-2}
$$

In hopefully self-evident steps we find

$$
\begin{aligned}
\sum_{\lambda=0}^{T} \frac{(-1)^{\lambda}}{\lambda !(T-\lambda) !} \frac{(2 \lambda+1) !}{(2 \lambda+m) !} & =\sum_{\lambda=0}^{T} \frac{(-1)^{\lambda}}{\lambda !(T-\lambda) !} \frac{1}{(2 \lambda+m) \cdots(2 \lambda+2)} \\
& =\sum_{\lambda=0}^{T} \frac{(-1)^{\lambda}}{\lambda !(T-\lambda) !} \int_{0}^{1} \mathrm{~d} x_{1} \int_{0}^{x_{1}} \mathrm{~d} x_{2} \cdots \int_{0}^{x_{m-2}} \mathrm{~d} x_{m-1} x_{m-1}^{2 \lambda+1} \\
& =\int_{0}^{1} \mathrm{~d} x_{1} \int_{0}^{x_{1}} \mathrm{~d} x_{2} \cdots \int_{0}^{x_{m-2}} \mathrm{~d} x_{m-1} x_{m-1} \sum_{\lambda=0}^{T} \frac{\left(-x_{m-1}^{2}\right)^{\lambda}}{\lambda !(T-\lambda) !} \\
& =\frac{1}{T !} \int_{0}^{1} \mathrm{~d} x_{1} \int_{0}^{x_{1}} \mathrm{~d} x_{2} \cdots \int_{0}^{x_{m-3}} \mathrm{~d} x_{m-2} \int_{0}^{x_{m-2}} \mathrm{~d} z z\left(1-z^{2}\right)^{T}
\end{aligned}
$$


where we have relabelled $x_{m-1} \equiv z$. We can reorder the integrals now using the following identity:

$$
\begin{aligned}
& \int_{0}^{1} \mathrm{~d} x_{1} \int_{0}^{x_{1}} \mathrm{~d} x_{2} \cdots \int_{0}^{x_{m-3}} \mathrm{~d} x_{m-2} \int_{0}^{x_{m-2}} \mathrm{~d} z f(z) \\
& =\int_{0}^{1} \mathrm{~d} z f(z) \int_{z}^{1} \mathrm{~d} x_{1} \int_{z}^{x_{1}} \mathrm{~d} x_{2} \cdots \int_{z}^{x_{m-3}} \mathrm{~d} x_{m-2},
\end{aligned}
$$

and then use

$$
\int_{z}^{1} \mathrm{~d} x_{1} \int_{z}^{x_{1}} \mathrm{~d} x_{2} \cdots \int_{z}^{x_{m-3}} \mathrm{~d} x_{m-2}=\frac{1}{(m-2) !}\left(\int_{z}^{1} \mathrm{~d} x\right)^{m-2}=\frac{(1-z)^{m-2}}{(m-2) !},
$$

to finally obtain eq. (A.6).

Open Access. This article is distributed under the terms of the Creative Commons Attribution License (CC-BY 4.0), which permits any use, distribution and reproduction in any medium, provided the original author(s) and source are credited.

\section{References}

[1] M.T. Grisaru, A.E.M. van de Ven and D. Zanon, Four Loop $\beta$-function for the $N=1$ and $N=2$ Supersymmetric Nonlinear Sigma Model in Two-Dimensions, Phys. Lett. B 173 (1986) 423 [INSPIRE].

[2] D.J. Gross and E. Witten, Superstring Modifications of Einstein's Equations, Nucl. Phys. B 277 (1986) 1 [INSPIRE].

[3] S.S. Gubser, I.R. Klebanov and A.A. Tseytlin, Coupling constant dependence in the thermodynamics of $N=4$ supersymmetric Yang-Mills theory, Nucl. Phys. B 534 (1998) 202 [hep-th/9805156] [INSPIRE].

[4] J.M. Maldacena, The Large $N$ limit of superconformal field theories and supergravity, Int. J. Theor. Phys. 38 (1999) 1113 [hep-th/9711200] [INSPIRE].

[5] E. Witten, Anti-de Sitter space and holography, Adv. Theor. Math. Phys. 2 (1998) 253 [hep-th/9802150] [INSPIRE].

[6] S.S. Gubser, I.R. Klebanov and A.M. Polyakov, Gauge theory correlators from noncritical string theory, Phys. Lett. B 428 (1998) 105 [hep-th/9802109] [INSPIRE].

[7] A. Buchel, J. Escobedo, R.C. Myers, M.F. Paulos, A. Sinha and M. Smolkin, Holographic GB gravity in arbitrary dimensions, JHEP 03 (2010) 111 [arXiv:0911.4257] [INSPIRE].

[8] R.C. Myers, M.F. Paulos and A. Sinha, Holographic studies of quasi-topological gravity, JHEP 08 (2010) 035 [arXiv: 1004.2055] [INSPIRE].

[9] P. Bueno, P.A. Cano and A. Ruipérez, Holographic studies of Einsteinian cubic gravity, JHEP 03 (2018) 150 [arXiv: 1802.00018] [INSPIRE].

[10] X.O. Camanho, J.D. Edelstein and J.M. Sánchez De Santos, Lovelock theory and the AdS/CFT correspondence, Gen. Rel. Grav. 46 (2014) 1637 [arXiv:1309.6483] [INSPIRE].

[11] J. de Boer, M. Kulaxizi and A. Parnachev, Holographic Lovelock Gravities and Black Holes, JHEP 06 (2010) 008 [arXiv:0912.1877] [INSPIRE]. 
[12] R.C. Myers and A. Sinha, Seeing a c-theorem with holography, Phys. Rev. D 82 (2010) 046006 [arXiv: 1006.1263] [INSPIRE].

[13] R.C. Myers and A. Sinha, Holographic c-theorems in arbitrary dimensions, JHEP 01 (2011) 125 [arXiv: 1011.5819] [inSPIRE].

[14] Y. Kats and P. Petrov, Effect of curvature squared corrections in AdS on the viscosity of the dual gauge theory, JHEP 01 (2009) 044 [arXiv:0712.0743] [INSPIRE].

[15] M. Brigante, H. Liu, R.C. Myers, S. Shenker and S. Yaida, Viscosity Bound Violation in Higher Derivative Gravity, Phys. Rev. D 77 (2008) 126006 [arXiv:0712.0805] [InSPIRE].

[16] X.O. Camanho, J.D. Edelstein and M.F. Paulos, Lovelock theories, holography and the fate of the viscosity bound, JHEP 05 (2011) 127 [arXiv:1010.1682] [INSPIRE].

[17] M. Mezei, Entanglement entropy across a deformed sphere, Phys. Rev. D 91 (2015) 045038 [arXiv:1411.7011] [INSPIRE].

[18] P. Bueno, R.C. Myers and W. Witczak-Krempa, Universality of corner entanglement in conformal field theories, Phys. Rev. Lett. 115 (2015) 021602 [arXiv:1505. 04804] [INSPIRE].

[19] R.-X. Miao, A holographic proof of the universality of corner entanglement for CFTs, JHEP 10 (2015) 038 [arXiv: 1507.06283] [INSPIRE].

[20] P. Bueno, P.A. Cano, R.A. Hennigar and R.B. Mann, Universality of Squashed-Sphere Partition Functions, Phys. Rev. Lett. 122 (2019) 071602 [arXiv:1808.02052] [INSPIRE].

[21] P. Bueno, P.A. Cano, R.A. Hennigar, V.A. Penas and A. Ruipérez, Partition functions on slightly squashed spheres and flux parameters, JHEP 04 (2020) 123 [arXiv:2001.10020] [INSPIRE].

[22] S. Ryu and T. Takayanagi, Holographic derivation of entanglement entropy from AdS/CFT, Phys. Rev. Lett. 96 (2006) 181602 [hep-th/0603001] [INSPIRE].

[23] S. Ryu and T. Takayanagi, Aspects of Holographic Entanglement Entropy, JHEP 08 (2006) 045 [hep-th/0605073] [INSPIRE].

[24] J.D. Bekenstein, Black holes and entropy, Phys. Rev. D 7 (1973) 2333 [InSPIRE].

[25] S.W. Hawking, Particle Creation by Black Holes, Commun. Math. Phys. 43 (1975) 199 [Erratum ibid. 46 (1976) 206] [INSPIRE].

[26] R.M. Wald, Black hole entropy is the Noether charge, Phys. Rev. D 48 (1993) R3427 [gr-qc/9307038] [INSPIRE].

[27] V. Iyer and R.M. Wald, Some properties of Noether charge and a proposal for dynamical black hole entropy, Phys. Rev. D 50 (1994) 846 [gr-qc/9403028] [InSPIRE].

[28] L.-Y. Hung, R.C. Myers and M. Smolkin, On Holographic Entanglement Entropy and Higher Curvature Gravity, JHEP 04 (2011) 025 [arXiv:1101.5813] [INSPIRE].

[29] T. Jacobson and R.C. Myers, Black hole entropy and higher curvature interactions, Phys. Rev. Lett. 70 (1993) 3684 [hep-th/9305016] [INSPIRE].

[30] D.V. Fursaev, A. Patrushev and S.N. Solodukhin, Distributional Geometry of Squashed Cones, Phys. Rev. D 88 (2013) 044054 [arXiv:1306.4000] [InSPIRE].

[31] A. Lewkowycz and J. Maldacena, Generalized gravitational entropy, JHEP 08 (2013) 090 [arXiv: 1304.4926] [INSPIRE].

[32] X. Dong, Holographic Entanglement Entropy for General Higher Derivative Gravity, JHEP 01 (2014) 044 [arXiv: 1310.5713] [INSPIRE]. 
[33] J. Camps, Generalized entropy and higher derivative Gravity, JHEP 03 (2014) 070 [arXiv:1310.6659] [INSPIRE].

[34] A. Bhattacharyya, M. Sharma and A. Sinha, On generalized gravitational entropy, squashed cones and holography, JHEP 01 (2014) 021 [arXiv: 1308.5748] [INSPIRE].

[35] A. Bhattacharyya, A. Kaviraj and A. Sinha, Entanglement entropy in higher derivative holography, JHEP 08 (2013) 012 [arXiv:1305.6694] [INSPIRE].

[36] A. Bhattacharyya and M. Sharma, On entanglement entropy functionals in higher derivative gravity theories, JHEP 10 (2014) 130 [arXiv:1405.3511] [INSPIRE].

[37] B. Chen and J.-j. Zhang, Note on generalized gravitational entropy in Lovelock gravity, JHEP 07 (2013) 185 [arXiv: 1305.6767] [InSPIRE].

[38] X. Dong and R.-X. Miao, Generalized Gravitational Entropy from Total Derivative Action, JHEP 12 (2015) 100 [arXiv:1510.04273] [INSPIRE].

[39] J. Harper, M. Headrick and A. Rolph, Bit Threads in Higher Curvature Gravity, JHEP 11 (2018) 168 [arXiv: 1807.04294] [INSPIRE].

[40] Y. Huang and R.-X. Miao, A note on the resolution of the entropy discrepancy, Phys. Lett. $B \mathbf{7 4 9}$ (2015) 489 [arXiv: 1504.02301] [INSPIRE].

[41] A. Ghodsi and M. Moghadassi, Holographic entanglement entropy from minimal surfaces with/without extrinsic curvature, JHEP 02 (2016) 037 [arXiv:1508.02527] [INSPIRE].

[42] R.-X. Miao and W.-z. Guo, Holographic Entanglement Entropy for the Most General Higher Derivative Gravity, JHEP 08 (2015) 031 [arXiv:1411.5579] [INSPIRE].

[43] X. Dong and A. Lewkowycz, Entropy, Extremality, Euclidean Variations, and the Equations of Motion, JHEP 01 (2018) 081 [arXiv: 1705.08453] [INSPIRE].

[44] J. Camps and W.R. Kelly, Generalized gravitational entropy without replica symmetry, JHEP 03 (2015) 061 [arXiv: 1412.4093] [INSPIRE].

[45] R.-X. Miao, Universal Terms of Entanglement Entropy for 6d CFTs, JHEP 10 (2015) 049 [arXiv: 1503.05538] [INSPIRE].

[46] J. Camps, Gravity duals of boundary cones, JHEP 09 (2016) 139 [arXiv:1605.08588] [INSPIRE].

[47] D. Lovelock, Divergence-free tensorial concomitants, Aequat. Math. 4 (1970) 127.

[48] D. Lovelock, The Einstein tensor and its generalizations, J. Math. Phys. 12 (1971) 498 [INSPIRE].

[49] T. Padmanabhan and D. Kothawala, Lanczos-Lovelock models of gravity, Phys. Rept. 531 (2013) 115 [arXiv: 1302.2151] [INSPIRE].

[50] E. Cáceres, R. Castillo Vásquez and A. Vilar López, Entanglement entropy in cubic gravitational theories, arXiv:2009.11595 [INSPIRE].

[51] J. Oliva and S. Ray, A new cubic theory of gravity in five dimensions: Black hole, Birkhoff's theorem and C-function, Class. Quant. Grav. 27 (2010) 225002 [arXiv:1003.4773] [INSPIRE].

[52] R.C. Myers and B. Robinson, Black Holes in Quasi-topological Gravity, JHEP 08 (2010) 067 [arXiv: 1003.5357] [INSPIRE].

[53] J. Oliva and S. Ray, Birkhoff's Theorem in Higher Derivative Theories of Gravity, Class. Quant. Grav. 28 (2011) 175007 [arXiv:1104.1205] [INSPIRE]. 
[54] J. Oliva and S. Ray, Birkhoff's Theorem in Higher Derivative Theories of Gravity II, Phys. Rev. D 86 (2012) 084014 [arXiv: 1201.5601] [INSPIRE].

[55] P. Bueno and P.A. Cano, Einsteinian cubic gravity, Phys. Rev. D 94 (2016) 104005 [arXiv: 1607.06463] [INSPIRE].

[56] R.A. Hennigar and R.B. Mann, Black holes in Einsteinian cubic gravity, Phys. Rev. D 95 (2017) 064055 [arXiv: 1610.06675] [INSPIRE].

[57] P. Bueno and P.A. Cano, Four-dimensional black holes in Einsteinian cubic gravity, Phys. Rev. D 94 (2016) 124051 [arXiv:1610.08019] [INSPIRE].

[58] M. Mir, R.A. Hennigar, J. Ahmed and R.B. Mann, Black hole chemistry and holography in generalized quasi-topological gravity, JHEP 08 (2019) 068 [arXiv:1902.02005] [INSPIRE].

[59] R.A. Hennigar, D. Kubizňák and R.B. Mann, Generalized quasitopological gravity, Phys. Rev. D 95 (2017) 104042 [arXiv:1703.01631] [INSPIRE].

[60] P. Bueno and P.A. Cano, On black holes in higher-derivative gravities, Class. Quant. Grav. 34 (2017) 175008 [arXiv: 1703.04625] [INSPIRE].

[61] P. Bueno, P.A. Cano and R.A. Hennigar, (Generalized) quasi-topological gravities at all orders, Class. Quant. Grav. 37 (2020) 015002 [arXiv: 1909.07983] [INSPIRE].

[62] S.A. Fulling, R.C. King, B.G. Wybourne and C.J. Cummins, Normal forms for tensor polynomials. 1: The Riemann tensor, Class. Quant. Grav. 9 (1992) 1151 [INSPIRE].

[63] P. Bueno, P.A. Cano, V.S. Min and M.R. Visser, Aspects of general higher-order gravities, Phys. Rev. D 95 (2017) 044010 [arXiv:1610.08519] [InSPIRE].

[64] J. de Boer, M. Kulaxizi and A. Parnachev, Holographic Entanglement Entropy in Lovelock Gravities, JHEP 07 (2011) 109 [arXiv:1101.5781] [InSPIRE].

[65] P. Bueno and R.C. Myers, Corner contributions to holographic entanglement entropy, JHEP 08 (2015) 068 [arXiv: 1505.07842] [INSPIRE].

[66] B.R. Safdi, Exact and Numerical Results on Entanglement Entropy in (5+1)-Dimensional CFT, JHEP 12 (2012) 005 [arXiv:1206.5025] [INSPIRE].

[67] P.A. Cano, Lovelock action with nonsmooth boundaries, Phys. Rev. D 97 (2018) 104048 [arXiv:1803.00172] [INSPIRE].

[68] C. Imbimbo, A. Schwimmer, S. Theisen and S. Yankielowicz, Diffeomorphisms and holographic anomalies, Class. Quant. Grav. 17 (2000) 1129 [hep-th/9910267] [INSPIRE].

[69] A. Schwimmer and S. Theisen, Entanglement Entropy, Trace Anomalies and Holography, Nucl. Phys. B 801 (2008) 1 [arXiv:0802.1017] [InSPIRE].

[70] S.N. Solodukhin, Entanglement entropy, conformal invariance and extrinsic geometry, Phys. Lett. B 665 (2008) 305 [arXiv:0802.3117] [INSPIRE].

[71] D.V. Fursaev, Entanglement Renyi Entropies in Conformal Field Theories and Holography, JHEP 05 (2012) 080 [arXiv:1201.1702] [INSPIRE].

[72] H. Casini, M. Huerta and R.C. Myers, Towards a derivation of holographic entanglement entropy, JHEP 05 (2011) 036 [arXiv:1102.0440] [INSPIRE].

[73] P. Fonda, D. Seminara and E. Tonni, On shape dependence of holographic entanglement entropy in $A d S_{4} / C F T_{3}, J H E P 12$ (2015) 037 [arXiv: 1510.03664] [INSPIRE]. 
[74] G. Anastasiou, J. Moreno, R. Olea and D. Rivera-Betancour, Shape dependence of renormalized holographic entanglement entropy, JHEP 09 (2020) 173 [arXiv:2002.06111] [INSPIRE].

[75] M.J. Duff, Observations on Conformal Anomalies, Nucl. Phys. B 125 (1977) 334 [InSPIRE].

[76] H. Osborn and A.C. Petkou, Implications of conformal invariance in field theories for general dimensions, Annals Phys. 231 (1994) 311 [hep-th/9307010] [INSPIRE].

[77] B. Tekin, Particle Content of Quadratic and $f\left(R_{\mu \nu \sigma \rho}\right)$ Theories in $(A) d S$, Phys. Rev. D 93 (2016) 101502 [arXiv: 1604.00891] [INSPIRE].

[78] L. Bonora, P. Pasti and M. Bregola, Weyl COCYCLES, Class. Quant. Grav. 3 (1986) 635 [inSPIRE].

[79] S. Deser and A. Schwimmer, Geometric classification of conformal anomalies in arbitrary dimensions, Phys. Lett. B 309 (1993) 279 [hep-th/9302047] [INSPIRE].

[80] M. Henningson and K. Skenderis, The Holographic Weyl anomaly, JHEP 07 (1998) 023 [hep-th/9806087] [INSPIRE].

[81] F. Bastianelli, S. Frolov and A.A. Tseytlin, Conformal anomaly of (2,0) tensor multiplet in six-dimensions and AdS/CFT correspondence, JHEP 02 (2000) 013 [hep-th/0001041] [INSPIRE].

[82] J. de Boer, M. Kulaxizi and A. Parnachev, $A d S_{7} / C F T_{6}$, Gauss-Bonnet Gravity, and Viscosity Bound, JHEP 03 (2010) 087 [arXiv:0910.5347] [InSPIRE].

[83] R.-X. Miao, A Note on Holographic Weyl Anomaly and Entanglement Entropy, Class. Quant. Grav. 31 (2014) 065009 [arXiv:1309.0211] [InSPIRE].

[84] H. Lü and C.N. Pope, Critical Gravity in Four Dimensions, Phys. Rev. Lett. 106 (2011) 181302 [arXiv: 1101.1971] [INSPIRE].

[85] S. Deser, H. Liu, H. Lü, C.N. Pope, T.C. Sisman and B. Tekin, Critical Points of D-Dimensional Extended Gravities, Phys. Rev. D 83 (2011) 061502 [arXiv:1101.4009] [INSPIRE].

[86] R.C. Myers and A. Singh, Entanglement Entropy for Singular Surfaces, JHEP 09 (2012) 013 [arXiv: 1206.5225] [INSPIRE].

[87] P. Bueno, H. Casini and W. Witczak-Krempa, Generalizing the entanglement entropy of singular regions in conformal field theories, JHEP 08 (2019) 069 [arXiv:1904.11495] [INSPIRE].

[88] H. Casini and M. Huerta, Universal terms for the entanglement entropy in $2+1$ dimensions, Nucl. Phys. B 764 (2007) 183 [hep-th/0606256] [InSPIRE].

[89] H. Casini, M. Huerta and L. Leitao, Entanglement entropy for a Dirac fermion in three dimensions: Vertex contribution, Nucl. Phys. B 814 (2009) 594 [arXiv:0811.1968] [INSPIRE].

[90] H. Casini and M. Huerta, Entanglement entropy in free quantum field theory, J. Phys. A 42 (2009) 504007 [arXiv:0905.2562] [INSPIRE].

[91] P. Bueno, R.C. Myers and W. Witczak-Krempa, Universal corner entanglement from twist operators, JHEP 09 (2015) 091 [arXiv: 1507.06997] [INSPIRE].

[92] J.S. Dowker, Conformal weights of charged Renyi entropy twist operators for free Dirac fields in arbitrary dimensions, arXiv:1510.08378 [INSPIRE]. 
[93] J.S. Dowker, Note on Renyi vertex contributions and twist operator weights for free scalar fields, arXiv: 1509.00782 [INSPIRE].

[94] H. Elvang and M. Hadjiantonis, Exact results for corner contributions to the entanglement entropy and Rényi entropies of free bosons and fermions in 3d, Phys. Lett. B 749 (2015) 383 [arXiv: 1506.06729] [INSPIRE].

[95] S. Whitsitt, W. Witczak-Krempa and S. Sachdev, Entanglement entropy of the large N Wilson-Fisher conformal field theory, Phys. Rev. B 95 (2017) 045148 [arXiv:1610.06568] [INSPIRE].

[96] T. Hirata and T. Takayanagi, AdS/CFT and strong subadditivity of entanglement entropy, JHEP 02 (2007) 042 [hep-th/0608213] [INSPIRE].

[97] M. Alishahiha, A.F. Astaneh, P. Fonda and F. Omidi, Entanglement Entropy for Singular Surfaces in Hyperscaling violating Theories, JHEP 09 (2015) 172 [arXiv:1507.05897] [INSPIRE].

[98] D.-W. Pang, Corner contributions to holographic entanglement entropy in non-conformal backgrounds, JHEP 09 (2015) 133 [arXiv:1506.07979] [INSPIRE].

[99] L. Bianchi, S. Chapman, X. Dong, D.A. Galante, M. Meineri and R.C. Myers, Shape dependence of holographic Rényi entropy in general dimensions, JHEP 11 (2016) 180 [arXiv: 1607.07418] [INSPIRE].

[100] M.R. Mohammadi Mozaffar, A. Mollabashi and F. Omidi, Holographic Mutual Information for Singular Surfaces, JHEP 12 (2015) 082 [arXiv:1511.00244] [INSPIRE].

[101] G. Pastras, On the Holographic Entanglement Entropy for Non-smooth Entangling Curves in $A d S_{4}$, Fortsch. Phys. 66 (2018) 1700090 [arXiv:1710.01948] [INSPIRE].

[102] M. Ghasemi and S. Parvizi, Entanglement entropy of singular surfaces under relevant deformations in holography, JHEP 02 (2018) 009 [arXiv: 1709.08169] [INSPIRE].

[103] E. Bakhshaei, A. Mollabashi and A. Shirzad, Holographic Subregion Complexity for Singular Surfaces, Eur. Phys. J. C $\mathbf{7 7}$ (2017) 665 [arXiv:1703.03469] [INSPIRE].

[104] E. Caceres and M.-L. Xiao, Complexity-action of subregions with corners, JHEP 03 (2019) 062 [arXiv: 1809.09356] [INSPIRE].

[105] M. Ghasemi and S. Parvizi, Relevant perturbation of entanglement entropy of singular surfaces, arXiv: 1902.05058 [INSPIRE].

[106] H. Dorn, On a new type of divergence for spiky Wilson loops and related entanglement entropies, JHEP 03 (2018) 124 [Erratum ibid. 05 (2018) 108] [arXiv:1801.10367] [INSPIRE].

[107] A.B. Kallin, M.B. Hastings, R.G. Melko and R.R.P. Singh, Anomalies in the entanglement properties of the square-lattice Heisenberg model, Phys. Rev. B 84 (2011) 165134 [arXiv: 1107.2840].

[108] A.B. Kallin, K. Hyatt, R.R.P. Singh and R.G. Melko, Entanglement at a Two-Dimensional Quantum Critical Point: A Numerical Linked-Cluster Expansion Study, Phys. Rev. Lett. 110 (2013) 135702 [arXiv: 1212.5269].

[109] S. Sahoo, E.M. Stoudenmire, J.-M. Stéphan, T. Devakul, R.R.P. Singh and R.G. Melko, Unusual Corrections to Scaling and Convergence of Universal Renyi Properties at Quantum Critical Points, Phys. Rev. B 93 (2016) 085120 [arXiv:1509.00468] [INSPIRE]. 
[110] A.B. Kallin, E.M. Stoudenmire, P. Fendley, R.R.P. Singh and R.G. Melko, Corner contribution to the entanglement entropy of an $O(3)$ quantum critical point in $2+1$ dimensions, J. Stat. Mech. 1406 (2014) P06009 [arXiv: 1401.3504] [InSPIRE].

[111] N. Laflorencie, D.J. Luitz and F. Alet, Spin-wave approach for entanglement entropies of the J1-J2 Heisenberg antiferromagnet on the square lattice, Phys. Rev. B 92 (2015) 115126 [arXiv:1506.03703] [INSPIRE].

[112] J. Helmes and S. Wessel, Correlations and entanglement in quantum critical bilayer and necklace XY models, Phys. Rev. B 92 (2015) 125120 [arXiv:1411.7773] [InSPIRE].

[113] C. De Nobili, A. Coser and E. Tonni, Entanglement negativity in a two dimensional harmonic lattice: Area law and corner contributions, J. Stat. Mech. 1608 (2016) 083102 [arXiv: 1604.02609] [INSPIRE].

[114] J. Helmes, L.E. Hayward Sierens, A. Chandran, W. Witczak-Krempa and R.G. Melko, Universal corner entanglement of Dirac fermions and gapless bosons from the continuum to the lattice, Phys. Rev. B 94 (2016) 125142 [arXiv: 1606.03096] [InSPIRE].

[115] T. Faulkner, R.G. Leigh and O. Parrikar, Shape Dependence of Entanglement Entropy in Conformal Field Theories, JHEP 04 (2016) 088 [arXiv:1511.05179] [INSPIRE].

[116] P. Bueno and W. Witczak-Krempa, Bounds on corner entanglement in quantum critical states, Phys. Rev. B 93 (2016) 045131 [arXiv:1511.04077] [INSPIRE].

[117] W. Witczak-Krempa, L.E. Hayward Sierens and R.G. Melko, Cornering gapless quantum states via their torus entanglement, Phys. Rev. Lett. 118 (2017) 077202 [arXiv: 1603.02684] [INSPIRE].

[118] C.-S. Chu and R.-X. Miao, Universality in the shape dependence of holographic Rényi entropy for general higher derivative gravity, JHEP 12 (2016) 036 [arXiv: 1608.00328] [INSPIRE].

[119] P. Bueno and R.C. Myers, Universal entanglement for higher dimensional cones, JHEP 12 (2015) 168 [arXiv: 1508.00587] [INSPIRE].

[120] B. Sirois, L.M. Fournier, J. Leduc and W. Witczak-Krempa, Geometric entanglement in integer quantum Hall states, Phys. Rev. B 103 (2021) 115115 [arXiv:2009.02337] [INSPIRE].

[121] H. Casini and M. Huerta, Positivity, entanglement entropy, and minimal surfaces, JHEP 11 (2012) 087 [arXiv: 1203.4007] [INSPIRE].

[122] E. Fradkin and J.E. Moore, Entanglement entropy of $2 D$ conformal quantum critical points: hearing the shape of a quantum drum, Phys. Rev. Lett. 97 (2006) 050404 [cond-mat/0605683] [INSPIRE].

[123] H. Casini, C.D. Fosco and M. Huerta, Entanglement and alpha entropies for a massive Dirac field in two dimensions, J. Stat. Mech. 0507 (2005) P07007 [cond-mat/0505563] [INSPIRE].

[124] H. Casini and M. Huerta, Remarks on the entanglement entropy for disconnected regions, JHEP 03 (2009) 048 [arXiv:0812.1773] [INSPIRE].

[125] B. Swingle, Mutual information and the structure of entanglement in quantum field theory, arXiv: 1010.4038 [INSPIRE].

[126] N. Drukker, D.J. Gross and H. Ooguri, Wilson loops and minimal surfaces, Phys. Rev. D 60 (1999) 125006 [hep-th/9904191] [INSPIRE]. 
[127] P. Fonda, L. Giomi, A. Salvio and E. Tonni, On shape dependence of holographic mutual information in $A d S_{4}, J H E P 02$ (2015) 005 [arXiv: 1411.3608] [INSPIRE].

[128] D.M. Hofman and J. Maldacena, Conformal collider physics: Energy and charge correlations, JHEP 05 (2008) 012 [arXiv:0803.1467] [INSPIRE].

[129] Y.-Z. Li, Holographic Studies of The Generic Massless Cubic Gravities, Phys. Rev. D 99 (2019) 066014 [arXiv:1901.03349] [INSPIRE].

[130] A.C. Wall, A Second Law for Higher Curvature Gravity, Int. J. Mod. Phys. D 24 (2015) 1544014 [arXiv: 1504.08040] [INSPIRE].

[131] S. Bhattacharyya, F.M. Haehl, N. Kundu, R. Loganayagam and M. Rangamani, Towards a second law for Lovelock theories, JHEP 03 (2017) 065 [arXiv:1612.04024] [INSPIRE]. 